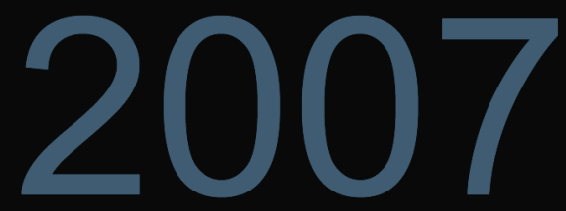

Lawrence Livermore National Laboratory Annual Illness and Injury Surveillance Report

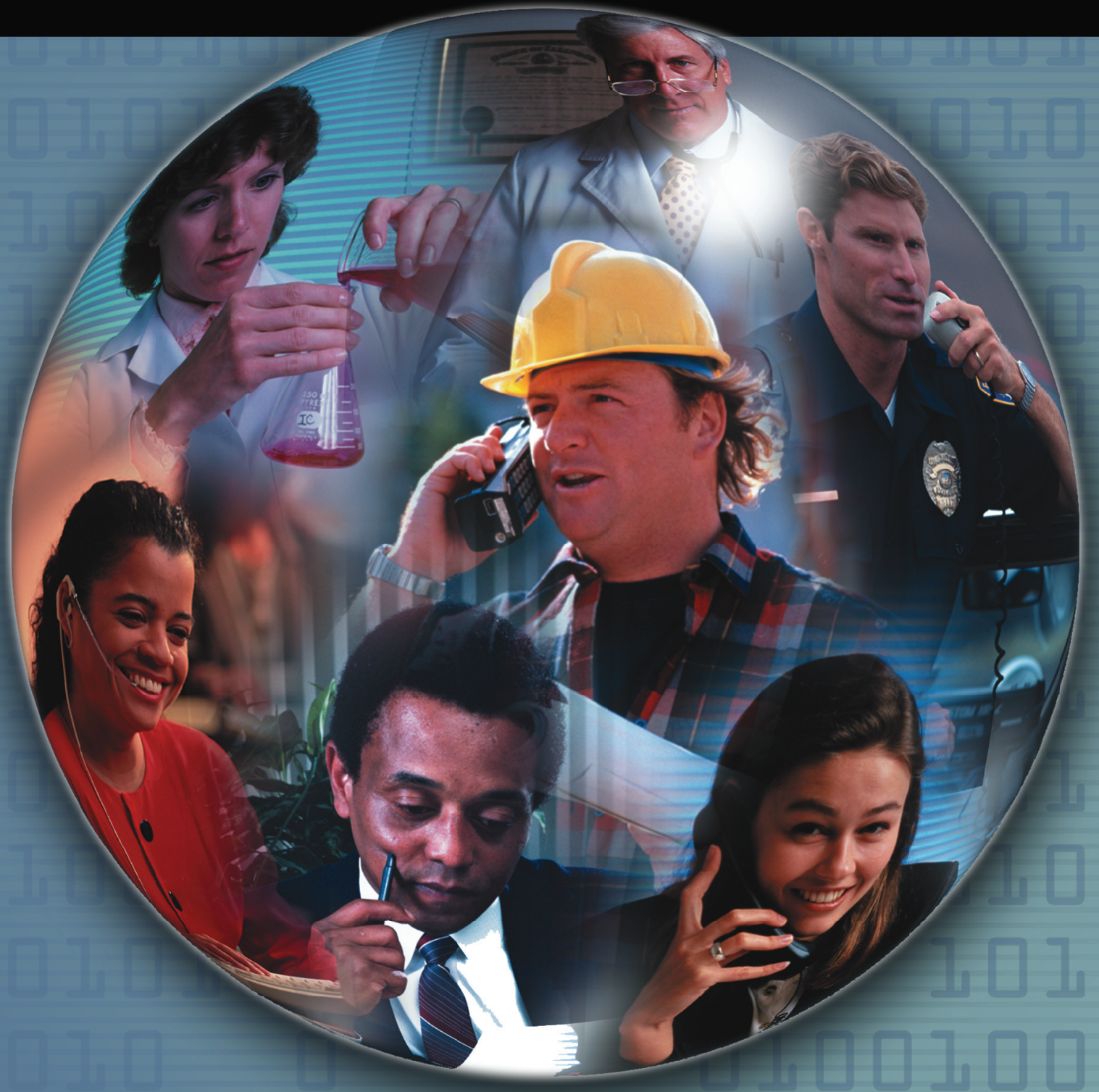




\section{Lawrence Livermore National Laboratory 2007 Illness and Injury Surveillance Report}

Questions or comments about this report or the Illness and Injury Surveillance Program (IISP) may be directed to:

E-mail:

Dr. Cliff Strader at cliff.strader@hq.doe.gov

or Dr. Bonnie Richter at bonnie.richter@hq.doe.gov

or direct letters to:

Mail Stop HS-13 / 270CC

U.S. Department of Energy

1000 Independence Avenue, S.W.

Washington, DC 20585-0270

Additional information about the Department of Energy's Office of Illness and Injury Prevention Programs, the IISP, and annual reports for DOE sites participating in this program can be found at:

http://www.hss.energy.gov/healthsafety/WSHP/epi/surv/

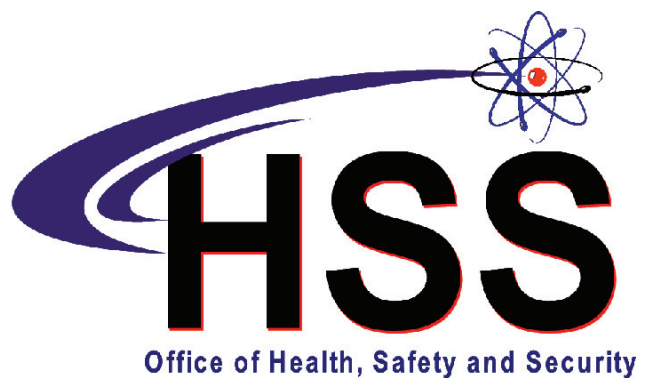

ACKNOWLEDGEMENT

LifeART images copyright 2000 Lippincott Williams \& Wilkins. All rights reserved.

This document was produced under contract number DE-AC05-06OR23100 between the

U.S. Department of Energy and Oak Ridge Associated Universities. 


\title{
Lawrence Livermore National Laboratory 2007 Illness and Injury Surveillance Report
}

\author{
At A Glance
}

A total of 8,570 LLNL employees were included in illness and injury surveillance in 2007; 2,657 (31 percent) women and 5,913 (69 percent) men.

The 221 absences among 198 women resulted in an absence rate of 8.3 per 100 workers $(221 / 2,657)$. The 377 absences reported by 347 men resulted in an absence rate of 6.4 per 100 workers $(377 / 5,913)$. Absence rates in 2007 were unchanged from those reported in 2006 for both men and women.

The average length of absences in 2007 was 24 days among men and 36 days among women. After experiencing a decrease in absences from 2004 to 2006, the average length of absences for women saw an increase in 2007, while among men the average length of absences has remained stable since 2003.

Among men, Service workers had the highest absence rate, 14.2 per 100 workers. Among women, Security and Fire workers had the highest absence rate, 20.0 per 100 workers.

Women were absent 7,947 calendar days due to illness and injury. Injuries (17.3 percent), respiratory disorders (14.5 percent), and musculoskeletal disorders (13.7 percent) accounted for 45.5 percent of all reported diagnoses among women.

Men lost 8,868 calendar days due to illness and injury. Respiratory conditions (18.8 percent), injuries (17.2 percent), and musculoskeletal disorders (14.6 percent accounted for 50.6 percent of all reported diagnoses among men.

Since 2003, respiratory and musculoskeletal conditions have been among the top 3 diagnostic categories reported by women, and injuries among the top 3 categories for men. This pattern is consistent with the overall observation of the top 3 diagnostic categories reported among sites participating in the Illness and Injury Surveillance Program (IISP) between 1995 and 2004.

LLNL reported 59 OSHA-recordable events among women and 99 OSHA-recordable events among men. The rate of workers with an OSHA event was similar for women and men $(2.2$ and 1.7 per 100 workers, respectively). Since 2003, the rates have held steady or declined, a pattern similar to what we have seen across the DOE Complex, where OSHA rates have been declining since 1995. 
Among women, the OSHA-recordable rates were highest among Crafts workers and among men highest among Security and Fire workers. These groups also had the highest OSHArecordable rates in 2006. OSHA-recordable rates were highest among men and women aged 30-39.

Eighty-six percent of the OSHA health conditions involved musculoskeletal conditions or injuries. This pattern was similar across all age groups.

Of the 145 OSHA events for which the type of accident was reported, 47.4 percent of the events for women were the result of repetitive trauma; among men, 44.3 percent were due to overexertion and strenuous movements. The conditions resulting from repetitive trauma reported by women included mainly joint pain and injuries. Only 3 carpal tunnel diagnoses were reported in this group; all were among women in the Administrative Support job category. 
The Lawrence Livermore National

Laboratory Work Force - 2007

The Work Force by Gender and Age

The Work Force by Gender and Job

Category ......................................... 1

\section{Number and Length of Absences}

Absence Rate by Gender and Age .2

Number of Days Absent by

Gender and Age

Absence Rate by Job Category

and Gender

Average Duration of Absence by

Job Category and Gender.

\section{Diagnostic Categories}

Number of Diagnoses and Lost Calendar

Days by Diagnostic Category

(Categorized by ICD-9-CM) and Gender......4

Common Diagnoses Among Female

Workers in 2007 .5

Common Diagnoses Among Male

Workers in 2007

Number of Most Frequently Reported

Diagnoses by Job Category and Gender ......7

\section{Rates of Disease Occurrence}

Rates for All Illnesses and Injuries

Combined by Job Category, Gender,

and Age

Rates for Selected Diagnostic Categories

by Job Category, Gender, and Age. ..8

\section{Time Trends}

Age-Adjusted Rates for All Diagnoses

Combined Among Women and Men

from 2003 to 2007
Age-Adjusted Rates for Selected Diagnostic Categories Among Women and Men from

2003 to 2007

Age-Adjusted Rates for All Diagnoses

Combined Among Women and Men by

Job Category from 2003 to 2007 .... 12

\section{Sentinel Health Events for Occupations (SHEOs)}

Characteristics of SHEOs by Gender. 13

SHEO Diagnoses by Gender 13

Occupational Safety and Health Administration (OSHA)-Recordable Events

OSHA-Recordable Events by Gender and Age 14

OSHA-Recordable Events by Job

Category and Gender 14

Diagnostic and Accident Categories for OSHA-Recordable Events

OSHA-Recordable Diagnoses by

Diagnostic Category and Gender .... 15

OSHA-Recordable Accidents by Type and Gender. 16

\section{Rates of OSHA-Recordable Events}

OSHA-Recordable Rates by Age and Job Categories Among Women, All

Diagnoses Combined 17

OSHA-Recordable Rates by Age and

Job Categories Among Men, All

Diagnoses Combined 17

Time Trends for OSHA-Recordable Events

Age-Adjusted Rates for All OSHA-Recordable Diagnoses Combined Among Women and Men by Job Category from 2003 to 2007 ... 18

\section{Appendices}

Appendices A-W. 20 
The Lawrence Livermore National Laboratory Work Force - 2007

Figure 1. The Work Force by Gender and Age

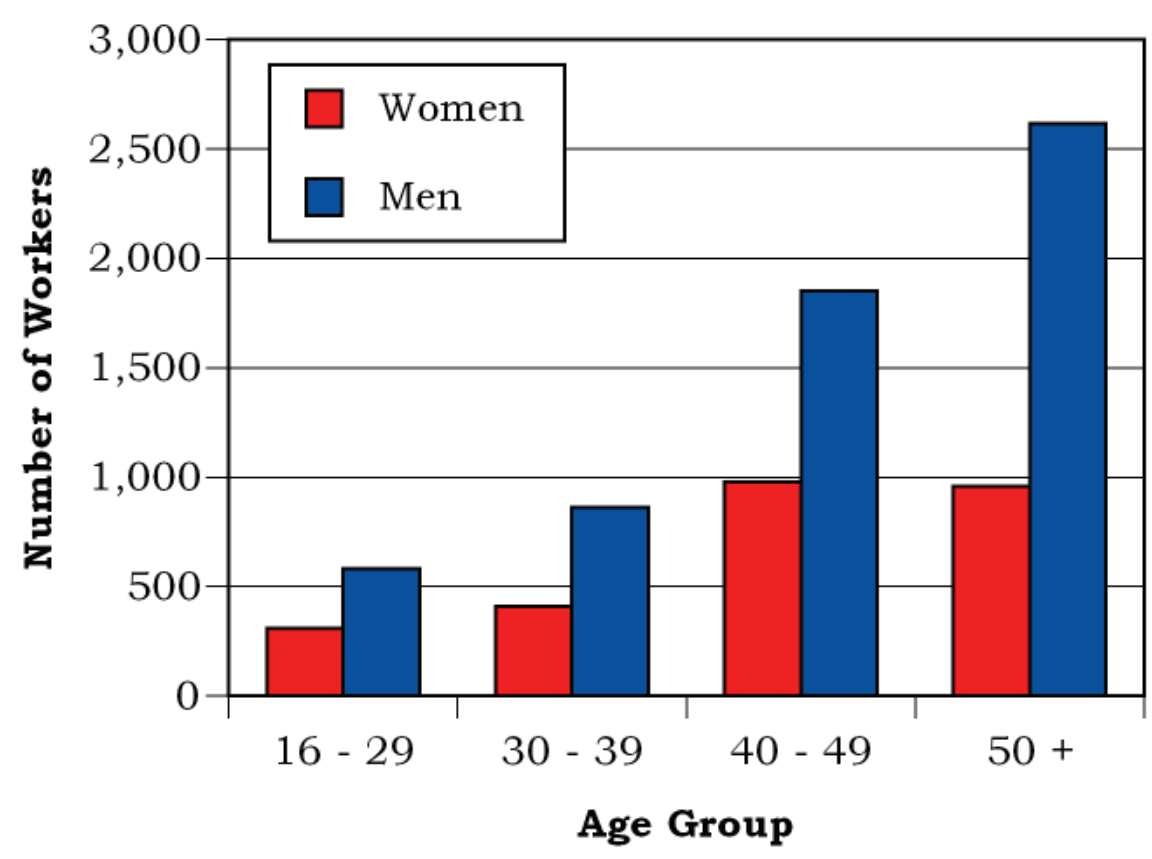

Figure 2. The Work Force by Gender and Job Category

\begin{tabular}{|l|c|c|}
\hline \multirow{2}{*}{ Job Category } & Women & Men \\
\hline \multirow{2}{*}{ Professional } & $\mathbf{5 8 8}$ & $\mathbf{2 , 6 9 6}$ \\
& $22 \%$ & $\mathbf{4 6} \%$ \\
\hline \multirow{2}{*}{ Administrative Support } & $\mathbf{1 , 7 0 4}$ & $\mathbf{1 , 7 1 2}$ \\
& $\mathbf{6 4 \%}$ & $\mathbf{2 9} \%$ \\
\hline \multirow{2}{*}{ Technical Support } & 231 & $\mathbf{6 7 1}$ \\
& $\mathbf{9} \%$ & $\mathbf{1 1} \%$ \\
\hline \multirow{2}{*}{ Service } & $\mathbf{8 9}$ & $\mathbf{2 2 6}$ \\
& $\mathbf{3} \%$ & $\mathbf{4} \%$ \\
\hline \multirow{2}{*}{ Security and Fire } & $\mathbf{3 5}$ & $\mathbf{2 8 9}$ \\
& $\mathbf{1} \%$ & $\mathbf{5} \%$ \\
\hline \multirow{2}{*}{ Crafts } & 10 & $\mathbf{3 1 9}$ \\
& $\mathbf{1} \%$ & $\mathbf{5} \%$ \\
\hline \multirow{2}{*}{ Line Operators } & $\mathbf{0}$ & $\mathbf{0}$ \\
& $\mathbf{0} \%$ & $\mathbf{0} \%$ \\
\hline \multirow{2}{*}{ Total } & $\mathbf{2 , 6 5 7}$ & $\mathbf{5 , 9 1 3}$ \\
\hline
\end{tabular}


Number and Length of Absences

Figure 3. Absence Rate by Gender and Age

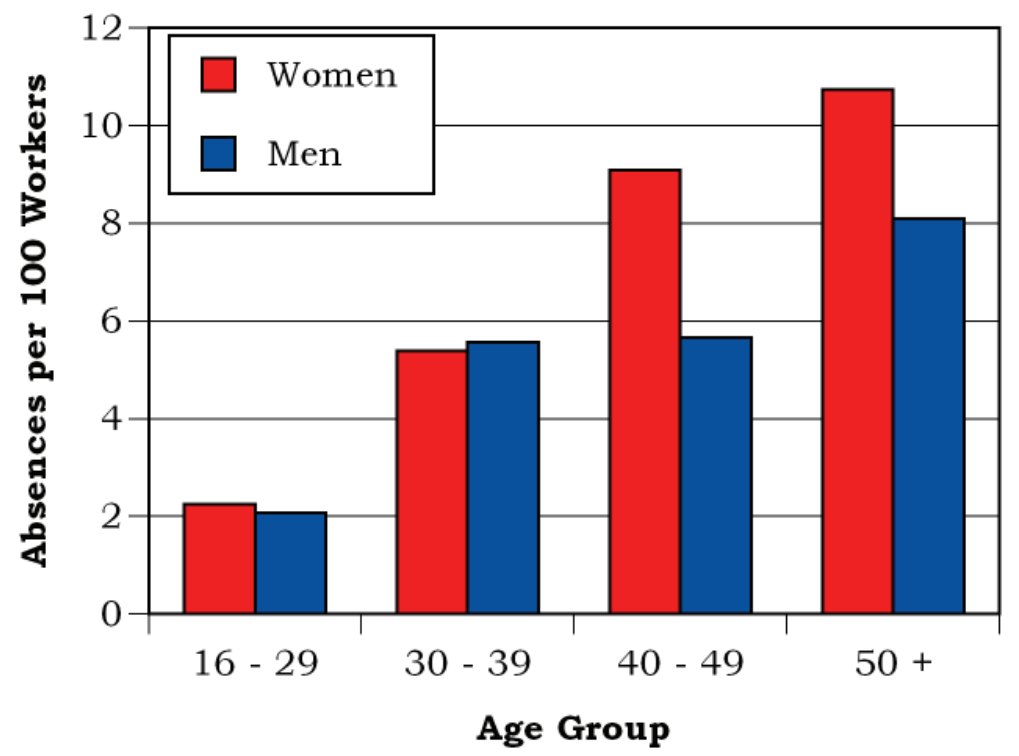

Figure 4. Number of Days Absent by Gender and Age

\begin{tabular}{|c|c|c|c|c|}
\hline \multirow{3}{*}{ Gender } & \multirow{2}{*}{ Age } & Number of & \multicolumn{2}{|c|}{ Number of Days Absent } \\
\cline { 3 - 5 } & & Absences & Total & Average \\
\hline \multirow{4}{*}{ Women } & $16-29$ & 7 & 95 & 14 \\
\cline { 2 - 5 } & $30-39$ & 22 & 928 & 42 \\
\cline { 2 - 5 } & $40-49$ & 89 & 3,232 & 36 \\
\cline { 2 - 5 } & $50+$ & 103 & 3,692 & 36 \\
\cline { 2 - 5 } & Total & 221 & 7,947 & 36 \\
\hline \multirow{4}{*}{ Men } & $16-29$ & 12 & 232 & 19 \\
\cline { 2 - 5 } & $30-39$ & 48 & 1,013 & 21 \\
\cline { 2 - 5 } & $40-49$ & 105 & 2,234 & 21 \\
\cline { 2 - 5 } & $50+$ & 212 & 5,389 & 25 \\
\cline { 2 - 5 } & Total & 377 & 8,868 & 24 \\
\hline
\end{tabular}


Figure 5. Absence Rate by Job Category and Gender

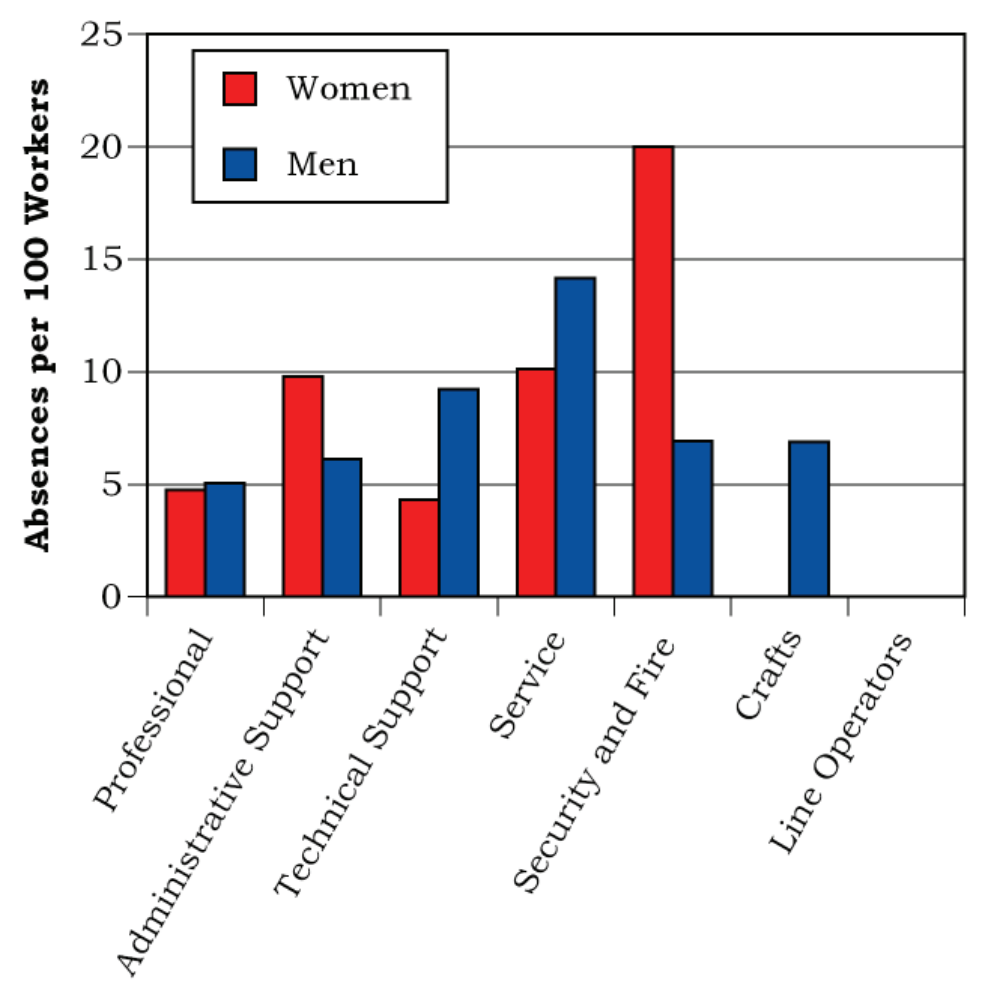

Job Category

Figure 6. Average Duration of Absence by Job Category and Gender

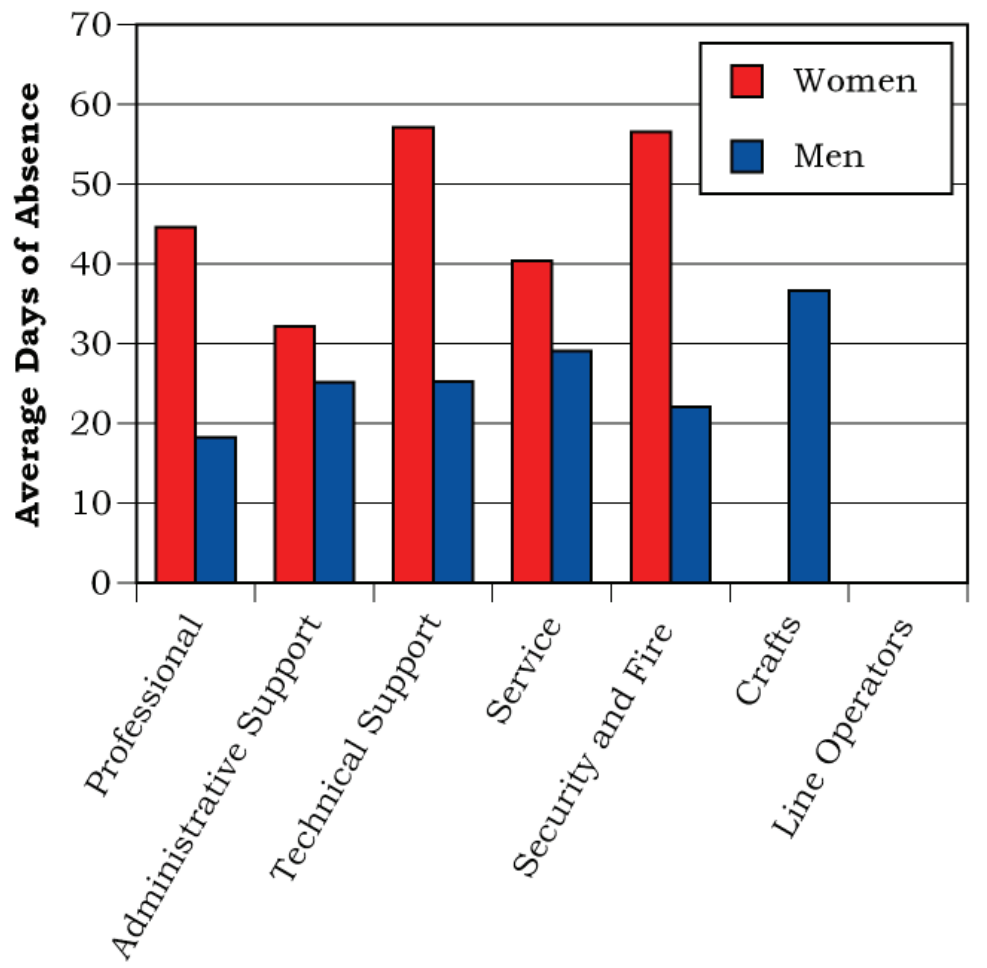




\section{Diagnostic Categories}

\section{Figure 7. Number of Diagnoses and Lost Calendar Days by Diagnostic Category (Categorized by ICD-9-CM) and Gender}

\begin{tabular}{|c|c|c|c|c|}
\hline \multirow[b]{2}{*}{ Diagnostic Category } & \multicolumn{2}{|c|}{ Women } & \multicolumn{2}{|c|}{ Men } \\
\hline & $\begin{array}{l}\text { Number of } \\
\text { Diagnoses }\end{array}$ & $\begin{array}{c}\text { Number } \\
\text { of Lost } \\
\text { Calendar } \\
\text { Days }\end{array}$ & $\begin{array}{l}\text { Number of } \\
\text { Diagnoses }\end{array}$ & $\begin{array}{c}\text { Number } \\
\text { of Lost } \\
\text { Calendar } \\
\text { Days }\end{array}$ \\
\hline Benign Growths & 13 & 600 & 7 & 195 \\
\hline Blood & 2 & 440 & 1 & 66 \\
\hline Cancer & 16 & 819 & 16 & 955 \\
\hline Digestive & 15 & 779 & 55 & 1,096 \\
\hline Endocrine/Metabolic & 3 & 132 & 6 & 196 \\
\hline Existing Birth Condition & 0 & 0 & 0 & 0 \\
\hline Genitourinary & 28 & 931 & 10 & 296 \\
\hline Heart/Circulatory & 7 & 199 & 26 & 930 \\
\hline Infections / Parasites & 9 & 49 & 35 & 711 \\
\hline Injury & 44 & 1,807 & 75 & 1,955 \\
\hline Miscarriage & 3 & 67 & NA & NA \\
\hline Musculoskeletal & 35 & 2,134 & 64 & 1,908 \\
\hline Nervous System & 18 & 199 & 23 & 666 \\
\hline Psychological & 5 & 454 & 3 & 83 \\
\hline Respiratory & 37 & 482 & 82 & 715 \\
\hline Skin & 3 & 77 & 6 & 211 \\
\hline Unspecified Symptoms & 17 & 381 & 28 & 385 \\
\hline
\end{tabular}

Note: Lost calendar days for each absence are counted more than once when multiple diagnoses occur in different diagnostic categories for the same absence. 
Figure 8. Common Diagnoses Among Female Workers in 2007

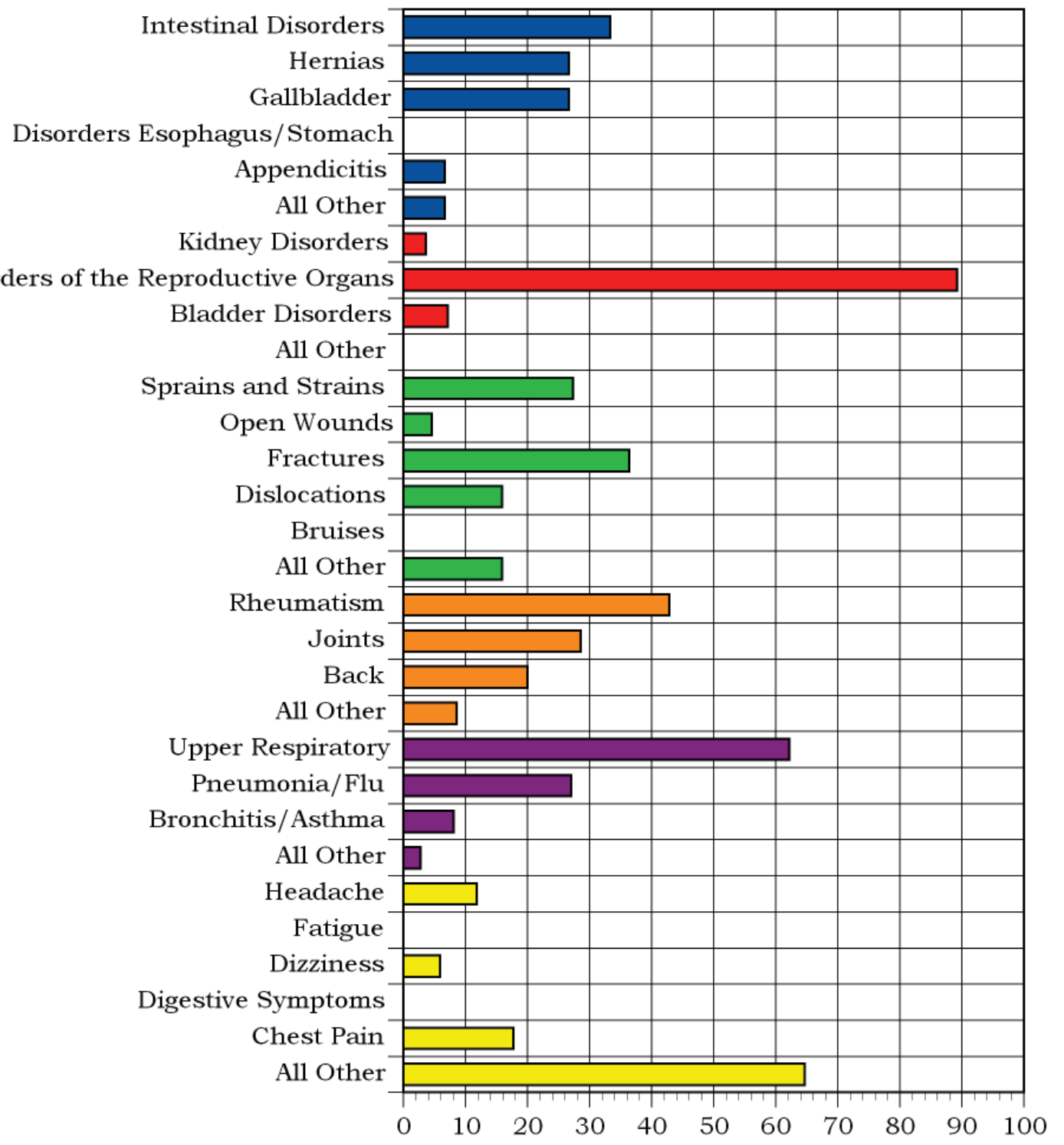

Percent Distribution of Diagnoses Within Diagnostic Category

Digestive, 15 Diagnoses

Genitourinary, 28 Diagnoses

Injury, 44 Diagnoses
Musculoskeletal, 35 Diagnoses

Respiratory, 37 Diagnoses

Unspecified Symptoms, 17 Diagnoses 
Figure 9. Common Diagnoses Among Male Workers in 2007

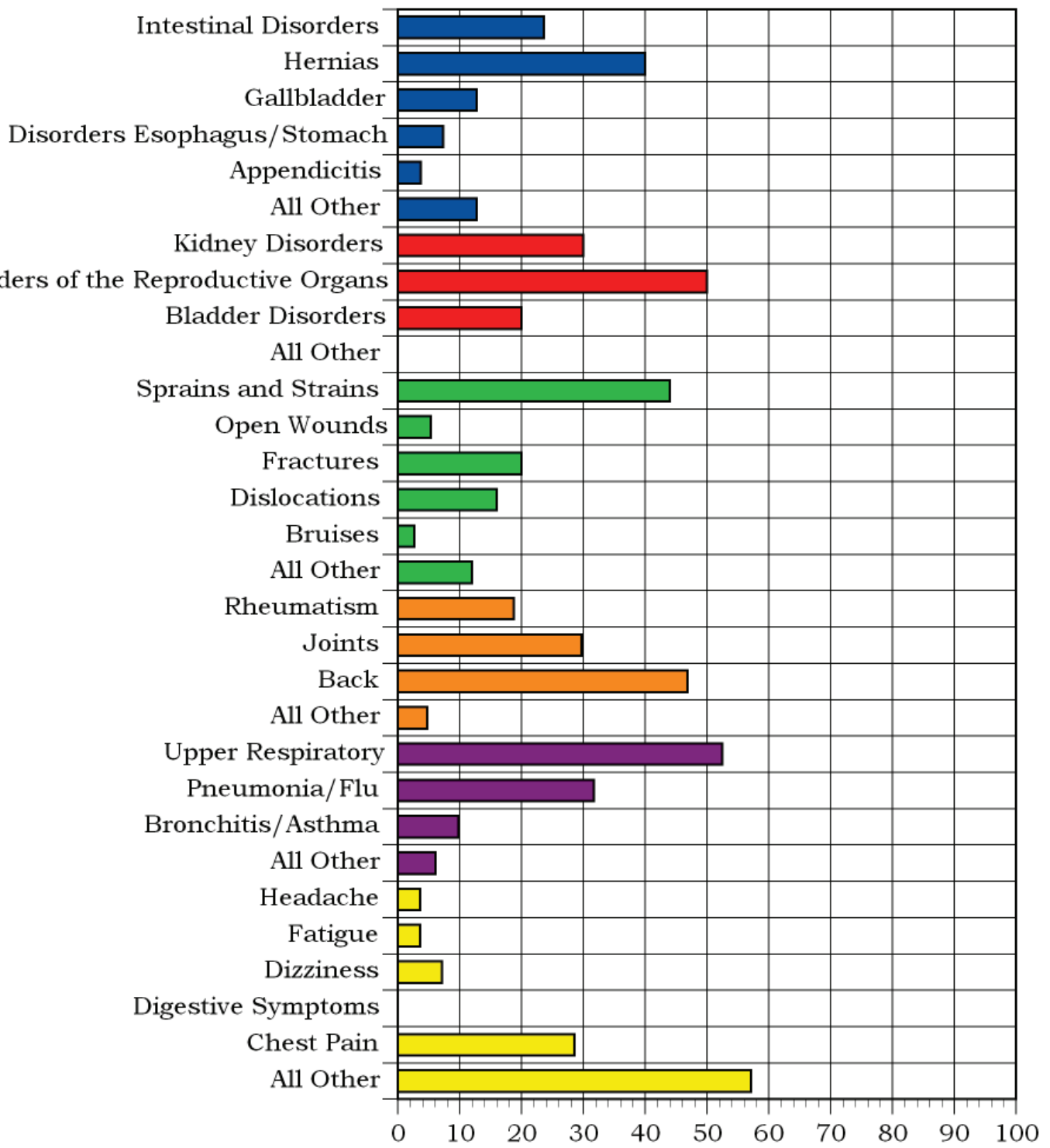

Percent Distribution of Diagnoses Within Diagnostic Category

\begin{tabular}{ll}
$\square$ Digestive, 55 Diagnoses & $\square$ Musculoskeletal, 64 Diagnoses \\
$\square$ Genitourinary, 10 Diagnoses & $\square$ Respiratory, 82 Diagnoses \\
$\square$ Injury, 75 Diagnoses & $\square$ Unspecified Symptoms, 28 Diagnoses \\
\hline
\end{tabular}




\section{Figure 10. Number of Most Frequently Reported} Diagnoses by Job Category and Gender

\begin{tabular}{|c|c|c|c|c|}
\hline Job Category & \multicolumn{2}{|l|}{ Men } & \multicolumn{2}{|l|}{ Women } \\
\hline \multirow{3}{*}{ Professional } & Respiratory & 30 & Respiratory & 7 \\
\hline & Musculoskeletal & 26 & Injury & 5 \\
\hline & Injury & 21 & Musculoskeletal & 5 \\
\hline \multirow{3}{*}{ Administrative Support } & Respiratory & 22 & Injury & 34 \\
\hline & Digestive & 15 & Genitourinary & 25 \\
\hline & Injury & 15 & Respiratory & 23 \\
\hline \multirow{3}{*}{ Technical Support } & Injury & 18 & Musculoskeletal & 3 \\
\hline & Digestive & 12 & Respiratory & 2 \\
\hline & Respiratory & 12 & Unspecified Symptoms & 2 \\
\hline \multirow{6}{*}{ Service } & Respiratory & 11 & Injury & 4 \\
\hline & Digestive & 8 & Respiratory & 3 \\
\hline & Injury & 7 & Benign Growths & 1 \\
\hline & & & Digestive & 1 \\
\hline & & & Musculoskeletal & 1 \\
\hline & & & Unspecified Symptoms & 1 \\
\hline \multirow{3}{*}{ Security and Fire } & Injury & 9 & Musculoskeletal & 4 \\
\hline & Musculoskeletal & 7 & Cancer & 2 \\
\hline & Respiratory & 4 & Respiratory & 2 \\
\hline \multirow{4}{*}{ Crafts } & Musculoskeletal & 7 & & 0 \\
\hline & Injury & 5 & & \\
\hline & Digestive & 3 & & \\
\hline & Respiratory & 3 & & \\
\hline Line Operators & & 0 & & 0 \\
\hline
\end{tabular}




\section{Rates of Disease Occurrence}

Figure 11. Rates for All Illnesses and Injuries Combined by Job Category, Gender, and Age

\begin{tabular}{|c|c|c|c|c|}
\hline \multirow{2}{*}{$\begin{array}{c}\text { All Illnesses \& } \\
\text { Injuries Combined }\end{array}$} & \multicolumn{4}{|c|}{ Rate per 1,000} \\
\hline & Job Category & Age & Men & Women \\
\hline & \multirow{2}{*}{ Professional } & $<50$ & 45 & 42 \\
\hline & & $50+$ & 73 & 81 \\
\hline & \multirow{2}{*}{ Administrative Support } & $<50$ & 52 & 94 \\
\hline & & $50+$ & 87 & 140 \\
\hline & \multirow{2}{*}{ Technical Support } & $<50$ & 77 & 26 \\
\hline & & $50+$ & 150 & 89 \\
\hline & \multirow{2}{*}{ Service } & $<50$ & 177 & 128 \\
\hline & & $50+$ & 177 & 119 \\
\hline & \multirow{2}{*}{ Security and Fire } & $<50$ & 82 & 267 \\
\hline & & $50+$ & 136 & 600 \\
\hline & \multirow{2}{*}{ Crafts } & $<50$ & 50 & 0 \\
\hline & & $50+$ & 121 & 0 \\
\hline$y$ & \multirow{2}{*}{ Line Operators } & $<50$ & 0 & 0 \\
\hline 10 & & $50+$ & 0 & 0 \\
\hline
\end{tabular}

Figure 12. Rates for Selected Diagnostic Categories by Job Category, Gender, and Age

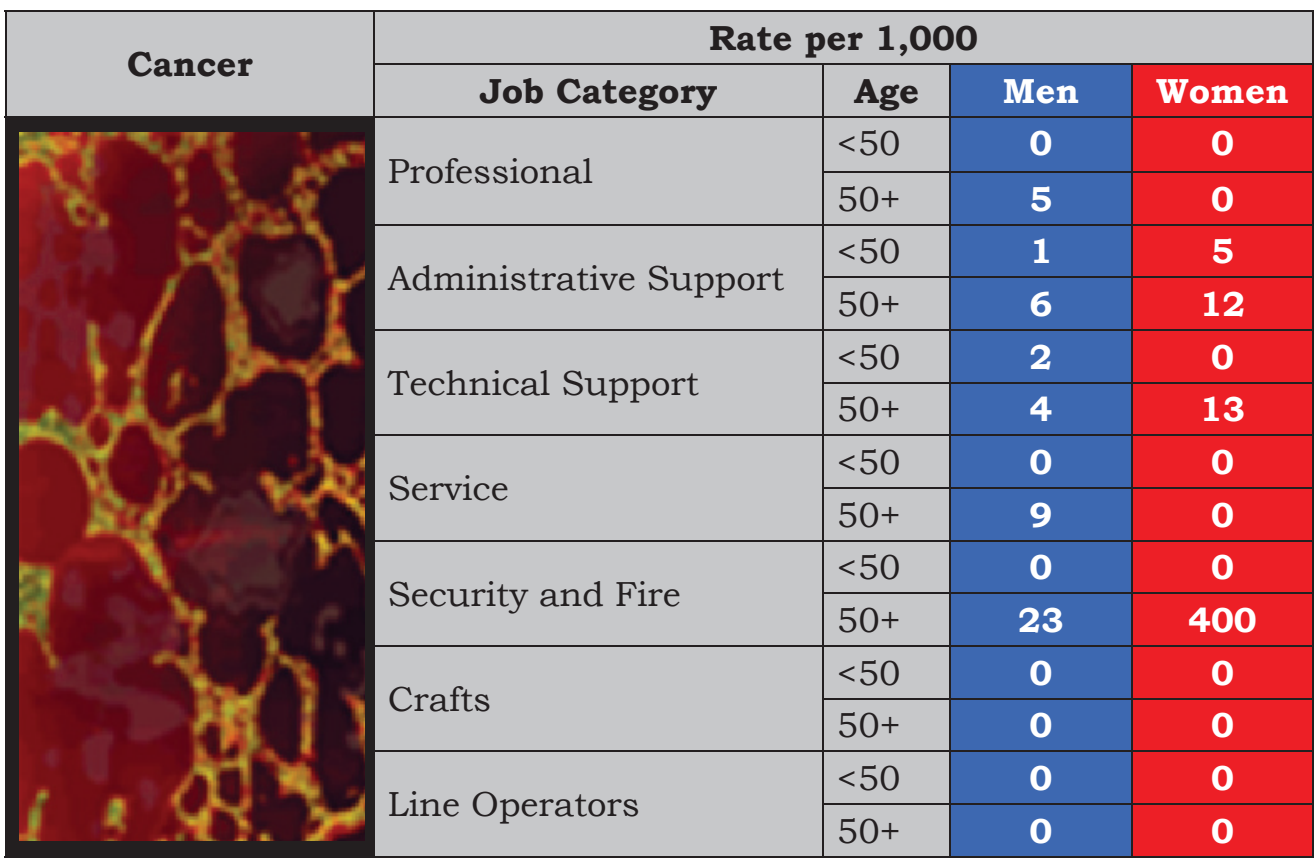




\section{Figure 12. Rates for Selected Diagnostic Categories by Job Category, Gender, and Age (Continued)}

\begin{tabular}{|c|c|c|c|c|}
\hline \multirow{2}{*}{ Heart/Circulatory } & \multicolumn{4}{|c|}{ Rate per 1,000} \\
\hline & Job Category & Age & Men & Women \\
\hline & \multirow{2}{*}{ Professional } & $<50$ & 1 & 0 \\
\hline & & $50+$ & 9 & 5 \\
\hline & \multirow{2}{*}{ Administrative Support } & $<50$ & 4 & 2 \\
\hline & & $50+$ & 5 & 5 \\
\hline & \multirow{2}{*}{ Technical Support } & $<50$ & 0 & 7 \\
\hline & & $50+$ & 7 & 0 \\
\hline & \multirow{2}{*}{ Service } & $<50$ & 9 & 0 \\
\hline & & $50+$ & 0 & 0 \\
\hline & \multirow{2}{*}{ Security and Fire } & $<50$ & 4 & 0 \\
\hline & & $50+$ & 0 & 0 \\
\hline & \multirow{2}{*}{ Crafts } & $<50$ & 0 & 0 \\
\hline & & $50+$ & 7 & 0 \\
\hline & \multirow{2}{*}{ Line Operators } & $<50$ & 0 & 0 \\
\hline & & $50+$ & 0 & 0 \\
\hline
\end{tabular}

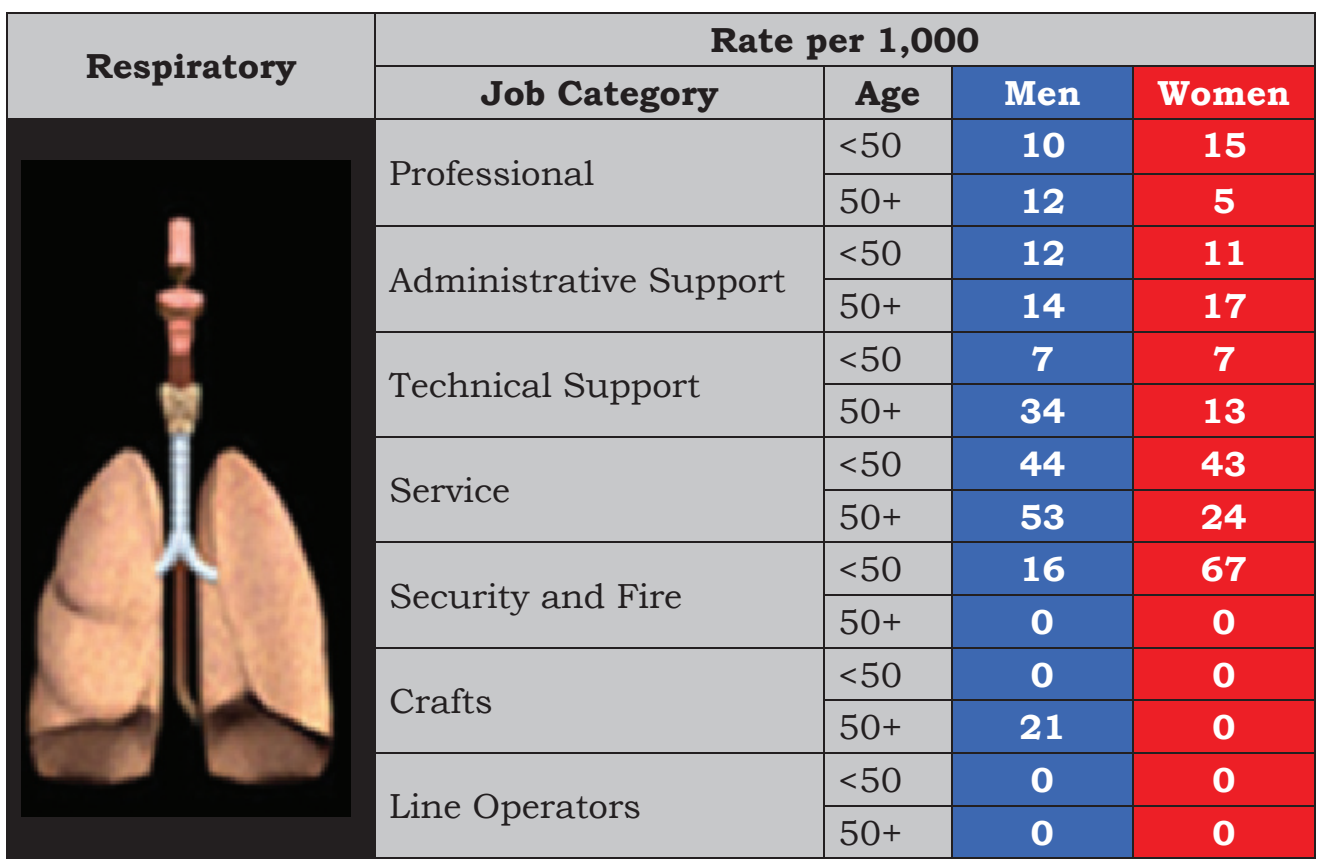


Figure 12. Rates for Selected Diagnostic Categories by Job Category, Gender, and Age (Continued)

\begin{tabular}{|c|c|c|c|c|}
\hline \multirow{2}{*}{ Injury } & \multicolumn{4}{|c|}{ Rate per 1,000} \\
\hline & Job Category & Age & Men & Women \\
\hline \multirow{14}{*}{1} & \multirow{2}{*}{ Professional } & $<50$ & 8 & 2 \\
\hline & & $50+$ & 8 & 22 \\
\hline & \multirow{2}{*}{ Administrative Support } & $<50$ & 6 & 17 \\
\hline & & $50+$ & 12 & 25 \\
\hline & \multirow{2}{*}{ Technical Support } & $<50$ & 30 & 0 \\
\hline & & $50+$ & 22 & 0 \\
\hline & \multirow{2}{*}{ Service } & $<50$ & 27 & 64 \\
\hline & & $50+$ & 35 & 24 \\
\hline & \multirow{2}{*}{ Security and Fire } & $<50$ & 20 & 33 \\
\hline & & $50+$ & 91 & 0 \\
\hline & \multirow{2}{*}{ Crafts } & $<50$ & 6 & 0 \\
\hline & & $50+$ & 29 & 0 \\
\hline & \multirow{2}{*}{ Line Operators } & $<50$ & 0 & 0 \\
\hline & & $50+$ & 0 & 0 \\
\hline
\end{tabular}

Time Trends

Figure 13. Age-Adjusted Rates for All Diagnoses Combined Among Women and Men from 2003 to 2007*

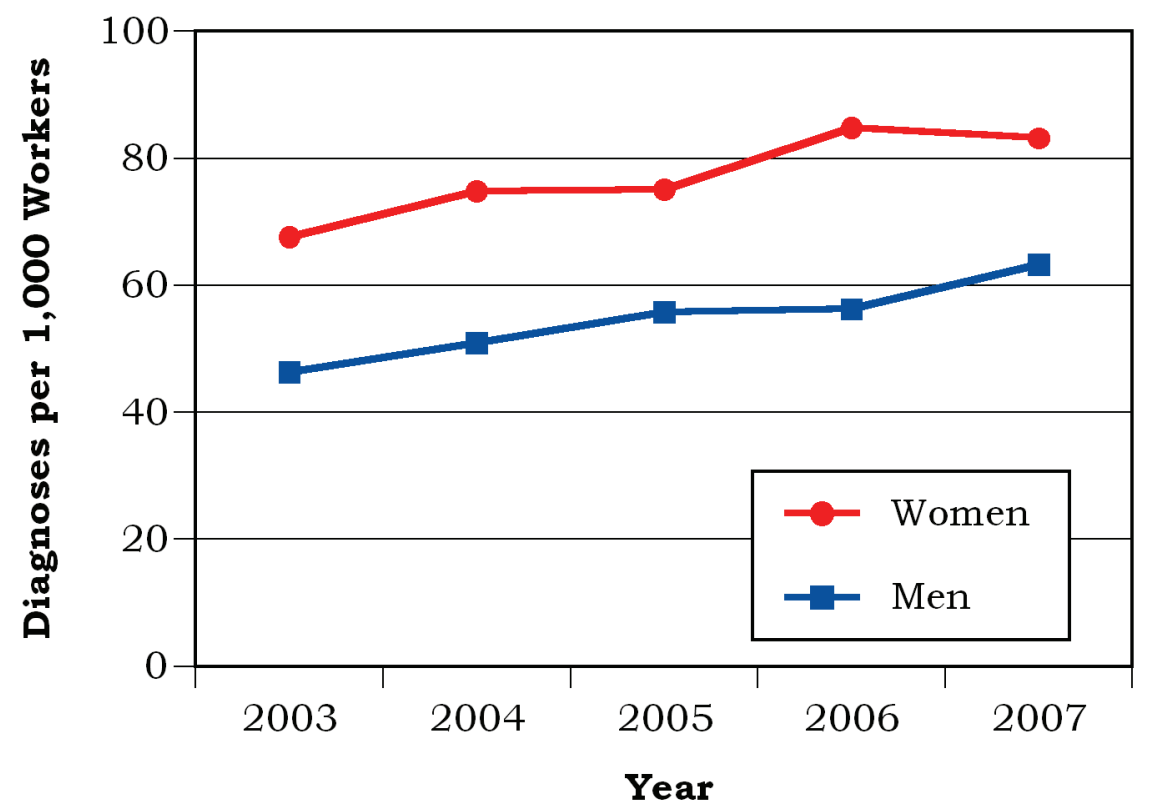

* Standardized to age distribution of 2000 U.S. population. 
Figure 14. Age-Adjusted Rates for Selected Diagnostic

Categories Among Women and Men from 2003 to 2007*

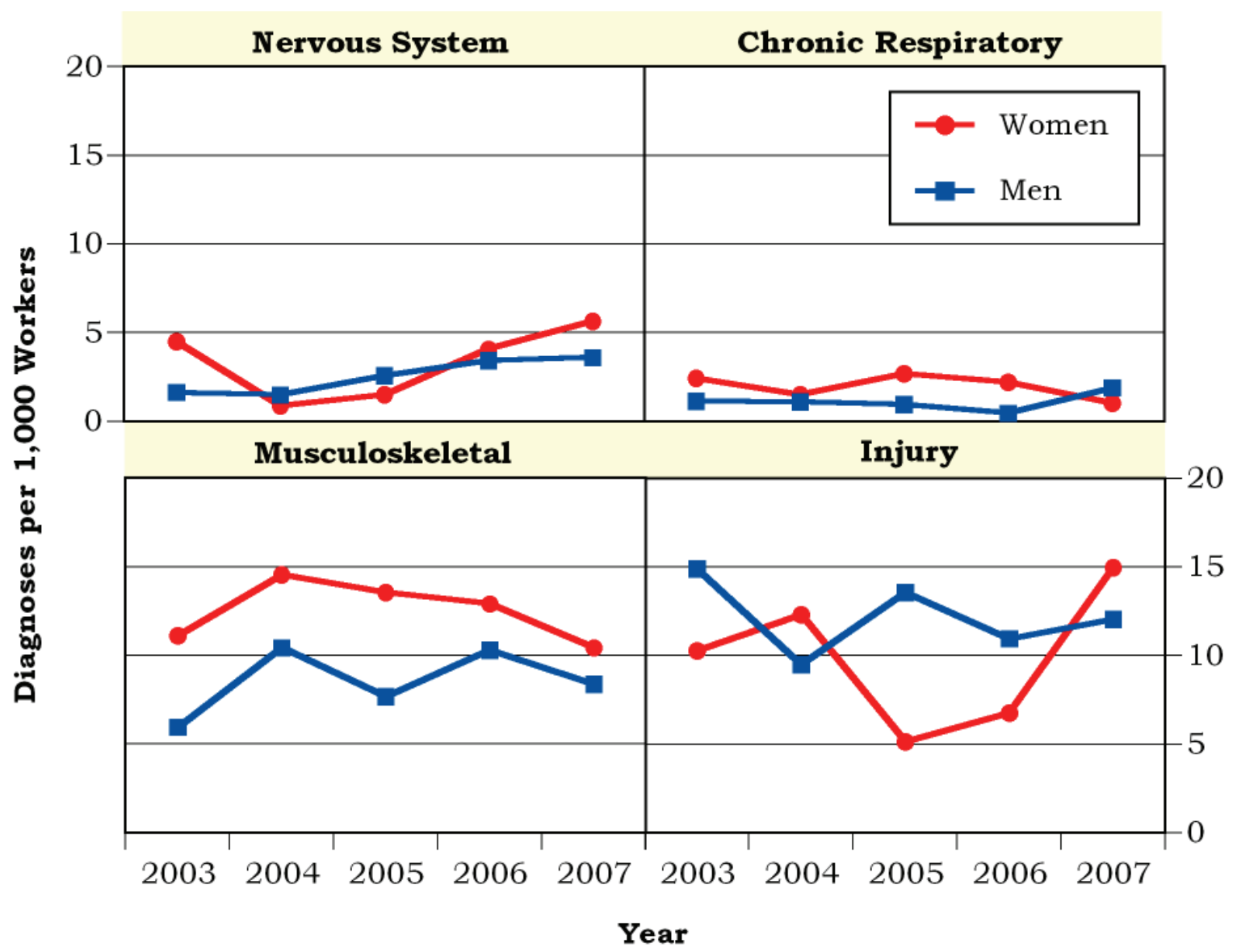

* Standardized to age distribution of 2000 U.S. population. 
Figure 15. Age-Adjusted Rates for All Diagnoses Combined Among Women and Men by Job Category from 2003 to 2007*
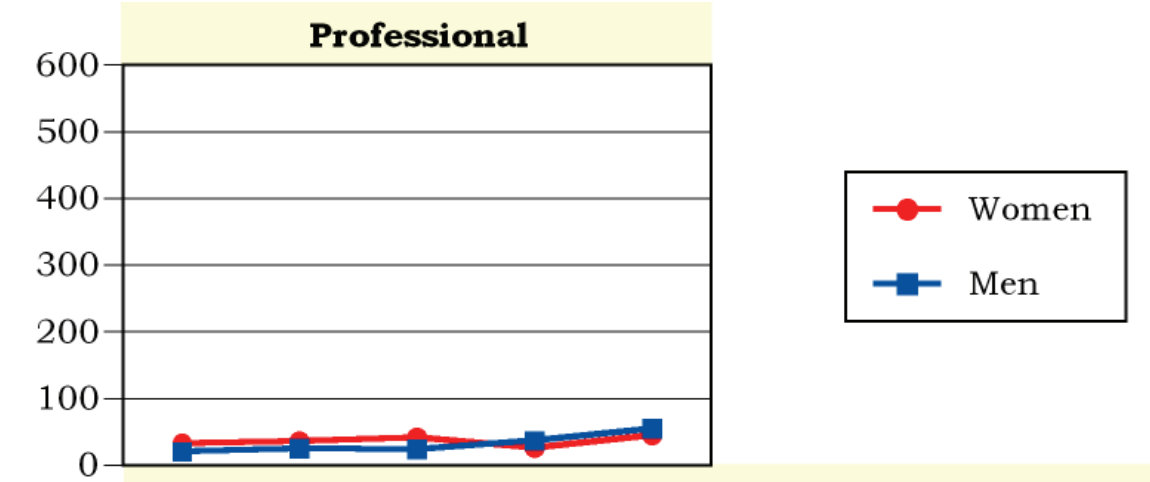

\section{Technical Support}

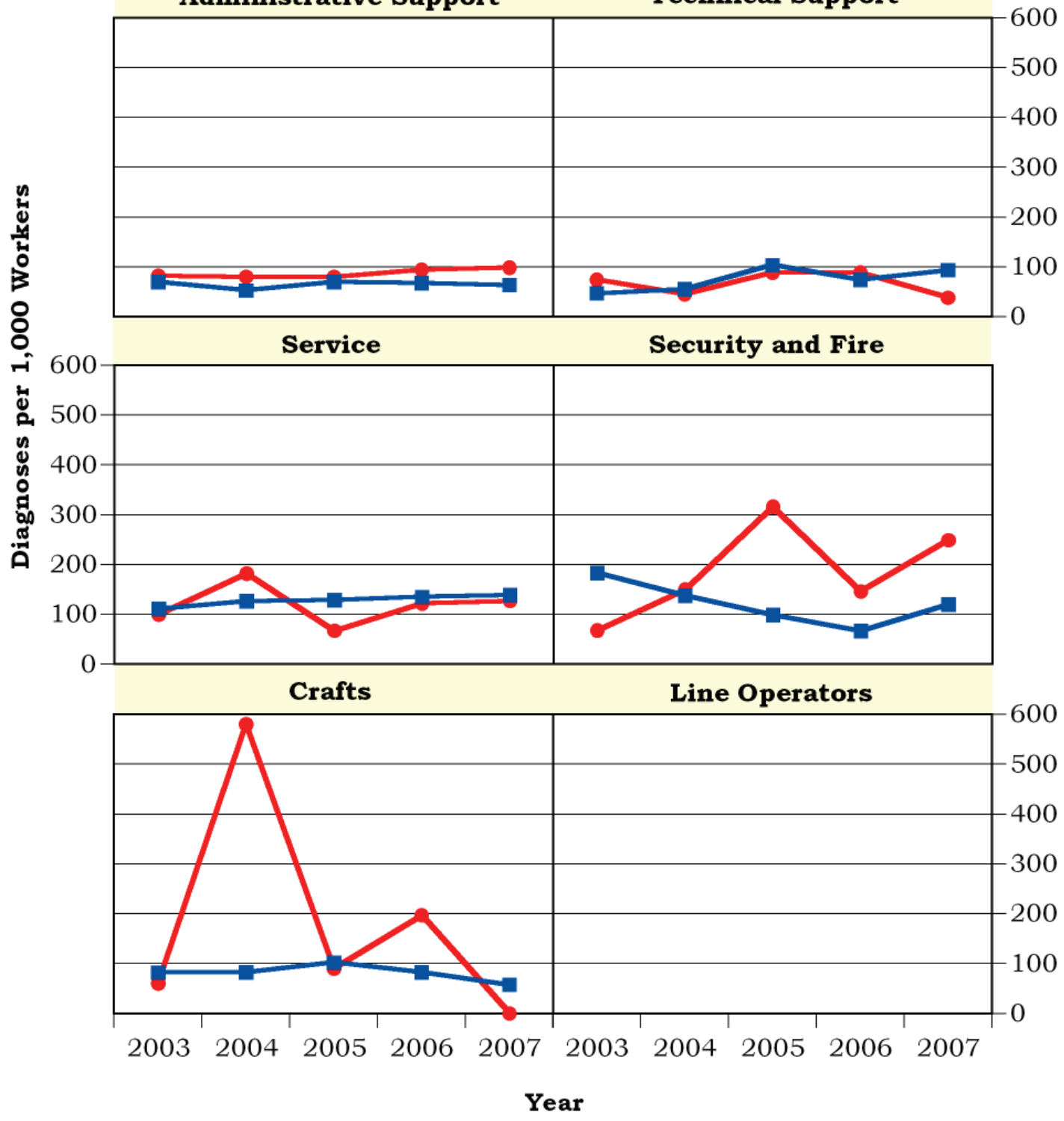

* Standardized to age distribution of 2000 U.S. population. 


\section{Sentinel Health Events for Occupations (SHEOs)}

An occupational sentinel health event (SHEO) is a disease, disability, or death that is likely to be occupationally related. Although sentinel health events may indicate an occupational exposure, many may result from nonoccupational exposures. Sentinel health events are therefore assessed in two categories:

Definite Sentinel Health Events: Diseases that are unlikely to occur in the absence of an occupational exposure (e.g., asbestosis).

Possible Sentinel Health Events: Diseases that may be occupational but can also occur in the absence of an occupational exposure (e.g., lung cancer or carpal tunnel syndrome).

Figure 16. Characteristics of SHEOs by Gender

\begin{tabular}{|l|c|c|c|c|}
\hline \multirow{2}{*}{} & \multicolumn{2}{|c|}{$\begin{array}{c}\text { Total Number of } \\
\text { SHEO Diagnoses }\end{array}$} & \multicolumn{2}{c|}{$\begin{array}{c}\text { Total Number of } \\
\text { Days Absent }\end{array}$} \\
\cline { 2 - 5 } & Men & Women & Men & Women \\
\hline Definite & 1 & 0 & 54 & 0 \\
\hline Possible & 5 & 3 & 279 & 24 \\
\hline Total & 6 & 3 & 333 & 24 \\
\hline
\end{tabular}

Figure 17. SHEO Diagnoses by Gender

\begin{tabular}{|l|c|c|}
\hline \multirow{2}{*}{\multicolumn{1}{|c|}{ Diagnoses }} & \multicolumn{2}{c|}{ Gender } \\
\cline { 2 - 3 } & Women & Men \\
\hline Carpal Tunnel Syndrome & 3 & 3 \\
\hline Pneumonconiosis (Berylliosis) & 0 & 0 \\
\hline Musculoskeletal Conditions & 0 & 1 \\
\hline Injuries & 0 & 0 \\
\hline Other Conditions & 0 & 2 \\
\hline
\end{tabular}


Occupational Safety and Health Administration (OSHA)-Recordable Events Figure 18. OSHA-Recordable Events by Gender and Age

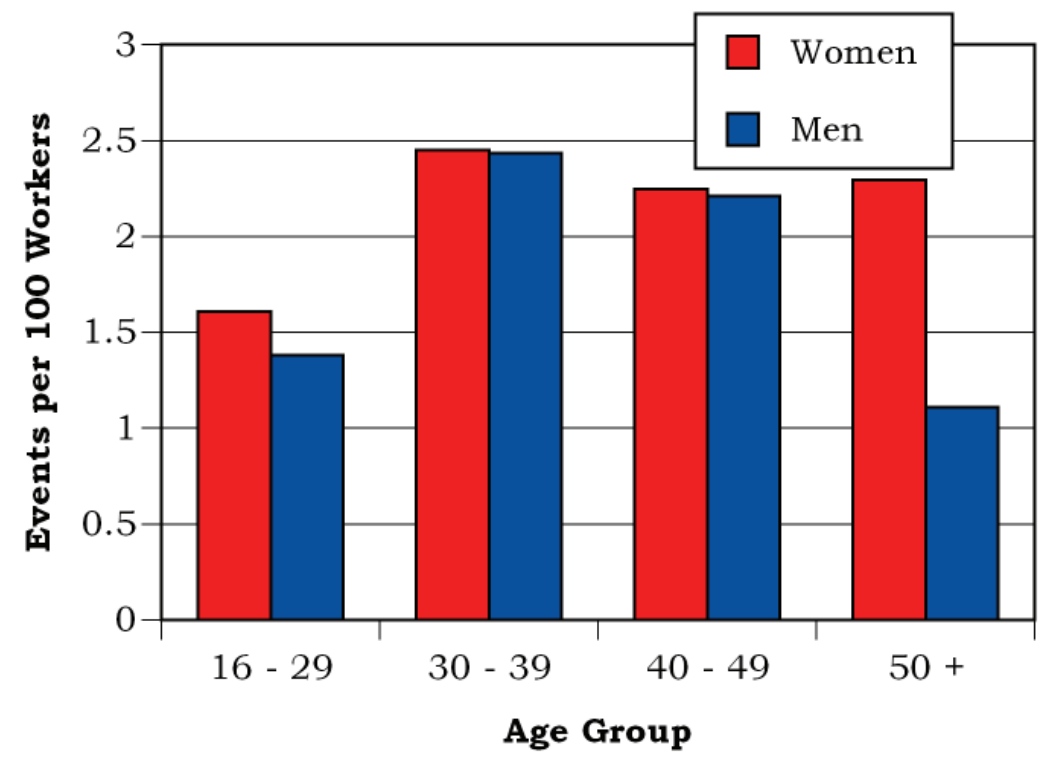

Figure 19. OSHA-Recordable Events by Job Category and Gender

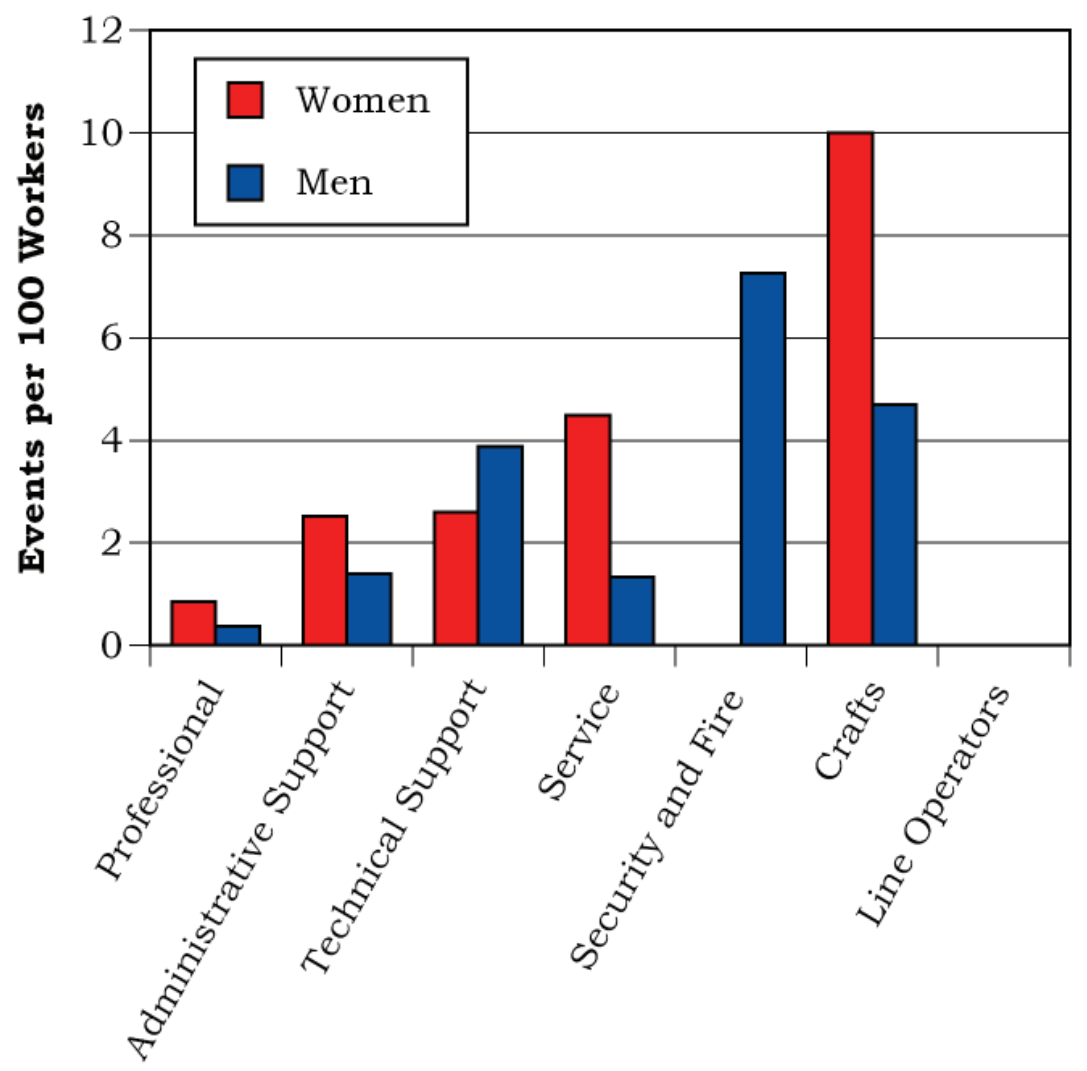

Job Category 


\section{Diagnostic and Accident Categories for OSHA-Recordable Events}

Figure 20. OSHA-Recordable Diagnoses by Diagnostic Category and Gender

\begin{tabular}{|l|c|c|}
\hline \multirow{2}{*}{\multicolumn{1}{|c|}{ Diagnostic Category }} & \multicolumn{2}{|c|}{ Gender } \\
\cline { 2 - 3 } Blood & Women & Men \\
\hline Digestive & $\mathbf{0}$ & $\mathbf{1}$ \\
\hline Infections / Parasites & $\mathbf{1}$ & $\mathbf{1}$ \\
\hline Musculoskeletal & $\mathbf{4 1}$ & $\mathbf{6 4}$ \\
\hline Nervous System & $\mathbf{4}$ & $\mathbf{4}$ \\
\hline Respiratory & $\mathbf{1}$ & $\mathbf{1}$ \\
\hline Skin & $\mathbf{2}$ & $\mathbf{2}$ \\
\hline Unspecified Symptoms & $\mathbf{9}$ & $\mathbf{1 3}$ \\
\hline Injury & $\mathbf{5 4}$ & $\mathbf{9 2}$ \\
\hline Fractures - Upper Limb & $\mathbf{1}$ & $\mathbf{3}$ \\
\hline Fractures - Lower Limb & $\mathbf{0}$ & $\mathbf{1}$ \\
\hline Dislocations & $\mathbf{0}$ & $\mathbf{3}$ \\
\hline Back Sprains \& Strains & $\mathbf{4}$ & $\mathbf{1 2}$ \\
\hline Other Sprains \& Strains & $\mathbf{1 5}$ & $\mathbf{2 8}$ \\
\hline Open Wounds - Head, Neck, Trunk & $\mathbf{0}$ & $\mathbf{2}$ \\
\hline Open Wounds - Upper Limb & $\mathbf{0}$ & $\mathbf{9}$ \\
\hline Open Wounds - Lower Limb & $\mathbf{0}$ & $\mathbf{1}$ \\
\hline Superficial Injuries & $\mathbf{2}$ & $\mathbf{1}$ \\
\hline Bruises & $\mathbf{5}$ & $\mathbf{8}$ \\
\hline Foreign Bodies Entering Orifice & $\mathbf{0}$ & $\mathbf{2}$ \\
\hline Burns & $\mathbf{0}$ & $\mathbf{1}$ \\
\hline Unspecified Injuries & $\mathbf{2 5}$ & $\mathbf{1 9}$ \\
\hline Adverse Reactions to Non-Medical & $\mathbf{0}$ & $\mathbf{1}$ \\
\hline Substances & & $\mathbf{1}$ \\
\hline Adverse Reactions to External Causes & & \\
\hline
\end{tabular}


Figure 21. OSHA-Recordable Accidents by Type and Gender

\begin{tabular}{|l|c|c|}
\hline \multirow{2}{*}{\multicolumn{1}{|}{ Accident Category }} & \multicolumn{2}{c|}{ Gender } \\
\cline { 2 - 3 } & Women & Men \\
\cline { 2 - 3 } & $\begin{array}{c}\text { Number of } \\
\text { Accidents }\end{array}$ & $\begin{array}{c}\text { Number of } \\
\text { Accidents }\end{array}$ \\
\hline Motor Vehicle Traffic & $\mathbf{1}$ & $\mathbf{0}$ \\
\hline Non-Motor Vehicle & $\mathbf{0}$ & $\mathbf{2}$ \\
\hline Poisoning-Non-Medicinal & $\mathbf{1}$ & $\mathbf{0}$ \\
\hline Falls & $\mathbf{1 1}$ & $\mathbf{1 2}$ \\
\hline Natural/Environmental Factors & $\mathbf{3}$ & $\mathbf{1}$ \\
\hline Submersion/Suffocation/Foreign Bodies & $\mathbf{0}$ & $\mathbf{2}$ \\
\hline Other Accidents & $\mathbf{4 1}$ & $\mathbf{7 1}$ \\
\hline Struck by an Object & $\mathbf{3}$ & $\mathbf{4}$ \\
\hline Caught Between Objects & $\mathbf{0}$ & $\mathbf{2}$ \\
\hline Cutting/Piercing Instrument/Object & $\mathbf{1}$ & $\mathbf{7}$ \\
\hline Hot, Corrosive, or Caustic Material/ & $\mathbf{0}$ & $\mathbf{1}$ \\
\hline Steam & & $\mathbf{3 9}$ \\
\hline Overexertion/Strenuous Movements & $\mathbf{1 0}$ & $\mathbf{1}$ \\
\hline Noise & $\mathbf{0}$ & $\mathbf{1 7}$ \\
\hline Repetitive Trauma & $\mathbf{2 7}$ & $\mathbf{8 8}$ \\
\hline Total & $\mathbf{5 7}$ & \\
\hline
\end{tabular}




\section{Rates of OSHA-Recordable Events}

Figure 22. OSHA-Recordable Rates by Age and Job Categories Among Women, All Diagnoses Combined

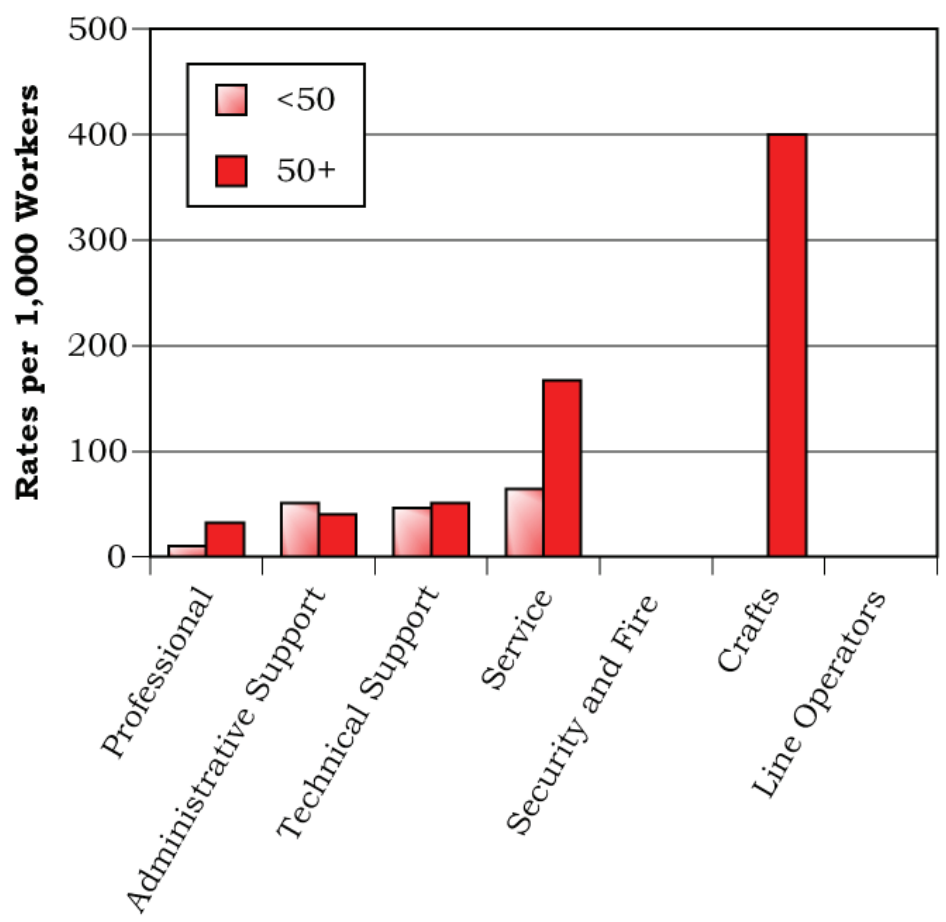

Job Category

Figure 23. OSHA-Recordable Rates by Age and Job Categories Among Men, All Diagnoses Combined

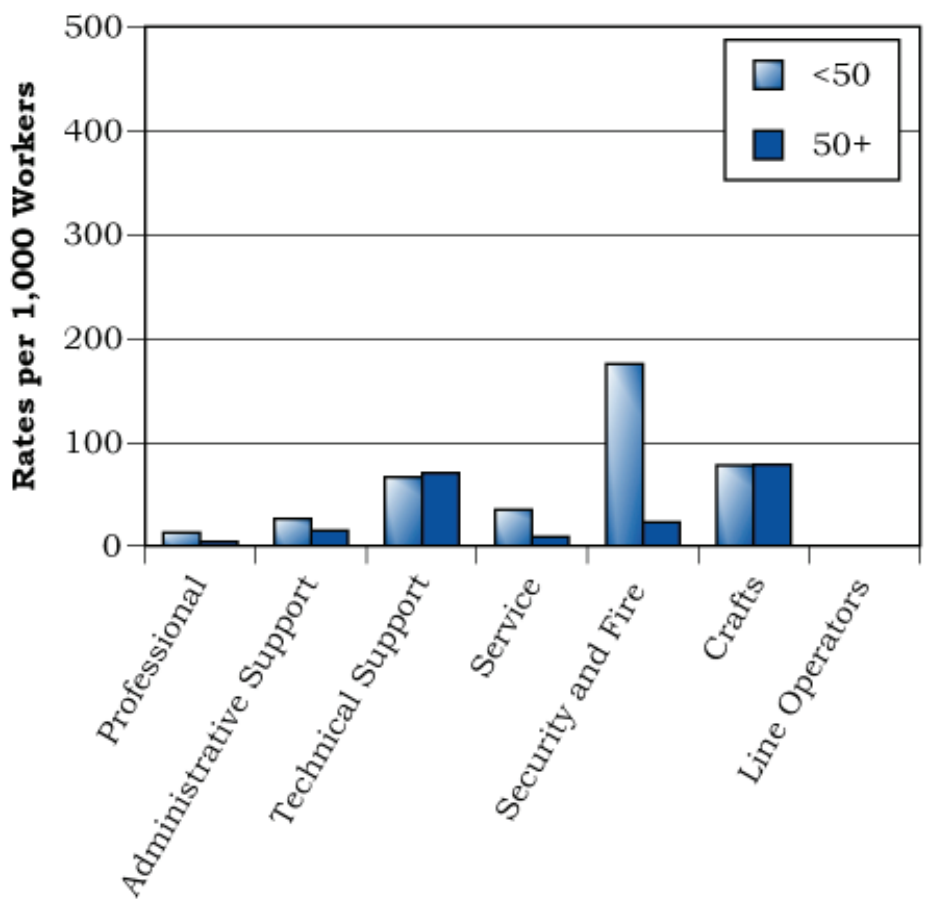

Job Category 


\section{Time Trends for OSHA-Recordable Events}

Figure 24. Age-Adjusted Rates for All OSHA-Recordable Diagnoses Combined Among Women and Men by Job Category from 2003 to 2007*

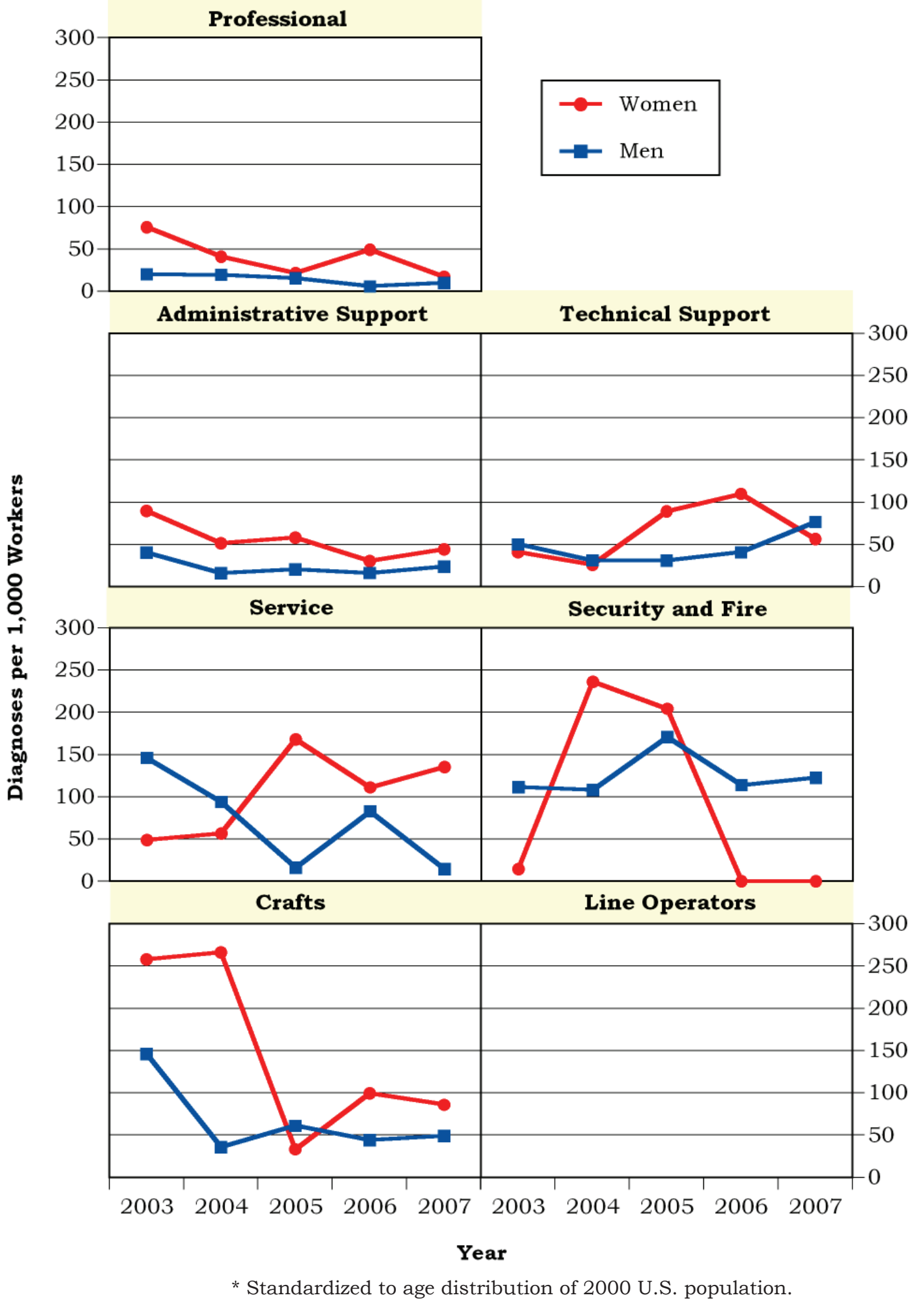




\section{Appendices}


Lawrence Livermore National Laboratory 2007

Absence Data

Appendix A. Work Force by Gender, Age, and Job Category

\begin{tabular}{|c|c|c|c|c|c|c|c|c|c|c|c|}
\hline \multirow{3}{*}{ Job Category } & \multicolumn{5}{|c|}{ Women } & \multicolumn{5}{|c|}{ Men } & \multirow[b]{3}{*}{ TOTAL } \\
\hline & \multicolumn{4}{|c|}{ Age Group } & \multirow[b]{2}{*}{ TOTAL } & \multicolumn{4}{|c|}{ Age Group } & \multirow[b]{2}{*}{ TOTAL } & \\
\hline & $16-29$ & 30 - 39 & $40-49$ & $50+$ & & $16-29$ & 30 - 39 & $40-49$ & $50+$ & & \\
\hline Professional & 44 & 108 & 251 & 185 & 588 & 132 & 426 & 896 & 1,242 & 2,696 & 3,284 \\
\hline Administrative Support & 220 & 229 & 612 & 643 & 1,704 & 324 & 168 & 408 & 812 & 1,712 & 3,416 \\
\hline Technical Support & 38 & 51 & 63 & 79 & 231 & 37 & 121 & 246 & 267 & 671 & 902 \\
\hline Service & 4 & 7 & 36 & 42 & 89 & 12 & 29 & 72 & 113 & 226 & 315 \\
\hline Security and Fire & 5 & 12 & 13 & 5 & 35 & 60 & 86 & 99 & 44 & 289 & 324 \\
\hline Crafts & 0 & 1 & 4 & 5 & 10 & 15 & 32 & 132 & 140 & 319 & 329 \\
\hline TOTAL & 311 & 408 & 979 & 959 & 2,657 & 580 & 862 & 1,853 & 2,618 & 5,913 & 8,570 \\
\hline
\end{tabular}

Appendix B. Age Distribution of the Work Force by Gender

\begin{tabular}{|c|r|r|r|r|r|r|r|r|}
\hline \multirow{3}{*}{ Year } & \multicolumn{4}{|c|}{ Women } & \multicolumn{4}{c|}{ Men } \\
\cline { 2 - 9 } & \multicolumn{3}{|c|}{$\begin{array}{c}\text { Percent Distribution by Age } \\
\text { Group }\end{array}$} & \multicolumn{3}{|c|}{$\begin{array}{c}\text { Percent Distribution by Age } \\
\text { Group }\end{array}$} \\
\cline { 2 - 9 } & $\mathbf{1 6}-\mathbf{2 9}$ & $\mathbf{3 0 - 3 9}$ & $\mathbf{4 0}-\mathbf{4 9}$ & $\mathbf{5 0}+$ & $\mathbf{1 6}-\mathbf{2 9}$ & $\mathbf{3 0}-\mathbf{3 9}$ & $\mathbf{4 0}-\mathbf{4 9}$ & $\mathbf{5 0}+$ \\
\hline $\mathbf{2 0 0 2}$ & 13.04 & 19.68 & 35.45 & 31.83 & 10.25 & 18.23 & 32.16 & 39.35 \\
\hline $\mathbf{2 0 0 3}$ & 14.40 & 16.07 & 39.17 & 30.37 & 11.31 & 15.34 & 34.82 & 38.53 \\
\hline $\mathbf{2 0 0 4}$ & 14.34 & 16.24 & 37.93 & 31.48 & 11.59 & 14.87 & 33.85 & 39.70 \\
\hline $\mathbf{2 0 0 5}$ & 12.77 & 16.87 & 33.95 & 36.41 & 10.41 & 16.04 & 28.99 & 44.56 \\
\hline $\mathbf{2 0 0 6}$ & 11.75 & 16.79 & 33.08 & 38.37 & 9.36 & 16.03 & 28.14 & 46.48 \\
\hline $\mathbf{2 0 0 7}$ & 11.70 & 15.36 & 36.85 & 36.09 & 9.81 & 14.58 & 31.34 & 44.28 \\
\hline
\end{tabular}


Lawrence Livermore National Laboratory 2007

Absence Data

Appendix C. Total Number of Workers Who Reported at Least One Absence by Gender, Age, and Job Category*

\begin{tabular}{|c|c|c|c|c|c|c|c|c|c|c|c|}
\hline \multirow{3}{*}{ Job Category } & \multicolumn{5}{|c|}{ Women } & \multicolumn{5}{|c|}{ Men } & \multirow[b]{3}{*}{ TOTAL } \\
\hline & \multicolumn{4}{|c|}{ Age Group } & \multirow[b]{2}{*}{ TOTAL } & \multicolumn{4}{|c|}{ Age Group } & \multirow[b]{2}{*}{ TOTAL } & \\
\hline & $16-29$ & 30 - 39 & $40-49$ & $50+$ & & $16-29$ & 30 - 39 & $40-49$ & $50+$ & & \\
\hline Professional & 1 & 1 & 13 & 12 & 27 & 4 & 13 & 35 & 74 & 126 & 153 \\
\hline Administrative Support & 5 & 16 & 59 & 67 & 147 & 1 & 12 & 24 & 55 & 92 & 239 \\
\hline Technical Support & 0 & 0 & 4 & 6 & 10 & 2 & 7 & 15 & 33 & 57 & 67 \\
\hline Service & 0 & 1 & 2 & 5 & 8 & 0 & 4 & 11 & 16 & 31 & 39 \\
\hline Security and Fire & 0 & 2 & 3 & 1 & 6 & 4 & 5 & 5 & 5 & 19 & 25 \\
\hline Crafts & 0 & 0 & 0 & 0 & 0 & 0 & 2 & 7 & 13 & 22 & 22 \\
\hline TOTAL & 6 & 20 & 81 & 91 & 198 & 11 & 43 & 97 & 196 & 347 & 545 \\
\hline
\end{tabular}

*Only those job categories and gender/age combinations with at least one absence appear in this table.

Appendix D. Total Number of Absences by Gender, Age, and Job Category*

\begin{tabular}{|c|c|c|c|c|c|c|c|c|c|c|c|}
\hline \multirow{3}{*}{ Job Category } & \multicolumn{5}{|c|}{ Women } & \multicolumn{5}{|c|}{ Men } & \multirow[b]{3}{*}{ TOTAL } \\
\hline & \multicolumn{4}{|c|}{ Age Group } & \multirow[b]{2}{*}{ TOTAL } & \multicolumn{4}{|c|}{ Age Group } & \multirow[b]{2}{*}{ TOTAL } & \\
\hline & $16-29$ & 30 - 39 & $40-49$ & $50+$ & & $16-29$ & 30 - 39 & $40-49$ & $50+$ & & \\
\hline Professional & 1 & 1 & 14 & 12 & 28 & 5 & 14 & 38 & 79 & 136 & 164 \\
\hline Administrative Support & 6 & 18 & 64 & 79 & 167 & 1 & 13 & 28 & 63 & 105 & 272 \\
\hline Technical Support & 0 & 0 & 4 & 6 & 10 & 2 & 9 & 15 & 36 & 62 & 72 \\
\hline Service & 0 & 1 & 3 & 5 & 9 & 0 & 5 & 11 & 16 & 32 & 41 \\
\hline Security and Fire & 0 & 2 & 4 & 1 & 7 & 4 & 5 & 6 & 5 & 20 & 27 \\
\hline Crafts & 0 & 0 & 0 & 0 & 0 & 0 & 2 & 7 & 13 & 22 & 22 \\
\hline TOTAL & 7 & 22 & 89 & 103 & 221 & 12 & 48 & 105 & 212 & 377 & 598 \\
\hline
\end{tabular}

*Only those job categories and gender/age combinations with at least one absence appear in this table. 
Lawrence Livermore National Laboratory 2007

Absence Data

Appendix E. Distribution of the Number of Calendar Days Missed per Absence by Gender and Age*

\begin{tabular}{|c|c|c|c|c|c|c|c|c|c|c|c|}
\hline \multirow{3}{*}{$\begin{array}{c}\# \\
\text { of } \\
\text { Calendar } \\
\text { Days }\end{array}$} & \multicolumn{5}{|c|}{ Women } & \multicolumn{5}{|c|}{ Men } & \multirow[b]{3}{*}{ TOTAL } \\
\hline & \multicolumn{4}{|c|}{ Age Group } & \multirow[b]{2}{*}{ TOTAL } & \multicolumn{4}{|c|}{ Age Group } & \multirow[b]{2}{*}{ TOTAL } & \\
\hline & $16-29$ & 30 - 39 & $40-49$ & $50+$ & & $16-29$ & $30-39$ & $40-49$ & $50+$ & & \\
\hline$<15$ & 4 & 13 & 45 & 52 & 114 & 8 & 27 & 70 & 119 & 224 & 338 \\
\hline $15-28$ & 3 & 1 & 12 & 24 & 40 & 1 & 9 & 19 & 42 & 71 & 111 \\
\hline $29-42$ & 0 & 1 & 10 & 7 & 18 & 2 & 6 & 5 & 18 & 31 & 49 \\
\hline $43-56$ & 0 & 2 & 7 & 6 & 15 & 0 & 3 & 2 & 7 & 12 & 27 \\
\hline $57-91$ & 0 & 2 & 9 & 7 & 18 & 1 & 2 & 5 & 15 & 23 & 41 \\
\hline $92-182$ & 0 & 2 & 4 & 3 & 9 & 0 & 1 & 2 & 10 & 13 & 22 \\
\hline $183+$ & 0 & 1 & 2 & 4 & 7 & 0 & 0 & 2 & 1 & 3 & 10 \\
\hline TOTAL & 7 & 22 & 89 & 103 & 221 & 12 & 48 & 105 & 212 & 377 & 598 \\
\hline
\end{tabular}

*Only those gender/age combinations with at least one absence appear in this table. 
Lawrence Livermore National Laboratory 2007

Absence Data

Appendix F. Distribution of the Number of Calendar Days Missed per Absence by Gender and Job Category*

Women

\begin{tabular}{|c|c|c|c|c|c|c|c|}
\hline \multirow{2}{*}{$\begin{array}{c}\# \\
\text { of } \\
\text { Calendar } \\
\text { Days }\end{array}$} & \multicolumn{6}{|c|}{ Job Category } & \multirow[b]{2}{*}{ TOTAL } \\
\hline & Professional & $\begin{array}{l}\text { Administrative } \\
\text { Support }\end{array}$ & $\begin{array}{l}\text { Technical } \\
\text { Support }\end{array}$ & Service & $\begin{array}{c}\text { Security } \\
\text { and } \\
\text { Fire }\end{array}$ & Crafts & \\
\hline$<15$ & 18 & 86 & 4 & 4 & 2 & 0 & 114 \\
\hline $15-28$ & 6 & 30 & 2 & 1 & 1 & 0 & 40 \\
\hline $29-42$ & 1 & 17 & 0 & 0 & 0 & 0 & 18 \\
\hline $43-56$ & 1 & 10 & 1 & 1 & 2 & 0 & 15 \\
\hline $57-91$ & 0 & 16 & 1 & 1 & 0 & 0 & 18 \\
\hline 92 - 182 & 0 & 4 & 1 & 2 & 2 & 0 & 9 \\
\hline $183+$ & 2 & 4 & 1 & 0 & 0 & 0 & 7 \\
\hline TOTAL & 28 & 167 & 10 & 9 & 7 & 0 & 221 \\
\hline
\end{tabular}

Men

\begin{tabular}{|c|c|c|c|c|c|c|c|}
\hline \multirow{2}{*}{$\begin{array}{c}\# \\
\text { of } \\
\text { Calendar } \\
\text { Days }\end{array}$} & \multicolumn{6}{|c|}{ Job Category } & \multirow[b]{2}{*}{ TOTAI } \\
\hline & Professional & $\begin{array}{l}\text { Administrative } \\
\text { Support }\end{array}$ & $\begin{array}{l}\text { Technical } \\
\text { Support }\end{array}$ & Service & $\begin{array}{c}\text { Security } \\
\text { and } \\
\text { Fire }\end{array}$ & Crafts & \\
\hline$<15$ & 94 & 59 & 33 & 15 & 10 & 13 & 224 \\
\hline $15-28$ & 26 & 21 & 13 & 6 & 3 & 2 & 71 \\
\hline $29-42$ & 7 & 9 & 8 & 3 & 4 & 0 & 31 \\
\hline $43-56$ & 1 & 5 & 1 & 3 & 1 & 1 & 12 \\
\hline $57-91$ & 4 & 5 & 4 & 4 & 2 & 4 & 23 \\
\hline $92-182$ & 3 & 5 & 2 & 1 & 0 & 2 & 13 \\
\hline $183+$ & 1 & 1 & 1 & 0 & 0 & 0 & 3 \\
\hline TOTAL & 136 & 105 & 62 & 32 & 20 & 22 & 377 \\
\hline
\end{tabular}

*Only those gender/job category combinations with at least one absence appear in this table. 


\section{Lawrence Livermore National Laboratory 2007}

Absence Data

Appendix G. Number of Diagnoses in Each Diagnostic Category by Gender and Age*

\begin{tabular}{|c|c|c|c|c|c|c|}
\hline & & \multicolumn{5}{|c|}{ Women } \\
\hline & & \multicolumn{4}{|c|}{ Age Group } & \multirow[b]{2}{*}{ TOTAL } \\
\hline & & $16-29$ & 30 - 39 & $40-49$ & $50+$ & \\
\hline Diagnostic Category & ICD-9-CM Code & \multirow[b]{2}{*}{2} & \multirow[b]{2}{*}{1} & \multirow[b]{2}{*}{3} & \multirow[b]{2}{*}{3} & \multirow[b]{2}{*}{9} \\
\hline INFECTIOUS \& PARASITIC DISEASES (DIS) & $001-139$ & & & & & \\
\hline -Intestinal Infectious Dis & 001-009 & 0 & 0 & 2 & 1 & 3 \\
\hline -Other Bacterial Dis & $030-041$ & 1 & 1 & 0 & 0 & 2 \\
\hline -Viral Dis with Exanthem & $050-057$ & 0 & 0 & 0 & 1 & 1 \\
\hline -Other Viral Dis \& Chlamydiae & 070-079 & 1 & 0 & 1 & 1 & 3 \\
\hline -Other Infections \& Parasitic Dis & 130-136 & 0 & 0 & 0 & 0 & 0 \\
\hline MALIGNANT NEOPLASMS & $140-208,230-234$ & 0 & 0 & 5 & 11 & 16 \\
\hline -Digestive \& Peritoneal & $150-159$ & 0 & 0 & 0 & 0 & 0 \\
\hline -Bone, Connective Tissue, Skin & 170-173, 176 & 0 & 0 & 0 & 0 & 0 \\
\hline -Breast & 174-175 & 0 & 0 & 4 & 8 & 12 \\
\hline -Genitourinary & 179-189 & 0 & 0 & 1 & 3 & 4 \\
\hline -Lymphatic \& Hematopoietic & $200-208$ & 0 & 0 & 0 & 0 & 0 \\
\hline BENIGN \& UNCERTAIN NEOPLASMS & 210-229, 235-239 & 0 & 4 & 5 & 4 & 13 \\
\hline ENDOCRINE/METABOLIC/IMMUNITY & $240-279$ & 0 & 0 & 2 & 1 & 3 \\
\hline -Thyroid Gland Disorders & $240-246$ & 0 & 0 & 0 & 0 & 0 \\
\hline -Other Endocrine Gland Dis & $250-259$ & 0 & 0 & 0 & 0 & 0 \\
\hline -Other Metabolic \& Immunity Disorders & 270-279 & 0 & 0 & 2 & 1 & 3 \\
\hline BLOOD \& BLOOD-FORMING ORGANS & $280-289$ & 0 & 0 & 1 & 1 & 2 \\
\hline MENTAL DISORDERS & 290-319 & 0 & 3 & 1 & 1 & 5 \\
\hline -Non-Psychotic Disorders & $300-302,306-316$ & 0 & 3 & 1 & 1 & 5 \\
\hline NERVOUS SYSTEM (NS) \& SENSE ORGANS & 320-389 & 2 & 0 & 9 & 7 & 18 \\
\hline -Hereditary/Degenerative Central NS Dis & 330-337 & 0 & 0 & 0 & 0 & 0 \\
\hline -Other Disorders of Central NS & $340-349$ & 0 & 0 & 0 & 0 & 0 \\
\hline -Disorders of Peripheral NS & 350-359 & 0 & 0 & 2 & 2 & 4 \\
\hline -Disorders of Eye & $360-379$ & 0 & 0 & 1 & 5 & 6 \\
\hline -Dis of Ear \& Mastoid & $380-389$ & 2 & 0 & 6 & 0 & 8 \\
\hline CIRCULATORY SYSTEM & $390-459$ & 0 & 0 & 3 & 4 & 7 \\
\hline -Chronic Rheumatic Heart Dis & 393-398 & 0 & 0 & 0 & 0 & 0 \\
\hline -Hypertensive Dis & $401-405$ & 0 & 0 & 0 & 1 & 1 \\
\hline -Ischemic Heart Dis & $410-414$ & 0 & 0 & 0 & 1 & 1 \\
\hline -Other Heart Dis & $420-429$ & 0 & 0 & 0 & 0 & 0 \\
\hline -Cerebrovascular Dis & $430-438$ & 0 & 0 & 0 & 1 & 1 \\
\hline -Dis of Arteries \& Capillaries & $440-448$ & 0 & 0 & 2 & 1 & 3 \\
\hline -Dis of Veins, Lymphatics, Other & $451-459$ & 0 & 0 & 1 & 0 & 1 \\
\hline RESPIRATORY SYSTEM & $460-519$ & 1 & 4 & 18 & 14 & 37 \\
\hline -Acute Respiratory Infections & $460-466$ & 0 & 3 & 11 & 5 & 19 \\
\hline -Other Dis Upper Respiratory Tract & $470-478$ & 0 & 1 & 1 & 2 & 4 \\
\hline
\end{tabular}

(Continued)

*Only those diagnostic categories and gender/age combinations with at least one occurrence appear in this table. 


\section{Lawrence Livermore National Laboratory 2007}

Absence Data

Appendix G. Number of Diagnoses in Each Diagnostic Category by Gender and Age*

\begin{tabular}{|c|c|c|c|c|c|c|c|}
\hline & & \multicolumn{5}{|c|}{ Men } & \multirow[b]{3}{*}{ TOTAL } \\
\hline & & \multicolumn{4}{|c|}{ Age Group } & \multirow[b]{2}{*}{ TOTAL } & \\
\hline & & $16-29$ & 30 - 39 & $40-49$ & $50+$ & & \\
\hline Diagnostic Category & ICD-9-CM Code & & & & & & \\
\hline INFECTIOUS \& PARASITIC DISEASES (DIS) & 001-139 & 2 & 7 & 11 & 15 & 35 & 44 \\
\hline -Intestinal Infectious Dis & 001-009 & 0 & 2 & 1 & 4 & 7 & 10 \\
\hline -Other Bacterial Dis & 030-041 & 0 & 2 & 2 & 4 & 8 & 10 \\
\hline -Viral Dis with Exanthem & 050-057 & 0 & 1 & 2 & 0 & 3 & 4 \\
\hline -Other Viral Dis \& Chlamydiae & 070-079 & 2 & 2 & 6 & 5 & 15 & 18 \\
\hline -Other Infections \& Parasitic Dis & $130-136$ & 0 & 0 & 0 & 2 & 2 & 2 \\
\hline MALIGNANT NEOPLASMS & $140-208,230-234$ & 0 & 0 & 2 & 14 & 16 & 32 \\
\hline -Digestive \& Peritoneal & 150-159 & 0 & 0 & 0 & 2 & 2 & 2 \\
\hline -Bone, Connective Tissue, Skin & 170-173, 176 & 0 & 0 & 0 & 3 & 3 & 3 \\
\hline -Breast & 174-175 & 0 & 0 & 0 & 0 & 0 & 12 \\
\hline -Genitourinary & 179-189 & 0 & 0 & 1 & 9 & 10 & 14 \\
\hline -Lymphatic \& Hematopoietic & $200-208$ & 0 & 0 & 1 & 0 & 1 & 1 \\
\hline BENIGN \& UNCERTAIN NEOPLASMS & 210-229, 235-239 & 0 & 1 & 2 & 4 & 7 & 20 \\
\hline ENDOCRINE/METABOLIC/IMMUNITY & $240-279$ & 0 & 0 & 3 & 3 & 6 & 9 \\
\hline -Thyroid Gland Disorders & $240-246$ & 0 & 0 & 1 & 0 & 1 & 1 \\
\hline -Other Endocrine Gland Dis & $250-259$ & 0 & 0 & 0 & 2 & 2 & 2 \\
\hline -Other Metabolic \& Immunity Disorders & $270-279$ & 0 & 0 & 2 & 1 & 3 & 6 \\
\hline BLOOD \& BLOOD-FORMING ORGANS & $280-289$ & 0 & 1 & 0 & 0 & 1 & 3 \\
\hline MENTAL DISORDERS & 290-319 & 0 & 1 & 1 & 1 & 3 & 8 \\
\hline -Non-Psychotic Disorders & $300-302,306-316$ & 0 & 1 & 1 & 1 & 3 & 8 \\
\hline NERVOUS SYSTEM (NS) \& SENSE ORGANS & 320-389 & 2 & 2 & 7 & 12 & 23 & 41 \\
\hline -Hereditary/Degenerative Central NS Dis & $330-337$ & 0 & 0 & 1 & 0 & 1 & 1 \\
\hline -Other Disorders of Central NS & $340-349$ & 0 & 0 & 0 & 1 & 1 & 1 \\
\hline -Disorders of Peripheral NS & 350-359 & 0 & 1 & 2 & 2 & 5 & 9 \\
\hline -Disorders of Eye & $360-379$ & 2 & 1 & 2 & 8 & 13 & 19 \\
\hline -Dis of Ear \& Mastoid & 380-389 & 0 & 0 & 2 & 1 & 3 & 11 \\
\hline CIRCULATORY SYSTEM & $390-459$ & 0 & 3 & 5 & 18 & 26 & 33 \\
\hline -Chronic Rheumatic Heart Dis & 393-398 & 0 & 0 & 1 & 0 & 1 & 1 \\
\hline -Hypertensive Dis & $401-405$ & 0 & 0 & 0 & 1 & 1 & 2 \\
\hline -Ischemic Heart Dis & $410-414$ & 0 & 2 & 0 & 5 & 7 & 8 \\
\hline -Other Heart Dis & $420-429$ & 0 & 1 & 1 & 5 & 7 & 7 \\
\hline -Cerebrovascular Dis & $430-438$ & 0 & 0 & 0 & 1 & 1 & 2 \\
\hline -Dis of Arteries \& Capillaries & $440-448$ & 0 & 0 & 0 & 2 & 2 & 5 \\
\hline -Dis of Veins, Lymphatics, Other & $451-459$ & 0 & 0 & 3 & 4 & 7 & 8 \\
\hline RESPIRATORY SYSTEM & $460-519$ & 6 & 13 & 19 & 44 & 82 & 119 \\
\hline -Acute Respiratory Infections & $460-466$ & 4 & 1 & 5 & 21 & 31 & 50 \\
\hline -Other Dis Upper Respiratory Tract & $470-478$ & 0 & 2 & 4 & 6 & 12 & 16 \\
\hline
\end{tabular}

(Continued)

*Only those diagnostic categories and gender/age combinations with at least one occurrence appear in this table. 


\section{Lawrence Livermore National Laboratory 2007}

Absence Data

Appendix G. Number of Diagnoses in Each Diagnostic Category by Gender and Age*

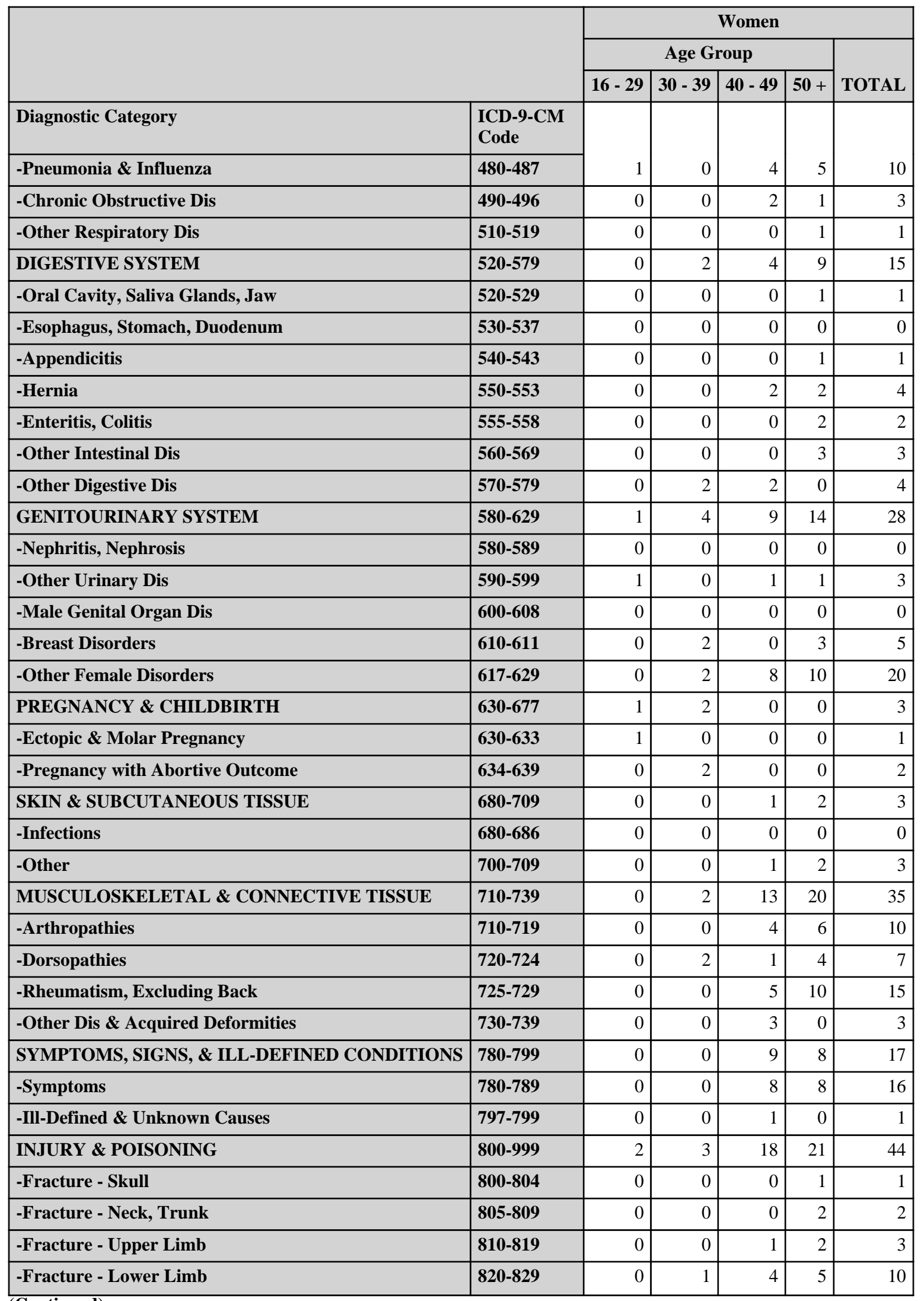

(Continued)

*Only those diagnostic categories and gender/age combinations with at least one occurrence appear in this table. 


\section{Lawrence Livermore National Laboratory 2007}

Absence Data

Appendix G. Number of Diagnoses in Each Diagnostic Category by Gender and Age*

\begin{tabular}{|c|c|c|c|c|c|c|c|}
\hline & & \multicolumn{5}{|c|}{ Men } & \multirow[b]{3}{*}{ TOTAL } \\
\hline & & \multicolumn{4}{|c|}{ Age Group } & \multirow[b]{2}{*}{ TOTAL } & \\
\hline & & $16-29$ & 30 - 39 & $40-49$ & $50+$ & & \\
\hline Diagnostic Category & $\begin{array}{l}\text { ICD-9-CM } \\
\text { Code }\end{array}$ & & & & & & \\
\hline -Pneumonia \& Influenza & $480-487$ & 1 & 4 & 6 & 15 & 26 & 36 \\
\hline -Chronic Obstructive Dis & $490-496$ & 1 & 4 & 2 & 1 & 8 & 11 \\
\hline -Other Respiratory Dis & $510-519$ & 0 & 2 & 2 & 1 & 5 & 6 \\
\hline DIGESTIVE SYSTEM & $520-579$ & 0 & 9 & 20 & 26 & 55 & 70 \\
\hline -Oral Cavity, Saliva Glands, Jaw & $520-529$ & 0 & 3 & 2 & 0 & 5 & 6 \\
\hline -Esophagus, Stomach, Duodenum & $530-537$ & 0 & 0 & 3 & 1 & 4 & 4 \\
\hline -Appendicitis & $540-543$ & 0 & 0 & 0 & 2 & 2 & 3 \\
\hline -Hernia & $550-553$ & 0 & 1 & 6 & 15 & 22 & 26 \\
\hline -Enteritis, Colitis & $555-558$ & 0 & 1 & 2 & 3 & 6 & 8 \\
\hline -Other Intestinal Dis & $560-569$ & 0 & 2 & 2 & 3 & 7 & 10 \\
\hline -Other Digestive Dis & $570-579$ & 0 & 2 & 5 & 2 & 9 & 13 \\
\hline GENITOURINARY SYSTEM & $580-629$ & 0 & 0 & 4 & 6 & 10 & 38 \\
\hline -Nephritis, Nephrosis & $580-589$ & 0 & 0 & 1 & 1 & 2 & 2 \\
\hline -Other Urinary Dis & $590-599$ & 0 & 0 & 1 & 2 & 3 & 6 \\
\hline -Male Genital Organ Dis & $600-608$ & 0 & 0 & 2 & 2 & 4 & 4 \\
\hline -Breast Disorders & $610-611$ & 0 & 0 & 0 & 1 & 1 & 6 \\
\hline -Other Female Disorders & $617-629$ & 0 & 0 & 0 & 0 & 0 & 20 \\
\hline PREGNANCY \& CHILDBIRTH & $630-677$ & 0 & 0 & 0 & 0 & 0 & 3 \\
\hline -Ectopic \& Molar Pregnancy & $630-633$ & 0 & 0 & 0 & 0 & 0 & 1 \\
\hline -Pregnancy with Abortive Outcome & $634-639$ & 0 & 0 & 0 & 0 & 0 & 2 \\
\hline SKIN \& SUBCUTANEOUS TISSUE & $680-709$ & 0 & 1 & 0 & 5 & 6 & 9 \\
\hline -Infections & $680-686$ & 0 & 1 & 0 & 5 & 6 & 6 \\
\hline -Other & 700-709 & 0 & 0 & 0 & 0 & 0 & 3 \\
\hline MUSCULOSKELETAL \& CONNECTIVE TISSUE & $710-739$ & 3 & 3 & 18 & 40 & 64 & 99 \\
\hline -Arthropathies & $710-719$ & 0 & 0 & 3 & 16 & 19 & 29 \\
\hline -Dorsopathies & $720-724$ & 3 & 3 & 10 & 14 & 30 & 37 \\
\hline -Rheumatism, Excluding Back & 725-729 & 0 & 0 & 3 & 9 & 12 & 27 \\
\hline -Other Dis \& Acquired Deformities & $730-739$ & 0 & 0 & 2 & 1 & 3 & $\overline{6}$ \\
\hline SYMPTOMS, SIGNS, \& ILL-DEFINED CONDITIONS & $780-799$ & 1 & 1 & 7 & 19 & 28 & 45 \\
\hline -Symptoms & $780-789$ & 1 & 1 & 7 & 19 & 28 & 44 \\
\hline -Ill-Defined \& Unknown Causes & 797-799 & 0 & 0 & 0 & 0 & 0 & 1 \\
\hline INJURY \& POISONING & $800-999$ & 5 & 13 & 19 & 38 & 75 & 119 \\
\hline -Fracture - Skull & $800-804$ & 0 & 0 & 0 & 0 & 0 & 1 \\
\hline -Fracture - Neck, Trunk & $805-809$ & 0 & 0 & 0 & 2 & 2 & 4 \\
\hline -Fracture - Upper Limb & 810-819 & 0 & 4 & 1 & 1 & 6 & 9 \\
\hline -Fracture - Lower Limb & $820-829$ & 2 & 0 & 2 & 3 & 7 & 17 \\
\hline
\end{tabular}

(Continued)

*Only those diagnostic categories and gender/age combinations with at least one occurrence appear in this table. 


\section{Lawrence Livermore National Laboratory 2007}

Absence Data

Appendix G. Number of Diagnoses in Each Diagnostic Category by Gender and Age*

\begin{tabular}{|c|c|c|c|c|c|c|}
\hline & & \multicolumn{5}{|c|}{ Women } \\
\hline & & \multicolumn{4}{|c|}{ Age Group } & \multirow[b]{2}{*}{ TOTAL } \\
\hline & & $16-29$ & 30 - 39 & 40 - 49 & $50+$ & \\
\hline Diagnostic Category & $\begin{array}{l}\text { ICD-9-CM } \\
\text { Code }\end{array}$ & & & & & \\
\hline -Dislocation & 830-839 & 0 & 0 & 3 & 4 & 7 \\
\hline -Sprains \& Strains - Back & 846-847 & 0 & 1 & 2 & 0 & 3 \\
\hline -Sprains \& Strains - Other & $840-845,848$ & 0 & 1 & 3 & 5 & 9 \\
\hline -Internal Injury - Thorax, Abdomen, Pelvis & $860-869$ & 0 & 0 & 1 & 0 & 1 \\
\hline -Open Wound - Head, Neck, Trunk & 870-879 & 0 & 0 & 0 & 1 & 1 \\
\hline -Open Wound - Upper Limb & 880-887 & 0 & 0 & 0 & 0 & 0 \\
\hline -Open Wound - Lower Limb & $890-897$ & 1 & 0 & 0 & 0 & 1 \\
\hline -Superficial Injury & $910-919$ & 0 & 0 & 0 & 0 & 0 \\
\hline -Contusion & $920-924$ & 0 & 0 & 0 & 0 & 0 \\
\hline -Burns & 940-949 & 0 & 0 & 0 & 0 & 0 \\
\hline -Complications \& Unspecified Injuries & 958-959 & 0 & 0 & 1 & 1 & 2 \\
\hline -Unspecified Effects - External Causes & $990-995$ & 0 & 0 & 0 & 0 & 0 \\
\hline -Complications of Surgical/Medical Care & 996-999 & 1 & 0 & 3 & 0 & 4 \\
\hline HEALTH STATUS/HEALTH SERVICE CONTACT & V01-V82 & 0 & 1 & 1 & 4 & 6 \\
\hline -Isolation \& Prophylactic Measures & V07-V09 & 0 & 0 & 0 & 0 & 0 \\
\hline -Personal \& Family History & V10-V19 & 0 & 0 & 0 & 0 & 0 \\
\hline -Health Services Reproduction/Development & V20-V29 & 0 & 0 & 0 & 0 & 0 \\
\hline -Health Status & V40-V49 & 0 & 0 & 0 & 0 & 0 \\
\hline -Specific Procedures/Aftercare & V50-V59 & 0 & 1 & 1 & 4 & 6 \\
\hline -Other Circumstances & V60-V69 & 0 & 0 & 0 & 0 & 0 \\
\hline
\end{tabular}

*Only those diagnostic categories and gender/age combinations with at least one occurrence appear in this table. 


\section{Lawrence Livermore National Laboratory 2007}

Absence Data

Appendix G. Number of Diagnoses in Each Diagnostic Category by Gender and Age*

\begin{tabular}{|c|c|c|c|c|c|c|c|}
\hline & & \multicolumn{5}{|c|}{ Men } & \multirow[b]{3}{*}{ TOTAL } \\
\hline & & \multicolumn{4}{|c|}{ Age Group } & \multirow[b]{2}{*}{ TOTAL } & \\
\hline & & $16-29$ & $30-39$ & $40-49$ & $50+$ & & \\
\hline Diagnostic Category & $\begin{array}{l}\text { ICD-9-CM } \\
\text { Code }\end{array}$ & & & & & & \\
\hline -Dislocation & 830-839 & 0 & 3 & 2 & 7 & 12 & 19 \\
\hline -Sprains \& Strains - Back & 846-847 & 1 & 1 & 0 & 7 & 9 & 12 \\
\hline -Sprains \& Strains - Other & $840-845,848$ & 0 & 3 & 12 & 9 & 24 & 33 \\
\hline -Internal Injury - Thorax, Abdomen, Pelvis & $860-869$ & 0 & 0 & 0 & 0 & 0 & 1 \\
\hline -Open Wound - Head, Neck, Trunk & $870-879$ & 0 & 0 & 0 & 0 & 0 & 1 \\
\hline -Open Wound - Upper Limb & $880-887$ & 1 & 0 & 0 & 0 & 1 & 1 \\
\hline -Open Wound - Lower Limb & $890-897$ & 1 & 0 & 0 & 2 & 3 & 4 \\
\hline -Superficial Injury & $910-919$ & 0 & 0 & 0 & 1 & 1 & 1 \\
\hline -Contusion & $920-924$ & 0 & 0 & 1 & 1 & 2 & 2 \\
\hline -Burns & $940-949$ & 0 & 0 & 0 & 2 & 2 & 2 \\
\hline -Complications \& Unspecified Injuries & 958-959 & 0 & 2 & 0 & 1 & 3 & 5 \\
\hline -Unspecified Effects - External Causes & $990-995$ & 0 & 0 & 1 & 1 & 2 & 2 \\
\hline -Complications of Surgical/Medical Care & 996-999 & 0 & 0 & 0 & 1 & 1 & 5 \\
\hline HEALTH STATUS/HEALTH SERVICE CONTACT & V01-V82 & 1 & 1 & 3 & 3 & 8 & 14 \\
\hline -Isolation \& Prophylactic Measures & V07-V09 & 0 & 1 & 0 & 0 & 1 & 1 \\
\hline -Personal \& Family History & V10-V19 & 0 & 0 & 0 & 1 & 1 & 1 \\
\hline -Health Services Reproduction/Development & V20-V29 & 1 & 0 & 1 & 0 & 2 & 2 \\
\hline -Health Status & V40-V49 & 0 & 0 & 0 & 1 & 1 & 1 \\
\hline -Specific Procedures/Aftercare & V50-V59 & 0 & 0 & 1 & 1 & 2 & 8 \\
\hline -Other Circumstances & V60-V69 & 0 & 0 & 1 & 0 & 1 & 1 \\
\hline
\end{tabular}

\begin{tabular}{|c|c|c|c|c|c|c|c|c|c|c|c|}
\hline & \multicolumn{5}{|c|}{ Women } & \multicolumn{5}{|c|}{ Men } & \multirow[b]{3}{*}{ TOTAL } \\
\hline & \multicolumn{4}{|c|}{ Age Group } & \multirow[b]{2}{*}{ TOTAL } & \multicolumn{4}{|c|}{ Age Group } & \multirow[b]{2}{*}{ TOTAL } & \\
\hline & $16-29$ & 30 - 39 & $40-49$ & $50+$ & & $16-29$ & $30-39$ & $40-49$ & $50+$ & & \\
\hline $\begin{array}{l}\text { Diagnostic } \\
\text { Category }\end{array}$ & & & & & & & & & & & \\
\hline Total & 9 & 26 & 102 & 124 & 261 & 20 & 56 & 121 & 248 & 445 & 706 \\
\hline
\end{tabular}

*Only those diagnostic categories and gender/age combinations with at least one occurrence appear in this table. 
Lawrence Livermore National Laboratory 2007

Absence Data

Appendix H. Total Number of Calendar Days Absent in Each Diagnostic Category by Gender and Age*

\begin{tabular}{|c|c|c|c|c|c|c|}
\hline & & \multicolumn{5}{|c|}{ Women } \\
\hline & & \multicolumn{4}{|c|}{ Age Group } & \multirow[b]{2}{*}{ TOTAL } \\
\hline & & $16-29$ & 30 - 39 & $40-49$ & $50+$ & \\
\hline Diagnostic Category & ICD-9-CM Code & \multirow[b]{2}{*}{6} & \multirow[b]{2}{*}{6} & \multirow[b]{2}{*}{20} & \multirow[b]{2}{*}{17} & \multirow[b]{2}{*}{49} \\
\hline INFECTIOUS \& PARASITIC DISEASES (DIS) & 001-139 & & & & & \\
\hline MALIGNANT NEOPLASMS & $140-208,230-234$ & 0 & 0 & 359 & 460 & 819 \\
\hline BENIGN \& UNCERTAIN NEOPLASMS & 210-229, 235-239 & 0 & 178 & 206 & 216 & 600 \\
\hline ENDOCRINE/METABOLIC/IMMUNITY & $240-279$ & 0 & 0 & 123 & 9 & 132 \\
\hline BLOOD \& BLOOD-FORMING ORGANS & 280-289 & 0 & 0 & 14 & 426 & 440 \\
\hline MENTAL DISORDERS & 290-319 & 0 & 425 & 14 & 15 & 454 \\
\hline NERVOUS SYSTEM (NS) \& SENSE ORGANS & $320-389$ & 18 & 0 & 131 & 50 & 199 \\
\hline CIRCULATORY SYSTEM & $390-459$ & 0 & 0 & 102 & 97 & 199 \\
\hline RESPIRATORY SYSTEM & $460-519$ & 9 & 18 & 227 & 228 & 482 \\
\hline DIGESTIVE SYSTEM & $520-579$ & 0 & 62 & 108 & 609 & 779 \\
\hline GENITOURINARY SYSTEM & $580-629$ & 8 & 321 & 313 & 289 & 931 \\
\hline PREGNANCY \& CHILDBIRTH & $630-677$ & 27 & 40 & 0 & 0 & 67 \\
\hline SKIN \& SUBCUTANEOUS TISSUE & $680-709$ & 0 & 0 & 20 & 57 & 77 \\
\hline MUSCULOSKELETAL \& CONNECTIVE TISSUE & 710-739 & 0 & 19 & 953 & 1,162 & 2,134 \\
\hline SYMPTOMS, SIGNS, \& ILL-DEFINED CONDITIONS & $780-799$ & 0 & 0 & 332 & 49 & 381 \\
\hline INJURY \& POISONING & 800-999 & 27 & 447 & 419 & 914 & 1,807 \\
\hline
\end{tabular}

\footnotetext{
*Absences with >1 ICD-9-CM code in the same diagnostic category were counted only once. Only those diagnostic categories and gender/age combinations with at least one occurrence appear in this table.
} 
Lawrence Livermore National Laboratory 2007

Absence Data

Appendix H. Total Number of Calendar Days Absent in Each Diagnostic Category by Gender and Age*

\begin{tabular}{|c|c|c|c|c|c|c|c|}
\hline & & \multicolumn{5}{|c|}{ Men } & \multirow[b]{3}{*}{ TOTAL } \\
\hline & & \multicolumn{4}{|c|}{ Age Group } & \multirow[b]{2}{*}{ TOTAL } & \\
\hline & & $16-29$ & 30 - 39 & $40-49$ & $\mathbf{5 0 +}$ & & \\
\hline Diagnostic Category & ICD-9-CM Code & & & & & & \\
\hline INFECTIOUS \& PARASITIC DISEASES (DIS) & $001-139$ & 17 & 83 & 92 & 519 & 711 & 760 \\
\hline MALIGNANT NEOPLASMS & $140-208,230-234$ & 0 & 0 & 214 & 741 & 955 & 1,774 \\
\hline BENIGN \& UNCERTAIN NEOPLASMS & 210-229, 235-239 & 0 & 32 & 28 & 135 & 195 & 795 \\
\hline ENDOCRINE/METABOLIC/IMMUNITY & $240-279$ & 0 & 0 & 36 & 160 & 196 & 328 \\
\hline BLOOD \& BLOOD-FORMING ORGANS & $280-289$ & 0 & 66 & 0 & 0 & 66 & 506 \\
\hline MENTAL DISORDERS & \begin{tabular}{|l|}
$290-319$ \\
\end{tabular} & 0 & 31 & 9 & 43 & 83 & 537 \\
\hline NERVOUS SYSTEM (NS) \& SENSE ORGANS & $320-389$ & 109 & 22 & 106 & 429 & 666 & 865 \\
\hline CIRCULATORY SYSTEM & $390-459$ & 0 & 78 & 204 & 648 & 930 & 1,129 \\
\hline RESPIRATORY SYSTEM & $460-519$ & 32 & 114 & 140 & 429 & 715 & 1,197 \\
\hline DIGESTIVE SYSTEM & $520-579$ & 0 & 155 & 405 & 536 & 1,096 & 1,875 \\
\hline GENITOURINARY SYSTEM & $580-629$ & 0 & 0 & 221 & 75 & 296 & 1,227 \\
\hline PREGNANCY \& CHILDBIRTH & $630-677$ & 0 & 0 & 0 & 0 & 0 & 67 \\
\hline SKIN \& SUBCUTANEOUS TISSUE & $680-709$ & 0 & 4 & 0 & 207 & 211 & 288 \\
\hline MUSCULOSKELETAL \& CONNECTIVE TISSUE & 710-739 & 11 & 169 & 573 & 1,155 & 1,908 & 4,042 \\
\hline SYMPTOMS, SIGNS, \& ILL-DEFINED CONDITIONS & $780-799$ & 3 & 6 & 52 & 324 & 385 & 766 \\
\hline INJURY \& POISONING & \begin{tabular}{|c|}
$800-999$ \\
\end{tabular} & 71 & 255 & 410 & 1,219 & 1,955 & 3,762 \\
\hline
\end{tabular}

\footnotetext{
*Absences with >1 ICD-9-CM code in the same diagnostic category were counted only once. Only those diagnostic categories and gender/age combinations with at least one occurrence appear in this table.
} 


\section{Lawrence Livermore National Laboratory 2007}

\section{Absence Data}

Appendix I. Number of Diagnoses in Each Diagnostic Category by Gender and Job Category*

\begin{tabular}{|c|c|c|c|c|c|c|c|}
\hline & & \multicolumn{6}{|c|}{ Women } \\
\hline & & \multicolumn{5}{|c|}{ Job Category } & \multirow[b]{2}{*}{ TOTAL } \\
\hline & & Professional & $\begin{array}{c}\text { Administrative } \\
\text { Support }\end{array}$ & $\begin{array}{l}\text { Technical } \\
\text { Support }\end{array}$ & Service & $\begin{array}{c}\text { Security } \\
\text { and } \\
\text { Fire }\end{array}$ & \\
\hline Diagnostic Category & $\begin{array}{l}\text { ICD-9-CM } \\
\text { Code }\end{array}$ & & & & & & \\
\hline INFECTIOUS \& PARASITIC DISEASES (DIS) & 001-139 & 3 & 6 & 0 & 0 & 0 & 9 \\
\hline -Intestinal Infectious Dis & 001-009 & 2 & 1 & 0 & 0 & 0 & 3 \\
\hline -Other Bacterial Dis & 030-041 & 0 & 2 & 0 & 0 & 0 & 2 \\
\hline -Viral Dis with Exanthem & 050-057 & 0 & 1 & 0 & 0 & 0 & 1 \\
\hline -Other Viral Dis \& Chlamydiae & 070-079 & 1 & 2 & 0 & 0 & 0 & 3 \\
\hline MALIGNANT NEOPLASMS & $\begin{array}{l}140-208 \\
230-234\end{array}$ & 0 & 13 & 1 & 0 & 2 & 16 \\
\hline -Breast & 174-175 & 0 & 11 & 1 & 0 & 0 & 12 \\
\hline -Genitourinary & 179-189 & 0 & 2 & 0 & 0 & 2 & 4 \\
\hline BENIGN \& UNCERTAIN NEOPLASMS & $\begin{array}{l}\text { 210-229, } \\
\text { 235-239 }\end{array}$ & 1 & 10 & 1 & 1 & 0 & 13 \\
\hline ENDOCRINE/METABOLIC/IMMUNITY & $240-279$ & 0 & 3 & 0 & 0 & 0 & 3 \\
\hline -Other Metabolic \& Immunity Disorders & 270-279 & 0 & 3 & 0 & 0 & 0 & 3 \\
\hline BLOOD \& BLOOD-FORMING ORGANS & $280-289$ & 1 & 1 & 0 & 0 & 0 & 2 \\
\hline MENTAL DISORDERS & $290-319$ & 0 & 5 & 0 & 0 & 0 & 5 \\
\hline -Non-Psychotic Disorders & $\begin{array}{l}300-302 \\
306-316\end{array}$ & 0 & 5 & 0 & 0 & 0 & 5 \\
\hline NERVOUS SYSTEM (NS) \& SENSE ORGANS & 320-389 & 3 & 15 & 0 & 0 & 0 & 18 \\
\hline -Disorders of Peripheral NS & $350-359$ & 1 & 3 & 0 & 0 & 0 & 4 \\
\hline -Disorders of Eye & $360-379$ & 1 & 5 & 0 & 0 & 0 & 6 \\
\hline -Dis of Ear \& Mastoid & 380-389 & 1 & 7 & 0 & 0 & 0 & 8 \\
\hline CIRCULATORY SYSTEM & $390-459$ & 1 & 5 & 1 & 0 & 0 & 7 \\
\hline -Hypertensive Dis & $401-405$ & 0 & 1 & 0 & 0 & 0 & 1 \\
\hline -Ischemic Heart Dis & $410-414$ & 0 & 1 & 0 & 0 & 0 & 1 \\
\hline -Cerebrovascular Dis & $430-438$ & 1 & 0 & 0 & 0 & 0 & 1 \\
\hline -Dis of Arteries \& Capillaries & $440-448$ & 0 & 3 & 0 & 0 & 0 & 3 \\
\hline -Dis of Veins, Lymphatics, Other & 451-459 & 0 & 0 & 1 & 0 & 0 & 1 \\
\hline RESPIRATORY SYSTEM & $460-519$ & 7 & 23 & 2 & 3 & 2 & 37 \\
\hline -Acute Respiratory Infections & $460-466$ & 4 & 11 & 0 & 2 & 2 & 19 \\
\hline -Other Dis Upper Respiratory Tract & $470-478$ & 1 & 2 & 0 & 1 & 0 & 4 \\
\hline -Pneumonia \& Influenza & $480-487$ & 2 & 7 & 1 & 0 & 0 & 10 \\
\hline -Chronic Obstructive Dis & $490-496$ & 0 & 2 & 1 & 0 & 0 & 3 \\
\hline -Other Respiratory Dis & $510-519$ & 0 & 1 & 0 & 0 & 0 & 1 \\
\hline DIGESTIVE SYSTEM & $520-579$ & 3 & 10 & 1 & 1 & 0 & 15 \\
\hline -Oral Cavity, Saliva Glands, Jaw & $520-529$ & 0 & 0 & 1 & 0 & 0 & 1 \\
\hline -Appendicitis & $540-543$ & 0 & 1 & 0 & 0 & 0 & 1 \\
\hline
\end{tabular}

(Continued)

*Only those diagnostic categories and gender/job category combinations with at least one occurrence appear in this table. 


\section{Lawrence Livermore National Laboratory 2007}

Absence Data

Appendix I. Number of Diagnoses in Each Diagnostic Category by Gender and Job Category*

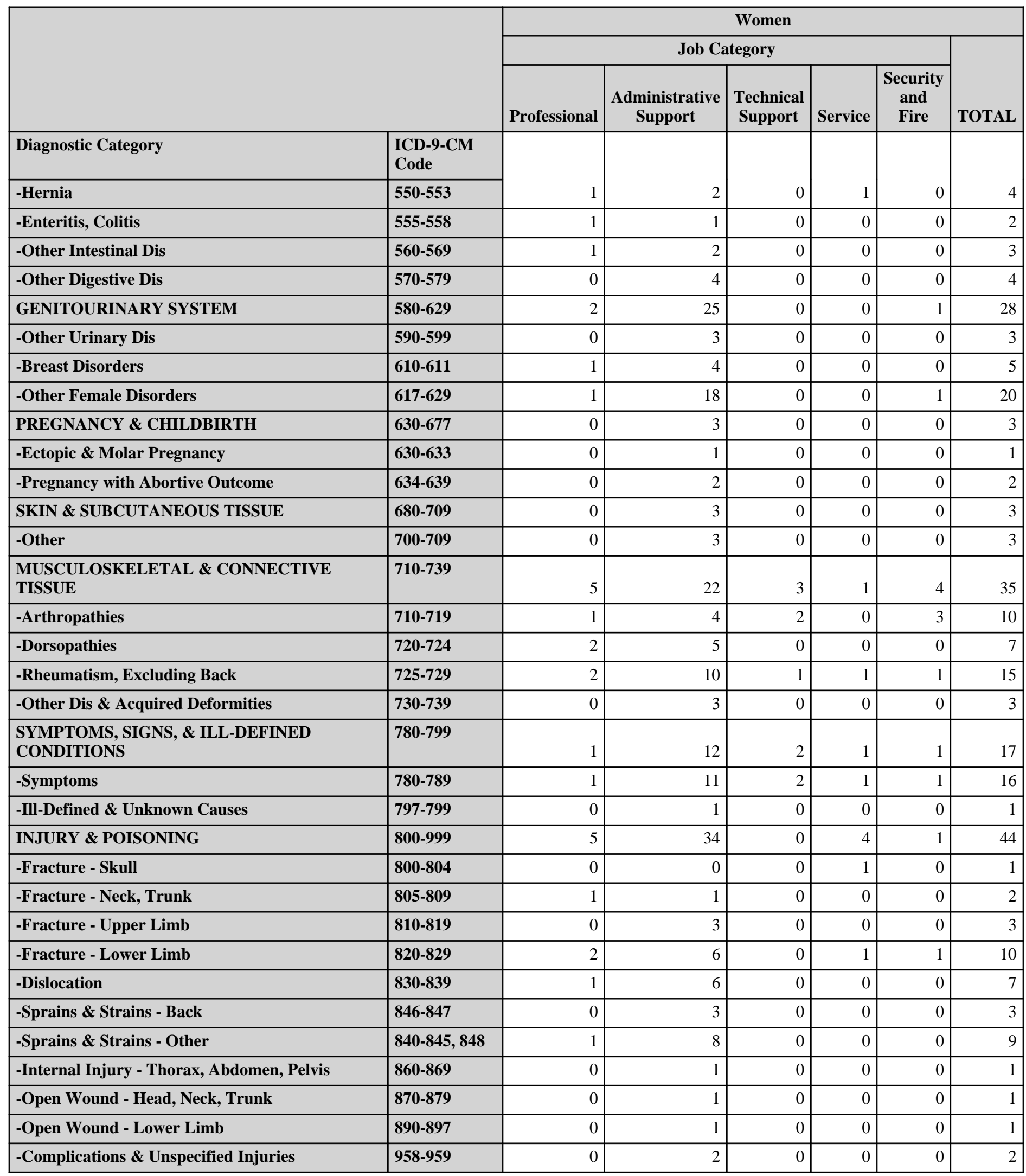

(Continued)

*Only those diagnostic categories and gender/job category combinations with at least one occurrence appear in this table. 


\section{Lawrence Livermore National Laboratory 2007}

Absence Data

Appendix I. Number of Diagnoses in Each Diagnostic Category by Gender and Job Category*

\begin{tabular}{|c|c|c|c|c|c|c|c|}
\hline & & \multicolumn{6}{|c|}{ Women } \\
\hline & & \multicolumn{5}{|c|}{ Job Category } & \multirow[b]{2}{*}{ TOTAL } \\
\hline & & Professional & $\begin{array}{l}\text { Administrative } \\
\text { Support }\end{array}$ & $\begin{array}{l}\text { Technical } \\
\text { Support }\end{array}$ & Service & $\begin{array}{c}\text { Security } \\
\text { and } \\
\text { Fire }\end{array}$ & \\
\hline Diagnostic Category & $\begin{array}{l}\text { ICD-9-CM } \\
\text { Code }\end{array}$ & \multirow[b]{2}{*}{0} & \multirow[b]{2}{*}{2} & \multirow[b]{2}{*}{0} & \multirow[b]{2}{*}{2} & \multirow[b]{2}{*}{0} & \multirow[b]{2}{*}{4} \\
\hline -Complications of Surgical/Medical Care & 996-999 & & & & & & \\
\hline $\begin{array}{l}\text { HEALTH STATUS/HEALTH SERVICE } \\
\text { CONTACT }\end{array}$ & V01-V82 & 1 & 5 & 0 & 0 & 0 & 6 \\
\hline -Specific Procedures/Aftercare & V50-V59 & 1 & 5 & 0 & 0 & 0 & 6 \\
\hline
\end{tabular}

\begin{tabular}{|c|c|c|c|c|c|c|}
\hline & \multicolumn{6}{|c|}{ Women } \\
\hline & \multicolumn{5}{|c|}{ Job Category } & \multirow[b]{2}{*}{ TOTAL } \\
\hline & Professional & $\begin{array}{c}\text { Administrative } \\
\text { Support }\end{array}$ & $\begin{array}{r}\text { Technica } \\
\text { Support }\end{array}$ & Service & $\begin{array}{c}\text { Security } \\
\text { and } \\
\text { Fire }\end{array}$ & \\
\hline $\begin{array}{l}\text { Diagnostic } \\
\text { Category }\end{array}$ & \multirow[b]{2}{*}{33} & \multirow[b]{2}{*}{195} & \multirow[b]{2}{*}{11} & \multirow[b]{2}{*}{11} & \multirow[b]{2}{*}{11} & \multirow[b]{2}{*}{261} \\
\hline Total & & & & & & \\
\hline
\end{tabular}

*Only those diagnostic categories and gender/job category combinations with at least one occurrence appear in this table. 


\section{Lawrence Livermore National Laboratory 2007}

\section{Absence Data}

Appendix I. Number of Diagnoses in Each Diagnostic Category by Gender and Job Category*

\begin{tabular}{|c|c|c|c|c|c|c|c|c|}
\hline & & \multicolumn{7}{|c|}{ Men } \\
\hline & & \multicolumn{6}{|c|}{ Job Category } & \multirow[b]{2}{*}{ TOTAL } \\
\hline & & Professional & $\begin{array}{l}\text { Administrative } \\
\text { Support }\end{array}$ & $\begin{array}{l}\text { Technical } \\
\text { Support }\end{array}$ & Service & $\begin{array}{c}\text { Security } \\
\text { and } \\
\text { Fire }\end{array}$ & Crafts & \\
\hline Diagnostic Category & $\begin{array}{l}\text { ICD-9-CM } \\
\text { Code }\end{array}$ & & & & & & & \\
\hline $\begin{array}{l}\text { INFECTIOUS \& PARASITIC DISEASES } \\
\text { (DIS) }\end{array}$ & 001-139 & 16 & 6 & 4 & 4 & 3 & 2 & 35 \\
\hline -Intestinal Infectious Dis & 001-009 & 3 & 1 & 1 & 1 & 1 & 0 & 7 \\
\hline -Other Bacterial Dis & 030-041 & 4 & 2 & 0 & 1 & 0 & 1 & 8 \\
\hline -Viral Dis with Exanthem & 050-057 & 3 & 0 & 0 & 0 & 0 & 0 & 3 \\
\hline -Other Viral Dis \& Chlamydiae & 070-079 & 6 & 2 & 3 & 1 & 2 & 1 & 15 \\
\hline -Other Infections \& Parasitic Dis & $130-136$ & 0 & 1 & 0 & 1 & 0 & 0 & 2 \\
\hline MALIGNANT NEOPLASMS & $\begin{array}{l}\text { 140-208, } \\
230-234\end{array}$ & 6 & 6 & 2 & 1 & 1 & 0 & 16 \\
\hline -Digestive \& Peritoneal & $150-159$ & 0 & 2 & 0 & 0 & 0 & 0 & 2 \\
\hline -Bone, Connective Tissue, Skin & $\begin{array}{l}170-173, \\
176\end{array}$ & 2 & 1 & 0 & 0 & 0 & 0 & 3 \\
\hline -Genitourinary & 179-189 & 4 & 2 & 2 & 1 & 1 & 0 & 10 \\
\hline -Lymphatic \& Hematopoietic & $200-208$ & 0 & 1 & 0 & 0 & 0 & 0 & 1 \\
\hline BENIGN \& UNCERTAIN NEOPLASMS & $\begin{array}{l}\text { 210-229, } \\
235-239\end{array}$ & 2 & 4 & 1 & 0 & 0 & 0 & 7 \\
\hline ENDOCRINE/METABOLIC/IMMUNITY & $240-279$ & 2 & 2 & 1 & 0 & 0 & 1 & 6 \\
\hline -Thyroid Gland Disorders & $240-246$ & 0 & 1 & 0 & 0 & 0 & 0 & 1 \\
\hline -Other Endocrine Gland Dis & 250-259 & 1 & 0 & 0 & 0 & 0 & 1 & 2 \\
\hline -Other Metabolic \& Immunity Disorders & 270-279 & 1 & 1 & 1 & 0 & 0 & 0 & 3 \\
\hline BLOOD \& BLOOD-FORMING ORGANS & $280-289$ & 0 & 0 & 0 & 0 & 0 & 1 & 1 \\
\hline MENTAL DISORDERS & 290-319 & 1 & 1 & 0 & 1 & 0 & 0 & 3 \\
\hline -Non-Psychotic Disorders & $\begin{array}{l}\text { 300-302, } \\
306-316\end{array}$ & 1 & 1 & 0 & 1 & 0 & 0 & 3 \\
\hline $\begin{array}{l}\text { NERVOUS SYSTEM (NS) \& SENSE } \\
\text { ORGANS }\end{array}$ & 320-389 & 9 & 8 & 3 & 2 & 0 & 1 & 23 \\
\hline -Hereditary/Degenerative Central NS Dis & 330-337 & 0 & 1 & 0 & 0 & 0 & 0 & 1 \\
\hline -Other Disorders of Central NS & $340-349$ & 0 & 1 & 0 & 0 & 0 & 0 & 1 \\
\hline -Disorders of Peripheral NS & $350-359$ & 0 & 4 & 1 & 0 & 0 & 0 & 5 \\
\hline -Disorders of Eye & $360-379$ & 9 & 1 & 1 & 2 & 0 & 0 & 13 \\
\hline -Dis of Ear \& Mastoid & $380-389$ & 0 & 1 & 1 & 0 & 0 & 1 & 3 \\
\hline CIRCULATORY SYSTEM & $390-459$ & 13 & 8 & 2 & 1 & 1 & 1 & 26 \\
\hline -Chronic Rheumatic Heart Dis & 393-398 & 0 & 0 & 0 & 1 & 0 & 0 & 1 \\
\hline -Hypertensive Dis & 401-405 & 1 & 0 & 0 & 0 & 0 & 0 & 1 \\
\hline -Ischemic Heart Dis & $410-414$ & 3 & 3 & 1 & 0 & 0 & 0 & 7 \\
\hline -Other Heart Dis & $420-429$ & 5 & 1 & 0 & 0 & 1 & 0 & 7 \\
\hline -Cerebrovascular Dis & $430-438$ & 1 & 0 & 0 & 0 & 0 & 0 & 1 \\
\hline
\end{tabular}

(Continued)

*Only those diagnostic categories and gender/job category combinations with at least one occurrence appear in this table. 


\section{Lawrence Livermore National Laboratory 2007}

\section{Absence Data}

Appendix I. Number of Diagnoses in Each Diagnostic Category by Gender and Job Category*

\begin{tabular}{|c|c|c|c|c|c|c|c|c|}
\hline & & \multicolumn{7}{|c|}{ Men } \\
\hline & & \multicolumn{6}{|c|}{ Job Category } & \multirow[b]{2}{*}{ TOTAL } \\
\hline & & Professional & $\begin{array}{l}\text { Administrative } \\
\text { Support }\end{array}$ & $\begin{array}{l}\text { Technical } \\
\text { Support }\end{array}$ & Service & $\begin{array}{c}\text { Security } \\
\text { and } \\
\text { Fire }\end{array}$ & Crafts & \\
\hline Diagnostic Category & $\begin{array}{l}\text { ICD-9-CM } \\
\text { Code }\end{array}$ & & & & & & & \\
\hline -Dis of Arteries \& Capillaries & $440-448$ & 1 & 1 & 0 & 0 & 0 & 0 & 2 \\
\hline -Dis of Veins, Lymphatics, Other & $451-459$ & 2 & 3 & 1 & 0 & 0 & 1 & 7 \\
\hline RESPIRATORY SYSTEM & $460-519$ & 30 & 22 & 12 & 11 & 4 & 3 & 82 \\
\hline -Acute Respiratory Infections & $460-466$ & 13 & 6 & 6 & 4 & 2 & 0 & 31 \\
\hline -Other Dis Upper Respiratory Tract & $470-478$ & 5 & 4 & 2 & 1 & 0 & 0 & 12 \\
\hline -Pneumonia \& Influenza & 480-487 & 8 & 8 & 3 & 4 & 0 & 3 & 26 \\
\hline -Chronic Obstructive Dis & $490-496$ & 2 & 2 & 0 & 2 & 2 & 0 & 8 \\
\hline -Other Respiratory Dis & $510-519$ & 2 & 2 & 1 & 0 & 0 & 0 & 5 \\
\hline DIGESTIVE SYSTEM & $520-579$ & 17 & 15 & 12 & 8 & 0 & 3 & 55 \\
\hline -Oral Cavity, Saliva Glands, Jaw & $520-529$ & 2 & 1 & 1 & 0 & 0 & 1 & 5 \\
\hline -Esophagus, Stomach, Duodenum & $530-537$ & 0 & 3 & 1 & 0 & 0 & 0 & 4 \\
\hline -Appendicitis & $540-543$ & 0 & 0 & 0 & 1 & 0 & 1 & 2 \\
\hline -Hernia & $550-553$ & 7 & 7 & 3 & 4 & 0 & 1 & 22 \\
\hline -Enteritis, Colitis & $555-558$ & 3 & 1 & 1 & 1 & 0 & 0 & $\overline{6}$ \\
\hline -Other Intestinal Dis & $560-569$ & 1 & 2 & 3 & 1 & 0 & 0 & 7 \\
\hline -Other Digestive Dis & $570-579$ & 4 & 1 & 3 & 1 & 0 & 0 & 9 \\
\hline GENITOURINARY SYSTEM & $580-629$ & 3 & 5 & 1 & 0 & 0 & 1 & 10 \\
\hline -Nephritis, Nephrosis & $580-589$ & 0 & 1 & 0 & 0 & 0 & 1 & 2 \\
\hline -Other Urinary Dis & $590-599$ & 0 & 2 & 1 & 0 & 0 & 0 & 3 \\
\hline -Male Genital Organ Dis & $600-608$ & 3 & 1 & 0 & 0 & 0 & 0 & 4 \\
\hline -Breast Disorders & $610-611$ & 0 & 1 & 0 & 0 & 0 & 0 & 1 \\
\hline SKIN \& SUBCUTANEOUS TISSUE & $680-709$ & 1 & 3 & 1 & 0 & 1 & 0 & 6 \\
\hline -Infections & $680-686$ & 1 & 3 & 1 & 0 & 1 & 0 & 6 \\
\hline $\begin{array}{l}\text { MUSCULOSKELETAL \& CONNECTIVE } \\
\text { TISSUE }\end{array}$ & 710-739 & 26 & 14 & 7 & 3 & 7 & 7 & 64 \\
\hline -Arthropathies & $710-719$ & 5 & 6 & 3 & 1 & 0 & 4 & 19 \\
\hline -Dorsopathies & $720-724$ & 14 & 6 & 2 & 0 & 5 & 3 & 30 \\
\hline -Rheumatism, Excluding Back & $725-729$ & 6 & 2 & 1 & 1 & 2 & 0 & 12 \\
\hline -Other Dis \& Acquired Deformities & $730-739$ & 1 & 0 & 1 & 1 & 0 & 0 & 3 \\
\hline $\begin{array}{l}\text { SYMPTOMS, SIGNS, \& ILL-DEFINED } \\
\text { CONDITIONS }\end{array}$ & $780-799$ & 9 & 9 & 7 & 2 & 0 & 1 & 28 \\
\hline -Symptoms & 780-789 & 9 & 9 & 7 & 2 & 0 & 1 & 28 \\
\hline INJURY \& POISONING & \begin{tabular}{|c|}
$800-999$ \\
\end{tabular} & 21 & 15 & 18 & 7 & 9 & 5 & 75 \\
\hline -Fracture - Neck, Trunk & $805-809$ & 1 & 0 & 0 & 0 & 0 & 1 & 2 \\
\hline -Fracture - Upper Limb & 810-819 & 1 & 0 & 2 & 0 & 3 & 0 & 6 \\
\hline
\end{tabular}

(Continued)

*Only those diagnostic categories and gender/job category combinations with at least one occurrence appear in this table. 


\section{Lawrence Livermore National Laboratory 2007}

Absence Data

Appendix I. Number of Diagnoses in Each Diagnostic Category by Gender and Job Category*

\begin{tabular}{|c|c|c|c|c|c|c|c|c|}
\hline & & \multicolumn{7}{|c|}{ Men } \\
\hline & & \multicolumn{6}{|c|}{ Job Category } & \multirow[b]{2}{*}{ TOTAL } \\
\hline & & Professional & $\begin{array}{c}\text { Administrative } \\
\text { Support }\end{array}$ & $\begin{array}{l}\text { Technical } \\
\text { Support }\end{array}$ & Service & $\begin{array}{c}\text { Security } \\
\text { and } \\
\text { Fire }\end{array}$ & Crafts & \\
\hline Diagnostic Category & $\begin{array}{l}\text { ICD-9-CM } \\
\text { Code }\end{array}$ & & & & & & & \\
\hline -Fracture - Lower Limb & $820-829$ & 1 & 3 & 2 & 1 & 0 & 0 & 7 \\
\hline -Dislocation & 830-839 & 1 & 2 & 3 & 4 & 1 & 1 & 12 \\
\hline -Sprains \& Strains - Back & 846-847 & 5 & 0 & 1 & 0 & 3 & 0 & 9 \\
\hline -Sprains \& Strains - Other & $\begin{array}{l}\text { 840-845, } \\
848\end{array}$ & 8 & 4 & 8 & 1 & 1 & 2 & 24 \\
\hline -Open Wound - Upper Limb & $\mathbf{8 8 0 - 8 8 7}$ & 1 & 0 & 0 & 0 & 0 & 0 & 1 \\
\hline -Open Wound - Lower Limb & 890-897 & 0 & 2 & 0 & 0 & 1 & 0 & 3 \\
\hline -Superficial Injury & $910-919$ & 0 & 0 & 0 & 0 & 0 & 1 & 1 \\
\hline -Contusion & $920-924$ & 1 & 0 & 1 & 0 & 0 & 0 & 2 \\
\hline -Burns & $940-949$ & 1 & 1 & 0 & 0 & 0 & 0 & 2 \\
\hline -Complications \& Unspecified Injuries & 958-959 & 1 & 1 & 0 & 1 & 0 & 0 & 3 \\
\hline -Unspecified Effects - External Causes & $990-995$ & 0 & 1 & 1 & 0 & 0 & 0 & 2 \\
\hline -Complications of Surgical/Medical Care & 996-999 & 0 & 1 & 0 & 0 & 0 & 0 & 1 \\
\hline $\begin{array}{l}\text { HEALTH STATUS/HEALTH SERVICE } \\
\text { CONTACT }\end{array}$ & V01-V82 & 3 & 0 & 1 & 0 & 1 & 3 & 8 \\
\hline -Isolation \& Prophylactic Measures & V07-V09 & 0 & 0 & 0 & 0 & 0 & 1 & 1 \\
\hline -Personal \& Family History & V10-V19 & 1 & 0 & 0 & 0 & 0 & 0 & 1 \\
\hline -Health Services Reproduction/Development & V20-V29 & 0 & 0 & 1 & 0 & 0 & 1 & 2 \\
\hline -Health Status & V40-V49 & 1 & 0 & 0 & 0 & 0 & 0 & 1 \\
\hline -Specific Procedures/Aftercare & V50-V59 & 1 & 0 & 0 & 0 & 0 & 1 & 2 \\
\hline -Other Circumstances & V60-V69 & 0 & 0 & 0 & 0 & 1 & 0 & 1 \\
\hline
\end{tabular}

\begin{tabular}{|c|c|c|c|c|c|c|c|}
\hline & \multicolumn{7}{|c|}{ Men } \\
\hline & \multicolumn{6}{|c|}{ Job Category } & \multirow[b]{2}{*}{ TOTAL } \\
\hline & Professional & $\begin{array}{c}\text { Administrative } \\
\text { Support }\end{array}$ & $\begin{array}{c}\text { Technical } \\
\text { Support }\end{array}$ & Service & $\begin{array}{c}\text { Security } \\
\text { and } \\
\text { Fire }\end{array}$ & Crafts & \\
\hline $\begin{array}{l}\text { Diagnostic } \\
\text { Category }\end{array}$ & & & & & & & \\
\hline Total & 159 & 118 & 72 & 40 & 27 & 29 & 445 \\
\hline
\end{tabular}

*Only those diagnostic categories and gender/job category combinations with at least one occurrence appear in this table. 


\section{Lawrence Livermore National Laboratory 2007}

Absence Data

Appendix J. Total Number of Calendar Days Absent in Each Diagnostic Category by Gender and Job Category*

\begin{tabular}{|c|c|c|c|c|c|c|c|}
\hline & & \multicolumn{6}{|c|}{ Women } \\
\hline & & \multicolumn{5}{|c|}{ Job Category } & \multirow[b]{2}{*}{ TOTAL } \\
\hline & & Professional & $\begin{array}{l}\text { Administrative } \\
\text { Support }\end{array}$ & $\begin{array}{l}\text { Technical } \\
\text { Support }\end{array}$ & Service & $\begin{array}{c}\text { Security } \\
\text { and } \\
\text { Fire }\end{array}$ & \\
\hline Diagnostic Category & $\begin{array}{l}\text { ICD-9-CM } \\
\text { Code }\end{array}$ & \multirow[b]{2}{*}{20} & \multirow[b]{2}{*}{29} & \multirow[b]{2}{*}{0} & \multirow[b]{2}{*}{0} & \multirow[b]{2}{*}{0} & \multirow[b]{2}{*}{49} \\
\hline INFECTIOUS \& PARASITIC DISEASES (DIS) & 001-139 & & & & & & \\
\hline MALIGNANT NEOPLASMS & $\begin{array}{l}140-208 \\
230-234\end{array}$ & 0 & 803 & 12 & 0 & 4 & 819 \\
\hline BENIGN \& UNCERTAIN NEOPLASMS & $\begin{array}{l}\text { 210-229, } \\
\text { 235-239 }\end{array}$ & 44 & 462 & 47 & 47 & 0 & 600 \\
\hline ENDOCRINE/METABOLIC/IMMUNITY & $240-279$ & 0 & 132 & 0 & 0 & 0 & 132 \\
\hline BLOOD \& BLOOD-FORMING ORGANS & $280-289$ & 426 & 14 & 0 & 0 & 0 & 440 \\
\hline MENTAL DISORDERS & 290-319 & 0 & 454 & 0 & 0 & 0 & 454 \\
\hline NERVOUS SYSTEM (NS) \& SENSE ORGANS & $320-389$ & 31 & 168 & 0 & 0 & 0 & 199 \\
\hline CIRCULATORY SYSTEM & $390-459$ & 4 & 169 & 26 & 0 & 0 & 199 \\
\hline RESPIRATORY SYSTEM & $460-519$ & 48 & 364 & 34 & 17 & 19 & 482 \\
\hline DIGESTIVE SYSTEM & $520-579$ & 456 & 239 & 22 & 62 & 0 & 779 \\
\hline GENITOURINARY SYSTEM & $580-629$ & 40 & 887 & 0 & 0 & 4 & 931 \\
\hline PREGNANCY \& CHILDBIRTH & $630-677$ & 0 & 67 & 0 & 0 & 0 & 67 \\
\hline SKIN \& SUBCUTANEOUS TISSUE & 680-709 & 0 & 77 & 0 & 0 & 0 & 77 \\
\hline $\begin{array}{l}\text { MUSCULOSKELETAL \& CONNECTIVE } \\
\text { TISSUE }\end{array}$ & $710-739$ & 476 & 1,125 & 283 & 26 & 224 & 2,134 \\
\hline $\begin{array}{l}\text { SYMPTOMS, SIGNS, \& ILL-DEFINED } \\
\text { CONDITIONS }\end{array}$ & $780-799$ & 3 & 193 & 169 & 1 & 15 & 381 \\
\hline INJURY \& POISONING & $800-999$ & 553 & 894 & 0 & 211 & 149 & 1,807 \\
\hline
\end{tabular}

\begin{tabular}{|c|c|c|c|c|c|c|c|c|}
\hline & & \multicolumn{7}{|c|}{ Men } \\
\hline & & \multicolumn{6}{|c|}{ Job Category } & \multirow[b]{2}{*}{ TOTAL } \\
\hline & & Professional & $\begin{array}{c}\text { Administrative } \\
\text { Support }\end{array}$ & $\begin{array}{l}\text { Technical } \\
\text { Support }\end{array}$ & Service & $\begin{array}{c}\text { Security } \\
\text { and } \\
\text { Fire }\end{array}$ & Crafts & \\
\hline Diagnostic Category & $\begin{array}{l}\text { ICD-9-CM } \\
\text { Code }\end{array}$ & & & & & & & \\
\hline $\begin{array}{l}\text { INFECTIOUS \& PARASITIC DISEASES } \\
\text { (DIS) }\end{array}$ & 001-139 & 486 & 65 & 26 & 75 & 25 & 34 & 711 \\
\hline MALIGNANT NEOPLASMS & $\begin{array}{l}\text { 140-208, } \\
230-234\end{array}$ & 271 & 483 & 71 & 60 & 70 & 0 & 955 \\
\hline BENIGN \& UNCERTAIN NEOPLASMS & $\begin{array}{l}210-229, \\
235-239\end{array}$ & 36 & 147 & 12 & 0 & 0 & 0 & 195 \\
\hline ENDOCRINE/METABOLIC/IMMUNITY & 240-279 & 12 & 43 & 5 & 0 & 0 & 136 & 196 \\
\hline BLOOD \& BLOOD-FORMING ORGANS & $280-289$ & 0 & 0 & 0 & 0 & 0 & 66 & 66 \\
\hline MENTAL DISORDERS & $290-319$ & 9 & 43 & 0 & 31 & 0 & 0 & 83 \\
\hline
\end{tabular}

(Continued) 


\section{Lawrence Livermore National Laboratory 2007}

Absence Data

Appendix J. Total Number of Calendar Days Absent in Each Diagnostic Category by Gender and Job Category*

\begin{tabular}{|c|c|c|c|c|c|c|c|c|}
\hline & & \multicolumn{7}{|c|}{ Men } \\
\hline & & \multicolumn{6}{|c|}{ Job Category } & \multirow[b]{2}{*}{ TOTAL } \\
\hline & & Professional & $\begin{array}{l}\text { Administrative } \\
\text { Support }\end{array}$ & $\begin{array}{l}\text { Technical } \\
\text { Support }\end{array}$ & Service & $\begin{array}{c}\text { Security } \\
\text { and } \\
\text { Fire }\end{array}$ & Crafts & \\
\hline Diagnostic Category & $\begin{array}{l}\text { ICD-9-CM } \\
\text { Code }\end{array}$ & & & & & & & \\
\hline $\begin{array}{l}\text { NERVOUS SYSTEM (NS) \& SENSE } \\
\text { ORGANS }\end{array}$ & $320-389$ & 176 & 278 & 156 & 55 & 0 & 1 & 666 \\
\hline CIRCULATORY SYSTEM & $390-459$ & 502 & 112 & 105 & 173 & 27 & 11 & 930 \\
\hline RESPIRATORY SYSTEM & $460-519$ & 235 & 216 & 124 & 94 & 19 & 27 & 715 \\
\hline DIGESTIVE SYSTEM & $520-579$ & 212 & 214 & 403 & 234 & 0 & 33 & 1,096 \\
\hline GENITOURINARY SYSTEM & $580-629$ & 54 & 224 & 7 & 0 & 0 & 11 & 296 \\
\hline SKIN \& SUBCUTANEOUS TISSUE & $680-709$ & 117 & 75 & 15 & 0 & 4 & 0 & 211 \\
\hline $\begin{array}{l}\text { MUSCULOSKELETAL \& CONNECTIVE } \\
\text { TISSUE }\end{array}$ & 710-739 & 498 & 426 & 425 & 53 & 95 & 411 & 1,908 \\
\hline $\begin{array}{l}\text { SYMPTOMS, SIGNS, \& ILL-DEFINED } \\
\text { CONDITIONS }\end{array}$ & $780-799$ & 224 & 80 & 56 & 12 & 0 & 13 & 385 \\
\hline INJURY \& POISONING & $800-999$ & 217 & 588 & 369 & 274 & 212 & 295 & 1,955 \\
\hline
\end{tabular}

\footnotetext{
*Absences with >1 ICD-9-CM code in the same diagnostic category were counted only once. Only those diagnostic categories and gender/job category combinations with at least one occurrence appear in this table.
} 


\section{Lawrence Livermore National Laboratory 2007}

Absence Data

Appendix K. Age-Adjusted Illness and Injury Rates by Diagnostic Category*

Part 1. Men

\begin{tabular}{|c|c|c|c|c|c|}
\hline & & $\begin{array}{c}\text { Number of } \\
\text { Diagnoses }\end{array}$ & $\begin{array}{c}\text { Age-Adjusted } \\
\text { Rate per 1,000** }\end{array}$ & $\begin{array}{c}\text { Lower 95\% } \\
\text { Confidence } \\
\text { Limit per 1,000 }\end{array}$ & \begin{tabular}{|c|} 
Upper $95 \%$ \\
Confidence \\
Limit per 1,000 \\
\end{tabular} \\
\hline Diagnostic Category & ICD-9-CM Code & & & & \\
\hline INFECTIOUS \& PARASITIC DISEASES (DIS) & 001-139 & 35 & 5.7 & 3.9 & 8.4 \\
\hline -Intestinal Infectious Dis & 001-009 & 7 & 1.1 & 0.5 & 2.5 \\
\hline -Other Bacterial Dis & 030-041 & 8 & 1.2 & 0.6 & 2.6 \\
\hline -Viral Dis with Exanthem & 050-057 & 3 & 0.5 & 0.2 & 1.8 \\
\hline -Other Viral Dis \& Chlamydiae & 070-079 & 15 & 2.7 & 1.4 & 4.9 \\
\hline -Other Infections \& Parasitic Dis & $130-136$ & 2 & 0.2 & 0.0 & 0.8 \\
\hline MALIGNANT NEOPLASMS & 140-208, 230-234 & 16 & 1.7 & 1.0 & 2.8 \\
\hline -Digestive \& Peritoneal & 150-159 & 2 & 0.2 & 0.0 & 0.8 \\
\hline -Bone, Connective Tissue, Skin & 170-173, 176 & 3 & 0.3 & 0.1 & 1.0 \\
\hline -Genitourinary & 179-189 & 10 & 1.1 & 0.6 & 2.0 \\
\hline -Lymphatic \& Hematopoietic & $200-208$ & 1 & 0.1 & 0.0 & 0.9 \\
\hline BENIGN \& UNCERTAIN NEOPLASMS & $210-229,235-239$ & 7 & 1.0 & 0.4 & 2.1 \\
\hline ENDOCRINE/METABOLIC/IMMUNITY & $240-279$ & 6 & 0.7 & 0.3 & 1.5 \\
\hline -Thyroid Gland Disorders & $240-246$ & 1 & 0.1 & 0.0 & 0.9 \\
\hline -Other Endocrine Gland Dis & $250-259$ & 2 & 0.2 & 0.0 & 0.8 \\
\hline -Other Metabolic \& Immunity Disorders & $270-279$ & 3 & 0.3 & 0.1 & 1.1 \\
\hline BLOOD \& BLOOD-FORMING ORGANS & $280-289$ & 1 & 0.3 & 0.0 & 2.0 \\
\hline MENTAL DISORDERS & 290-319 & 3 & 0.5 & 0.2 & 1.8 \\
\hline -Non-Psychotic Disorders & $300-302,306-316$ & 3 & 0.5 & 0.2 & 1.8 \\
\hline NERVOUS SYSTEM (NS) \& SENSE ORGANS & 320-389 & 23 & 3.6 & 2.2 & 5.9 \\
\hline -Hereditary/Degenerative Central NS Dis & 330-337 & 1 & 0.1 & 0.0 & 0.9 \\
\hline -Other Disorders of Central NS & 340-349 & 1 & 0.1 & 0.0 & 0.7 \\
\hline -Disorders of Peripheral NS & 350-359 & 5 & 0.7 & 0.3 & 1.9 \\
\hline -Disorders of Eye & $360-379$ & 13 & 2.3 & 1.2 & 4.5 \\
\hline -Dis of Ear \& Mastoid & 380-389 & 3 & 0.3 & 0.1 & 1.1 \\
\hline CIRCULATORY SYSTEM & $390-459$ & 26 & 3.3 & 2.2 & 5.0 \\
\hline -Chronic Rheumatic Heart Dis & 393-398 & 1 & 0.1 & 0.0 & 0.9 \\
\hline -Hypertensive Dis & 401-405 & 1 & 0.1 & 0.0 & 0.7 \\
\hline -Ischemic Heart Dis & $410-414$ & 7 & 1.1 & 0.5 & 2.5 \\
\hline -Other Heart Dis & $420-429$ & 7 & 0.9 & 0.4 & 2.0 \\
\hline -Cerebrovascular Dis & $430-438$ & 1 & 0.1 & 0.0 & 0.9 \\
\hline -Dis of Arteries \& Capillaries & $440-448$ & 2 & 0.2 & 0.1 & 0.9 \\
\hline -Dis of Veins, Lymphatics, Other & 451-459 & 7 & 0.8 & 0.4 & 1.6 \\
\hline RESPIRATORY SYSTEM & $460-519$ & 82 & 13.1 & 10.1 & 16.9 \\
\hline -Acute Respiratory Infections & $460-466$ & 31 & 4.8 & 3.1 & 7.5 \\
\hline -Other Dis Upper Respiratory Tract & $470-478$ & 12 & 1.6 & 0.9 & 3.0 \\
\hline
\end{tabular}

* Only those diagnostic categories with at least one occurrence appear in this table.

**Standardized to age distribution of 2000 U.S. population. 


\section{Lawrence Livermore National Laboratory 2007}

Absence Data

Appendix K. Age-Adjusted Illness and Injury Rates by Diagnostic Category*

Part 1. Men

\begin{tabular}{|c|c|c|c|c|c|}
\hline & & $\begin{array}{l}\text { Number of } \\
\text { Diagnoses }\end{array}$ & $\begin{array}{c}\text { Age-Adjusted } \\
\text { Rate per } 1,000^{* *}\end{array}$ & \begin{tabular}{c|} 
Lower $95 \%$ \\
Confidence \\
Limit per 1,000
\end{tabular} & $\begin{array}{c}\text { Upper } 95 \% \\
\text { Confidence } \\
\text { Limit per 1,000 }\end{array}$ \\
\hline Diagnostic Category & ICD-9-CM Code & & & & \\
\hline -Pneumonia \& Influenza & $480-487$ & 26 & 3.8 & 2.5 & 6.0 \\
\hline -Chronic Obstructive Dis & $490-496$ & 8 & 1.9 & 0.9 & 4.1 \\
\hline -Other Respiratory Dis & $510-519$ & 5 & 0.9 & 0.3 & 2.3 \\
\hline DIGESTIVE SYSTEM & $520-579$ & 55 & 7.6 & 5.7 & 10.1 \\
\hline -Oral Cavity, Saliva Glands, Jaw & 520-529 & 5 & 1.1 & 0.4 & 2.7 \\
\hline -Esophagus, Stomach, Duodenum & 530-537 & 4 & 0.5 & 0.2 & 1.3 \\
\hline -Appendicitis & $540-543$ & 2 & 0.2 & 0.0 & 0.8 \\
\hline -Hernia & $550-553$ & 22 & 2.6 & 1.6 & 4.0 \\
\hline -Enteritis, Colitis & $555-558$ & 6 & 0.8 & 0.3 & 2.0 \\
\hline -Other Intestinal Dis & 560-569 & 7 & 1.1 & 0.5 & 2.5 \\
\hline -Other Digestive Dis & $\begin{array}{l}570-579 \\
\end{array}$ & 9 & 1.4 & 0.7 & 2.8 \\
\hline GENITOURINARY SYSTEM & $580-629$ & 10 & 1.2 & 0.6 & 2.1 \\
\hline -Nephritis, Nephrosis & 580-589 & 2 & 0.2 & 0.1 & 0.9 \\
\hline -Other Urinary Dis & 590-599 & 3 & 0.3 & 0.1 & 1.1 \\
\hline -Male Genital Organ Dis & $600-608$ & 4 & 0.5 & 0.2 & 1.3 \\
\hline -Breast Disorders & 610-611 & 1 & 0.1 & 0.0 & 0.9 \\
\hline SKIN \& SUBCUTANEOUS TISSUE & 680-709 & 6 & 0.8 & 0.3 & 2.0 \\
\hline -Infections & $680-686$ & 6 & 0.8 & 0.3 & 2.0 \\
\hline $\begin{array}{l}\text { MUSCULOSKELETAL \& CONNECTIVE } \\
\text { TISSUE }\end{array}$ & 710-739 & 64 & 8.4 & 6.3 & 11.2 \\
\hline -Arthropathies & 710-719 & 19 & 2.0 & 1.2 & 3.1 \\
\hline -Dorsopathies & 720-724 & 30 & 4.8 & 3.1 & 7.4 \\
\hline -Rheumatism, Excluding Back & 725-729 & 12 & 1.3 & 0.7 & 2.3 \\
\hline -Other Dis \& Acquired Deformities & 730-739 & 3 & 0.3 & 0.1 & 1.1 \\
\hline $\begin{array}{l}\text { SYMPTOMS, SIGNS, \& ILL-DEFINED } \\
\text { CONDITIONS }\end{array}$ & 780-799 & 28 & 3.5 & 2.3 & 5.4 \\
\hline -Symptoms & 780-789 & 28 & 3.5 & 2.3 & 5.4 \\
\hline INJURY \& POISONING & $800-999$ & 75 & 12.0 & 9.2 & 15.7 \\
\hline -Fracture - Skull & 800-804 & 2 & 0.2 & 0.0 & 0.8 \\
\hline -Fracture - Upper Limb & $810-819$ & 6 & 1.3 & 0.6 & 3.1 \\
\hline -Fracture - Lower Limb & $820-829$ & 7 & 1.5 & 0.6 & 3.7 \\
\hline -Dislocation & 830-839 & 12 & 1.8 & 1.0 & 3.4 \\
\hline -Sprains \& Strains - Back & $846-847$ & 9 & 1.4 & 0.6 & 3.2 \\
\hline -Sprains \& Strains - Other & $840-845,848$ & 24 & 3.3 & 2.1 & 5.0 \\
\hline -Open Wound - Head, Neck, Trunk & 870-879 & 4 & 1.1 & 0.3 & 3.5 \\
\hline -Superficial Injury & 910-919 & 1 & 0.1 & 0.0 & 0.7 \\
\hline -Contusion & 920-924 & 2 & 0.2 & 0.1 & 1.0 \\
\hline
\end{tabular}

* Only those diagnostic categories with at least one occurrence appear in this table.

**Standardized to age distribution of 2000 U.S. population. 
Lawrence Livermore National Laboratory 2007

Absence Data

Appendix K. Age-Adjusted Illness and Injury Rates by Diagnostic Category*

Part 1. Men

\begin{tabular}{|c|c|c|c|c|c|}
\hline & & $\begin{array}{l}\text { Number of } \\
\text { Diagnoses }\end{array}$ & $\begin{array}{c}\text { Age-Adjusted } \\
\text { Rate per } 1,000 * *\end{array}$ & $\begin{array}{c}\text { Lower } 95 \% \\
\text { Confidence } \\
\text { Limit per 1,000 }\end{array}$ & $\begin{array}{c}\text { Upper } 95 \% \\
\text { Confidence } \\
\text { Limit per 1,000 }\end{array}$ \\
\hline Diagnostic Category & ICD-9-CM Code & \multirow[b]{2}{*}{2} & \multirow[b]{2}{*}{0.2} & \multirow[b]{2}{*}{0.0} & \multirow[b]{2}{*}{0.8} \\
\hline -Burns & $940-949$ & & & & \\
\hline -Complications \& Unspecified Injuries & 958-959 & 3 & 0.6 & 0.2 & 2.2 \\
\hline -Unspecified Effects - External Causes & 990-995 & 2 & 0.2 & 0.1 & 0.9 \\
\hline -Complications of Surgical/Medical Care & 996-999 & 1 & 0.1 & 0.0 & 0.7 \\
\hline Total & & 437 & 63.3 & 56.7 & 70.6 \\
\hline
\end{tabular}

*Only those diagnostic categories with at least one occurrence appear in this table.

**Standardized to age distribution of 2000 U.S. population. 


\section{Lawrence Livermore National Laboratory 2007}

Absence Data

Appendix K. Age-Adjusted Illness and Injury Rates by Diagnostic Category*

Part 2. Women

\begin{tabular}{|c|c|c|c|c|c|}
\hline & & $\begin{array}{c}\text { Number of } \\
\text { Diagnoses }\end{array}$ & $\begin{array}{l}\text { Age-Adjusted } \\
\text { Rate per 1,000** }\end{array}$ & $\begin{array}{c}\text { Lower 95\% } \\
\text { Confidence } \\
\text { Limit per 1,000 }\end{array}$ & \begin{tabular}{|c|} 
Upper $95 \%$ \\
Confidence \\
Limit per 1,000
\end{tabular} \\
\hline Diagnostic Category & ICD-9-CM Code & & & & \\
\hline INFECTIOUS \& PARASITIC DISEASES (DIS) & 001-139 & 9 & 3.6 & 1.7 & 7.6 \\
\hline -Intestinal Infectious Dis & 001-009 & 3 & 0.7 & 0.2 & 2.2 \\
\hline -Other Bacterial Dis & 030-041 & 2 & 1.4 & 0.3 & 5.6 \\
\hline -Viral Dis with Exanthem & 050-057 & 1 & 0.2 & 0.0 & 1.6 \\
\hline -Other Viral Dis \& Chlamydiae & 070-079 & 3 & 1.3 & 0.3 & 4.8 \\
\hline MALIGNANT NEOPLASMS & $140-208,230-234$ & 16 & 4.4 & 2.6 & 7.4 \\
\hline -Breast & 174-175 & 12 & 3.4 & 1.9 & 6.3 \\
\hline -Genitourinary & 179-189 & 4 & 0.9 & 0.3 & 2.5 \\
\hline BENIGN \& UNCERTAIN NEOPLASMS & 210-229, 235-239 & 13 & 4.7 & 2.6 & 8.5 \\
\hline ENDOCRINE/METABOLIC/IMMUNITY & $240-279$ & 3 & 1.0 & 0.3 & 3.5 \\
\hline -Other Metabolic \& Immunity Disorders & $270-279$ & 3 & 1.0 & 0.3 & 3.5 \\
\hline BLOOD \& BLOOD-FORMING ORGANS & $280-289$ & 2 & 0.5 & 0.1 & 1.9 \\
\hline MENTAL DISORDERS & $290-319$ & 5 & 2.2 & 0.9 & 5.6 \\
\hline -Non-Psychotic Disorders & $300-302,306-316$ & 5 & 2.2 & 0.9 & 5.6 \\
\hline NERVOUS SYSTEM (NS) \& SENSE ORGANS & 320-389 & 18 & 5.7 & 3.3 & 9.7 \\
\hline -Disorders of Peripheral NS & 350-359 & 4 & 1.3 & 0.4 & 3.7 \\
\hline -Disorders of Eye & $360-379$ & 6 & 1.4 & 0.6 & 3.1 \\
\hline -Dis of Ear \& Mastoid & 380-389 & 8 & 3.0 & 1.3 & 6.8 \\
\hline CIRCULATORY SYSTEM & $390-459$ & 7 & 2.0 & 0.9 & 4.3 \\
\hline -Hypertensive Dis & $401-405$ & 1 & 0.2 & 0.0 & 1.6 \\
\hline -Ischemic Heart Dis & $410-414$ & 1 & 0.5 & 0.1 & 3.9 \\
\hline -Cerebrovascular Dis & $430-438$ & 1 & 0.2 & 0.0 & 1.6 \\
\hline -Dis of Arteries \& Capillaries & $440-448$ & 3 & 0.7 & 0.2 & 2.2 \\
\hline -Dis of Veins, Lymphatics, Other & 451-459 & 1 & 0.2 & 0.0 & 1.7 \\
\hline RESPIRATORY SYSTEM & $460-519$ & 37 & 12.5 & 8.7 & 17.8 \\
\hline -Acute Respiratory Infections & $460-466$ & 19 & 5.8 & 3.5 & 9.5 \\
\hline -Other Dis Upper Respiratory Tract & $470-478$ & 4 & 1.6 & 0.6 & 4.6 \\
\hline -Pneumonia \& Influenza & $480-487$ & 10 & 3.9 & 1.9 & 7.7 \\
\hline -Chronic Obstructive Dis & $490-496$ & 3 & 1.0 & 0.3 & 3.5 \\
\hline -Other Respiratory Dis & $510-519$ & 1 & 0.2 & 0.0 & 1.6 \\
\hline DIGESTIVE SYSTEM & $520-579$ & 15 & 4.5 & 2.6 & 7.8 \\
\hline -Oral Cavity, Saliva Glands, Jaw & $520-529$ & 1 & 0.2 & 0.0 & 1.6 \\
\hline -Appendicitis & $540-543$ & 1 & 0.2 & 0.0 & 1.6 \\
\hline -Hernia & $550-553$ & 4 & 0.9 & 0.4 & 2.5 \\
\hline -Enteritis, Colitis & $555-558$ & 2 & 0.8 & 0.2 & 3.5 \\
\hline -Other Intestinal Dis & $560-569$ & 3 & 0.7 & 0.2 & 2.1 \\
\hline
\end{tabular}

* Only those diagnostic categories with at least one occurrence appear in this table.

**Standardized to age distribution of 2000 U.S. population. 


\section{Lawrence Livermore National Laboratory 2007}

Absence Data

Appendix K. Age-Adjusted IIIness and Injury Rates by Diagnostic Category*

Part 2. Women

\begin{tabular}{|c|c|c|c|c|c|}
\hline & & $\begin{array}{c}\text { Number of } \\
\text { Diagnoses }\end{array}$ & $\begin{array}{c}\text { Age-Adjusted } \\
\text { Rate per } 1,000 * *\end{array}$ & \begin{tabular}{|c|} 
Lower $95 \%$ \\
Confidence \\
Limit per 1,000
\end{tabular} & $\begin{array}{c}\text { Upper } 95 \% \\
\text { Confidence } \\
\text { Limit per 1,000 }\end{array}$ \\
\hline Diagnostic Category & ICD-9-CM Code & & & & \\
\hline -Other Digestive Dis & 570-579 & 4 & 1.6 & 0.6 & 4.7 \\
\hline GENITOURINARY SYSTEM & $580-629$ & 28 & 9.1 & 6.0 & 13.7 \\
\hline -Other Urinary Dis & 590-599 & 3 & 1.6 & 0.5 & 5.4 \\
\hline -Breast Disorders & $610-611$ & 5 & 1.8 & 0.7 & 4.8 \\
\hline -Other Female Disorders & 617-629 & 20 & 5.7 & 3.5 & 9.1 \\
\hline PREGNANCY \& CHILDBIRTH & $630-677$ & 3 & 1.9 & 0.6 & 6.1 \\
\hline -Ectopic \& Molar Pregnancy & $630-633$ & 1 & 0.8 & 0.1 & 5.6 \\
\hline -Pregnancy with Abortive Outcome & 634-639 & 2 & 1.1 & 0.3 & 4.6 \\
\hline SKIN \& SUBCUTANEOUS TISSUE & 680-709 & 3 & 0.7 & 0.2 & 2.2 \\
\hline -Other & 700-709 & 3 & 0.7 & 0.2 & 2.2 \\
\hline $\begin{array}{l}\text { MUSCULOSKELETAL \& CONNECTIVE } \\
\text { TISSUE }\end{array}$ & 710-739 & 35 & 10.4 & 7.3 & 15.0 \\
\hline -Arthropathies & 710-719 & 10 & 3.0 & 1.5 & 5.8 \\
\hline -Dorsopathies & 720-724 & 7 & 2.3 & 1.0 & 5.2 \\
\hline -Rheumatism, Excluding Back & 725-729 & 15 & 4.4 & 2.6 & 7.7 \\
\hline -Other Dis \& Acquired Deformities & 730-739 & 3 & 0.7 & 0.2 & 2.2 \\
\hline $\begin{array}{l}\text { SYMPTOMS, SIGNS, \& ILL-DEFINED } \\
\text { CONDITIONS }\end{array}$ & 780-799 & 17 & 5.3 & 3.1 & 8.8 \\
\hline -Symptoms & 780-789 & 16 & 5.0 & 2.9 & 8.6 \\
\hline -Ill-Defined \& Unknown Causes & 797-799 & 1 & 0.2 & 0.0 & 1.7 \\
\hline INJURY \& POISONING & $800-999$ & 44 & 15.0 & 10.7 & 20.8 \\
\hline -Fracture - Skull & $800-804$ & 3 & 1.3 & 0.4 & 4.4 \\
\hline -Fracture - Upper Limb & $810-819$ & 3 & 0.7 & 0.2 & 2.2 \\
\hline -Fracture - Lower Limb & $820-829$ & 10 & 3.9 & 2.0 & 7.7 \\
\hline -Dislocation & 830-839 & 7 & 2.3 & 1.0 & 5.1 \\
\hline -Sprains \& Strains - Back & 846-847 & 3 & 1.0 & 0.3 & 3.6 \\
\hline -Sprains \& Strains - Other & $840-845,848$ & 9 & 2.4 & 1.2 & 4.9 \\
\hline -Internal Injury - Thorax, Abdomen, Pelvis & $860-869$ & 1 & 0.2 & 0.0 & 1.7 \\
\hline -Open Wound - Head, Neck, Trunk & 870-879 & 2 & 1.0 & 0.2 & 5.0 \\
\hline -Complications \& Unspecified Injuries & 958-959 & 2 & 0.5 & 0.1 & 1.9 \\
\hline -Complications of Surgical/Medical Care & 996-999 & 4 & 1.5 & 0.5 & 4.8 \\
\hline Total & & 255 & 83.2 & 72.5 & 95.4 \\
\hline
\end{tabular}

* Only those diagnostic categories with at least one occurrence appear in this table.

**Standardized to age distribution of 2000 U.S. population. 


\section{Lawrence Livermore National Laboratory 2007}

Absence Data

Appendix K. Age-Adjusted Illness and Injury Rates by Diagnostic Category*

Part 3. Men and Women

\begin{tabular}{|c|c|c|c|c|c|}
\hline & & $\begin{array}{c}\text { Number of } \\
\text { Diagnoses }\end{array}$ & $\begin{array}{l}\text { Age-Adjusted } \\
\text { Rate per 1,000** }\end{array}$ & \begin{tabular}{|c|} 
Lower $95 \%$ \\
Confidence \\
Limit per 1,000
\end{tabular} & \begin{tabular}{|c|} 
Upper $95 \%$ \\
Confidence \\
Limit per 1,000
\end{tabular} \\
\hline Diagnostic Category & ICD-9-CM Code & & & & \\
\hline INFECTIOUS \& PARASITIC DISEASES (DIS) & 001-139 & 44 & 5.1 & 3.6 & 7.2 \\
\hline -Intestinal Infectious Dis & 001-009 & 10 & 1.0 & 0.5 & 2.0 \\
\hline -Other Bacterial Dis & 030-041 & 10 & 1.3 & 0.6 & 2.6 \\
\hline -Viral Dis with Exanthem & 050-057 & 4 & 0.4 & 0.1 & 1.2 \\
\hline -Other Viral Dis \& Chlamydiae & 070-079 & 18 & 2.2 & 1.3 & 3.8 \\
\hline -Other Infections \& Parasitic Dis & 130-136 & 2 & 0.1 & 0.0 & 0.5 \\
\hline MALIGNANT NEOPLASMS & $140-208,230-234$ & 32 & 2.5 & 1.8 & 3.6 \\
\hline -Digestive \& Peritoneal & $150-159$ & 2 & 0.1 & 0.0 & 0.5 \\
\hline -Bone, Connective Tissue, Skin & 170-173, 176 & 3 & 0.2 & 0.1 & 0.8 \\
\hline -Breast & 174-175 & 12 & 0.9 & 0.5 & 1.7 \\
\hline -Genitourinary & 179-189 & 14 & 1.1 & 0.7 & 1.9 \\
\hline -Lymphatic \& Hematopoietic & $200-208$ & 1 & 0.1 & 0.0 & 0.6 \\
\hline BENIGN \& UNCERTAIN NEOPLASMS & 210-229, 235-239 & 20 & 2.1 & 1.3 & 3.5 \\
\hline ENDOCRINE/METABOLIC/IMMUNITY & $240-279$ & 9 & 0.7 & 0.4 & 1.4 \\
\hline -Thyroid Gland Disorders & $240-246$ & 1 & 0.1 & 0.0 & 0.6 \\
\hline -Other Endocrine Gland Dis & $250-259$ & 2 & 0.1 & 0.0 & 0.5 \\
\hline -Other Metabolic \& Immunity Disorders & $270-279$ & 6 & 0.5 & 0.2 & 1.1 \\
\hline BLOOD \& BLOOD-FORMING ORGANS & $280-289$ & 3 & 0.3 & 0.1 & 1.2 \\
\hline MENTAL DISORDERS & $290-319$ & 8 & 1.1 & 0.5 & 2.3 \\
\hline -Non-Psychotic Disorders & $300-302,306-316$ & 8 & 1.1 & 0.5 & 2.3 \\
\hline NERVOUS SYSTEM (NS) \& SENSE ORGANS & $320-389$ & 41 & 4.3 & 3.0 & 6.2 \\
\hline -Hereditary/Degenerative Central NS Dis & 330-337 & 1 & 0.1 & 0.0 & 0.6 \\
\hline -Other Disorders of Central NS & $340-349$ & 1 & 0.1 & 0.0 & 0.5 \\
\hline -Disorders of Peripheral NS & 350-359 & 9 & 0.8 & 0.4 & 1.6 \\
\hline -Disorders of Eye & $360-379$ & 19 & 2.1 & 1.2 & 3.5 \\
\hline -Dis of Ear \& Mastoid & 380-389 & 11 & 1.3 & 0.6 & 2.6 \\
\hline CIRCULATORY SYSTEM & $390-459$ & 33 & 2.9 & 2.0 & 4.2 \\
\hline -Chronic Rheumatic Heart Dis & 393-398 & 1 & 0.1 & 0.0 & 0.6 \\
\hline -Hypertensive Dis & $401-405$ & 2 & 0.1 & 0.0 & 0.5 \\
\hline -Ischemic Heart Dis & $410-414$ & 8 & 0.9 & 0.4 & 1.9 \\
\hline -Other Heart Dis & $420-429$ & 7 & 0.6 & 0.3 & 1.4 \\
\hline -Cerebrovascular Dis & $430-438$ & 2 & 0.2 & 0.0 & 0.7 \\
\hline -Dis of Arteries \& Capillaries & $440-448$ & 5 & 0.4 & 0.2 & 1.0 \\
\hline -Dis of Veins, Lymphatics, Other & 451-459 & 8 & 0.6 & 0.3 & 1.3 \\
\hline RESPIRATORY SYSTEM & $460-519$ & 119 & 12.7 & 10.3 & 15.5 \\
\hline -Acute Respiratory Infections & $460-466$ & 50 & 5.2 & 3.8 & 7.2 \\
\hline
\end{tabular}

* Only those diagnostic categories with at least one occurrence appear in this table.

**Standardized to age distribution of 2000 U.S. population. 


\section{Lawrence Livermore National Laboratory 2007}

Absence Data

Appendix K. Age-Adjusted Illness and Injury Rates by Diagnostic Category*

Part 3. Men and Women

\begin{tabular}{|c|c|c|c|c|c|}
\hline & & $\begin{array}{c}\text { Number of } \\
\text { Diagnoses }\end{array}$ & \begin{tabular}{|c|} 
Age-Adjusted \\
Rate per 1,000***
\end{tabular} & $\begin{array}{c}\text { Lower 95\% } \\
\text { Confidence } \\
\text { Limit per 1,000 }\end{array}$ & \begin{tabular}{|c|} 
Upper $95 \%$ \\
Confidence \\
Limit per 1,000
\end{tabular} \\
\hline Diagnostic Category & ICD-9-CM Code & & & & \\
\hline -Other Dis Upper Respiratory Tract & $470-478$ & 16 & 1.5 & 0.9 & 2.6 \\
\hline -Pneumonia \& Influenza & $480-487$ & 36 & 3.7 & 2.6 & $\overline{5.4}$ \\
\hline -Chronic Obstructive Dis & $490-496$ & 11 & 1.5 & 0.8 & 2.9 \\
\hline -Other Respiratory Dis & $510-519$ & 6 & 0.7 & 0.3 & 1.6 \\
\hline DIGESTIVE SYSTEM & 520-579 & 70 & 6.6 & 5.1 & 8.5 \\
\hline -Oral Cavity, Saliva Glands, Jaw & $520-529$ & 6 & 0.8 & 0.3 & 1.9 \\
\hline -Esophagus, Stomach, Duodenum & $530-537$ & 4 & 0.3 & 0.1 & 0.8 \\
\hline -Appendicitis & $540-543$ & 3 & 0.2 & 0.1 & 0.6 \\
\hline -Hernia & $550-553$ & 26 & 2.1 & 1.4 & 3.2 \\
\hline -Enteritis, Colitis & $555-558$ & 8 & 0.8 & 0.4 & 1.6 \\
\hline -Other Intestinal Dis & $560-569$ & 10 & 0.9 & 0.5 & 1.9 \\
\hline -Other Digestive Dis & 570-579 & 13 & 1.4 & 0.8 & 2.6 \\
\hline GENITOURINARY SYSTEM & 580-629 & 38 & 3.6 & 2.5 & 5.2 \\
\hline -Nephritis, Nephrosis & 580-589 & 2 & 0.1 & 0.0 & 0.6 \\
\hline -Other Urinary Dis & 590-599 & 6 & 0.7 & 0.3 & 1.9 \\
\hline -Male Genital Organ Dis & $600-608$ & 4 & 0.3 & 0.1 & 0.9 \\
\hline -Breast Disorders & 610-611 & 6 & 0.7 & 0.3 & 1.6 \\
\hline -Other Female Disorders & $617-629$ & 20 & 1.7 & 1.1 & 2.8 \\
\hline PREGNANCY \& CHILDBIRTH & 630-677 & 3 & 0.7 & 0.2 & 2.1 \\
\hline -Ectopic \& Molar Pregnancy & $630-633$ & 1 & 0.3 & 0.0 & 2.0 \\
\hline -Pregnancy with Abortive Outcome & 634-639 & 2 & 0.4 & 0.1 & 1.5 \\
\hline SKIN \& SUBCUTANEOUS TISSUE & $680-709$ & 9 & 0.8 & 0.4 & 1.7 \\
\hline -Infections & $680-686$ & 6 & 0.6 & 0.3 & 1.5 \\
\hline -Other & 700-709 & 3 & 0.2 & 0.1 & 0.7 \\
\hline $\begin{array}{l}\text { MUSCULOSKELETAL \& CONNECTIVE } \\
\text { TISSUE }\end{array}$ & 710-739 & 99 & 8.8 & 7.1 & 10.9 \\
\hline -Arthropathies & $710-719$ & 29 & 2.2 & 1.5 & 3.2 \\
\hline -Dorsopathies & $720-724$ & 37 & 3.9 & 2.7 & 5.7 \\
\hline -Rheumatism, Excluding Back & $725-729$ & 27 & 2.2 & 1.5 & 3.2 \\
\hline -Other Dis \& Acquired Deformities & 730-739 & 6 & 0.5 & 0.2 & 1.1 \\
\hline $\begin{array}{l}\text { SYMPTOMS, SIGNS, \& ILL-DEFINED } \\
\text { CONDITIONS }\end{array}$ & 780-799 & 45 & 3.9 & 2.8 & 5.3 \\
\hline -Symptoms & 780-789 & 44 & 3.8 & 2.8 & 5.2 \\
\hline -IIl-Defined \& Unknown Causes & 797-799 & 1 & 0.1 & 0.0 & 0.6 \\
\hline INJURY \& POISONING & $800-999$ & 119 & 12.6 & 10.2 & 15.4 \\
\hline -Fracture - Skull & $800-804$ & 5 & 0.4 & 0.2 & 1.0 \\
\hline -Fracture - Upper Limb & $810-819$ & 9 & 1.1 & 0.5 & 2.3 \\
\hline
\end{tabular}

* Only those diagnostic categories with at least one occurrence appear in this table.

**Standardized to age distribution of 2000 U.S. population. 


\section{Lawrence Livermore National Laboratory 2007}

Absence Data

Appendix K. Age-Adjusted Illness and Injury Rates by Diagnostic Category*

Part 3. Men and Women

\begin{tabular}{|c|c|c|c|c|c|}
\hline & & $\begin{array}{l}\text { Number of } \\
\text { Diagnoses }\end{array}$ & $\begin{array}{c}\text { Age-Adjusted } \\
\text { Rate per 1,000** }\end{array}$ & $\begin{array}{c}\text { Lower } 95 \% \\
\text { Confidence } \\
\text { Limit per 1,000 }\end{array}$ & $\begin{array}{c}\text { Upper } 95 \% \\
\text { Confidence } \\
\text { Limit per 1,000 }\end{array}$ \\
\hline Diagnostic Category & ICD-9-CM Code & \multirow[b]{2}{*}{17} & \multirow[b]{2}{*}{2.0} & \multirow[b]{2}{*}{1.1} & \multirow[b]{2}{*}{3.4} \\
\hline -Fracture - Lower Limb & $820-829$ & & & & \\
\hline -Dislocation & $830-839$ & 19 & 1.9 & 1.1 & 3.0 \\
\hline -Sprains \& Strains - Back & 846-847 & 12 & 1.4 & 0.7 & 2.6 \\
\hline -Sprains \& Strains - Other & $840-845,848$ & 33 & 3.0 & 2.1 & 4.4 \\
\hline -Internal Injury - Thorax, Abdomen, Pelvis & $860-869$ & 1 & 0.1 & 0.0 & $\overline{0.6}$ \\
\hline -Open Wound - Head, Neck, Trunk & 870-879 & 6 & 1.1 & 0.4 & 2.7 \\
\hline -Superficial Injury & $910-919$ & 1 & 0.1 & 0.0 & 0.5 \\
\hline -Contusion & $920-924$ & 2 & 0.2 & 0.0 & 0.8 \\
\hline -Burns & $940-949$ & 2 & 0.1 & 0.0 & 0.5 \\
\hline -Complications \& Unspecified Injuries & 958-959 & 5 & 0.6 & 0.2 & 1.5 \\
\hline -Unspecified Effects - External Causes & $990-995$ & 2 & 0.1 & 0.0 & $\overline{0.6}$ \\
\hline -Complications of Surgical/Medical Care & 996-999 & 5 & 0.6 & 0.2 & 1.7 \\
\hline Total & & 692 & 68.8 & 63.2 & 74.8 \\
\hline
\end{tabular}

* Only those diagnostic categories with at least one occurrence appear in this table.

**Standardized to age distribution of 2000 U.S. population. 
Lawrence Livermore National Laboratory 2007

OSHA Data

Appendix L. Number of Workers with at Least One OSHA Event by Gender, Age, and Job Category*

\begin{tabular}{|c|c|c|c|c|c|c|c|c|c|c|c|}
\hline \multirow{3}{*}{ Job Category } & \multicolumn{5}{|c|}{ Women } & \multicolumn{5}{|c|}{ Men } & \multirow[b]{3}{*}{ TOTAL } \\
\hline & \multicolumn{4}{|c|}{ Age Group } & \multirow[b]{2}{*}{ TOTAL } & \multicolumn{4}{|c|}{ Age Group } & \multirow[b]{2}{*}{ TOTAL } & \\
\hline & $16-29$ & 30 - 39 & $40-49$ & $50+$ & & $16-29$ & 30 - 39 & $40-49$ & $50+$ & & \\
\hline Professional & 0 & 1 & 1 & 3 & 5 & 1 & 3 & 3 & 3 & 10 & 15 \\
\hline Administrative Support & 1 & 8 & 21 & 13 & 43 & 1 & 4 & 9 & 10 & 24 & 67 \\
\hline Technical Support & 4 & 0 & 0 & 2 & 6 & 3 & 6 & 8 & 8 & 25 & 31 \\
\hline Service & 0 & 1 & 0 & 3 & 4 & 0 & 0 & 2 & 1 & 3 & 7 \\
\hline Security and Fire & 0 & 0 & 0 & 0 & 0 & 3 & 7 & 9 & 1 & 20 & 20 \\
\hline Crafts & 0 & 0 & 0 & 1 & 1 & 0 & 1 & 7 & 6 & 14 & 15 \\
\hline TOTAL & 5 & 10 & 22 & 22 & 59 & 8 & 21 & 38 & 29 & 96 & 155 \\
\hline
\end{tabular}

*Only those job categories and gender/age combinations with at least one OSHA event appear in this table.

Appendix M. Total Number of Workdays Lost or with Restricted Activity from OSHA Events by Gender and Age

\begin{tabular}{|c|c|c|c|c|c|c|c|c|c|}
\hline \multirow{2}{*}{$\begin{array}{l}\text { Age } \\
\text { Group }\end{array}$} & \multicolumn{3}{|c|}{ Women } & \multicolumn{3}{|c|}{ Men } & \multicolumn{3}{|c|}{ TOTAL } \\
\hline & $\begin{array}{c}\text { Number } \\
\text { of } \\
\text { Events }\end{array}$ & $\begin{array}{c}\text { Days } \\
\text { Restricted }\end{array}$ & $\begin{array}{l}\text { Days } \\
\text { Lost }\end{array}$ & $\begin{array}{c}\text { Number } \\
\text { of } \\
\text { Events }\end{array}$ & $\begin{array}{c}\text { Days } \\
\text { Restricted }\end{array}$ & $\begin{array}{l}\text { Days } \\
\text { Lost }\end{array}$ & $\begin{array}{c}\text { Number } \\
\text { of } \\
\text { Events }\end{array}$ & $\begin{array}{c}\text { Days } \\
\text { Restricted }\end{array}$ & $\begin{array}{l}\text { Days } \\
\text { Lost }\end{array}$ \\
\hline $16-29$ & 5 & 71 & 0 & 8 & 282 & 17 & 13 & 353 & 17 \\
\hline $30-39$ & 10 & 114 & 54 & 21 & 240 & 123 & 31 & 354 & 177 \\
\hline $40-49$ & 22 & 0 & 80 & 41 & 920 & 119 & 63 & 920 & 199 \\
\hline $50+$ & 22 & 269 & 547 & 29 & 93 & 93 & 51 & 362 & 640 \\
\hline TOTAL & 59 & 454 & 681 & 99 & 1535 & 352 & 158 & 1989 & 1033 \\
\hline
\end{tabular}

Appendix N. Total Number of Workdays Lost or with Restricted Activity from OSHA Events by Gender and Job Category*

\begin{tabular}{|l|r|r|r|r|r|r|r|r|r|}
\hline \multirow{2}{*}{ Job Category } & \multicolumn{4}{|c|}{ Women } & \multicolumn{3}{c|}{ Men } & \multicolumn{3}{c|}{ Total } \\
\cline { 2 - 10 } & $\begin{array}{c}\text { Number } \\
\text { of } \\
\text { Events }\end{array}$ & $\begin{array}{c}\text { Days } \\
\text { Restricted }\end{array}$ & $\begin{array}{c}\text { Days } \\
\text { Lost }\end{array}$ & $\begin{array}{c}\text { Number } \\
\text { of } \\
\text { Events }\end{array}$ & $\begin{array}{c}\text { Days } \\
\text { Restricted }\end{array}$ & $\begin{array}{c}\text { Number } \\
\text { Days } \\
\text { Lost } \\
\text { Events }\end{array}$ & $\begin{array}{c}\text { Days } \\
\text { Restricted }\end{array}$ & $\begin{array}{c}\text { Days } \\
\text { Lost }\end{array}$ \\
\hline Professional & 5 & 0 & 0 & 10 & 0 & 0 & 15 & 0 & 0 \\
\hline Administrative Support & 43 & 124 & 446 & 24 & 173 & 140 & 67 & 297 & 586 \\
\hline Technical Support & 6 & 106 & 207 & 26 & 334 & 53 & 32 & 440 & 260 \\
\hline Service & 4 & 86 & 0 & 3 & 30 & 0 & 7 & 116 & 0 \\
\hline Security and Fire & 0 & 0 & 0 & 21 & 776 & 135 & 21 & 776 & 135 \\
\hline Crafts & 1 & 138 & 28 & 15 & 222 & 24 & 16 & 360 & 52 \\
\hline Total & 59 & 454 & 681 & 99 & 1535 & 352 & 158 & 1989 & 1033 \\
\hline
\end{tabular}

*Only those job categories with at least one OSHA event appear in this table. 
Lawrence Livermore National Laboratory 2007

OSHA Data

Appendix O. Number of Diagnoses in Each Diagnostic Category by Gender and Age*

\begin{tabular}{|c|c|c|c|c|c|c|}
\hline & & \multicolumn{5}{|c|}{ Women } \\
\hline & & \multicolumn{4}{|c|}{ Age Group } & \multirow[b]{2}{*}{ TOTAL } \\
\hline & & $16-29$ & 30 - 39 & $40-49$ & $50+$ & \\
\hline Diagnostic Category & $\begin{array}{l}\text { ICD-9-CM } \\
\text { code }\end{array}$ & & & & & \\
\hline $\mathbf{0}$ & $\mathbf{0}$ & 0 & 0 & 0 & 0 & 0 \\
\hline INFECTIOUS \& PARASITIC DISEASES (DIS) & 001-139 & 0 & 0 & 1 & 0 & 1 \\
\hline -Mycoses & $110-118$ & 0 & 0 & 1 & 0 & 1 \\
\hline BLOOD \& BLOOD-FORMING ORGANS & $280-289$ & 0 & 0 & 0 & 0 & 0 \\
\hline NERVOUS SYSTEM (NS) \& SENSE ORGANS & $320-389$ & 1 & 1 & 2 & 0 & 4 \\
\hline -Disorders of Peripheral NS & 350-359 & 1 & 1 & 2 & 0 & 4 \\
\hline -Dis of Ear \& Mastoid & $380-389$ & 0 & 0 & 0 & 0 & 0 \\
\hline RESPIRATORY SYSTEM & $460-519$ & 1 & 0 & 0 & 0 & 1 \\
\hline -Lung Dis from External Agents & $500-508$ & 1 & 0 & 0 & 0 & 1 \\
\hline DIGESTIVE SYSTEM & $520-579$ & 0 & 0 & 0 & 1 & 1 \\
\hline -Hernia & $550-553$ & 0 & 0 & 0 & 1 & 1 \\
\hline SKIN \& SUBCUTANEOUS TISSUE & 680-709 & 1 & 1 & 0 & 0 & 2 \\
\hline -Infections & $680-686$ & 0 & 1 & 0 & 0 & 1 \\
\hline -Other Inflammatory Conditions & $690-698$ & 1 & 0 & 0 & 0 & 1 \\
\hline -Other & 700-709 & 0 & 0 & 0 & 0 & 0 \\
\hline MUSCULOSKELETAL \& CONNECTIVE TISSUE & 710-739 & 3 & 8 & 14 & 16 & 41 \\
\hline -Arthropathies & 710-719 & 1 & 6 & 13 & 10 & 30 \\
\hline -Dorsopathies & $720-724$ & 0 & 2 & 0 & 2 & 4 \\
\hline -Rheumatism, Excluding Back & 725-729 & 2 & 0 & 1 & 4 & 7 \\
\hline SYMPTOMS, SIGNS, \& ILL-DEFINED CONDITIONS & $780-799$ & 1 & 1 & 3 & 4 & 9 \\
\hline -Symptoms & 780-789 & 1 & 1 & 2 & 4 & 8 \\
\hline -Non-Specific Abnormal Findings & $790-796$ & 0 & 0 & 1 & 0 & 1 \\
\hline INJURY \& POISONING & 800-999 & 3 & 9 & 18 & 24 & 54 \\
\hline -Fracture - Upper Limb & 810-819 & 0 & 0 & 0 & 1 & 1 \\
\hline -Fracture - Lower Limb & $820-829$ & 0 & 0 & 0 & 0 & 0 \\
\hline -Dislocation & $830-839$ & 0 & 0 & 0 & 0 & 0 \\
\hline -Sprains \& Strains - Back & 846-847 & 0 & 2 & 1 & 1 & 4 \\
\hline -Sprains \& Strains - Other & $840-845,848$ & 0 & 1 & 5 & 9 & 15 \\
\hline -Open Wound - Head, Neck, Trunk & 870-879 & 0 & 0 & 0 & 0 & 0 \\
\hline -Open Wound - Upper Limb & $880-887$ & 0 & 0 & 0 & 0 & 0 \\
\hline -Open Wound - Lower Limb & 890-897 & 0 & 0 & 0 & 0 & 0 \\
\hline -Superficial Injury & 910-919 & 0 & 1 & 0 & 1 & 2 \\
\hline -Contusion & $920-924$ & 1 & 1 & 2 & 1 & 5 \\
\hline -Foreign Body Entering Orifice & 930-939 & 0 & 0 & 0 & 0 & 0 \\
\hline -Burns & $940-949$ & 0 & 0 & 0 & 0 & 0 \\
\hline -Complications \& Unspecified Injuries & 958-959 & 2 & 4 & 8 & 11 & 25 \\
\hline
\end{tabular}

(Continued)

*Only those diagnostic categories and gender/age combinations with at least one OSHA event appear in this table. 


\section{Lawrence Livermore National Laboratory 2007}

OSHA Data

Appendix O. Number of Diagnoses in Each Diagnostic Category by Gender and Age*

\begin{tabular}{|c|c|c|c|c|c|c|c|}
\hline & & \multicolumn{5}{|c|}{ Men } & \multirow[b]{3}{*}{ TOTAL } \\
\hline & & \multicolumn{4}{|c|}{ Age Group } & \multirow[b]{2}{*}{ TOTAL } & \\
\hline & & $16-29$ & 30 - 39 & $40-49$ & $50+$ & & \\
\hline Diagnostic Category & $\begin{array}{l}\text { ICD-9-CM } \\
\text { code }\end{array}$ & & & & & & \\
\hline $\mathbf{0}$ & $\mathbf{0}$ & 0 & 0 & 0 & 1 & 1 & 1 \\
\hline INFECTIOUS \& PARASITIC DISEASES (DIS) & 001-139 & 0 & 0 & 1 & 0 & 1 & 2 \\
\hline -Mycoses & $110-118$ & 0 & 0 & 1 & 0 & 1 & 2 \\
\hline BLOOD \& BLOOD-FORMING ORGANS & $280-289$ & 0 & 0 & 0 & 1 & 1 & 1 \\
\hline NERVOUS SYSTEM (NS) \& SENSE ORGANS & $320-389$ & 0 & 0 & 4 & 0 & 4 & 8 \\
\hline -Disorders of Peripheral NS & $350-359$ & 0 & 0 & 2 & 0 & 2 & 6 \\
\hline -Dis of Ear \& Mastoid & $380-389$ & 0 & 0 & 2 & 0 & 2 & 2 \\
\hline RESPIRATORY SYSTEM & $460-519$ & 0 & 0 & 0 & 1 & 1 & 2 \\
\hline -Lung Dis from External Agents & $500-508$ & 0 & 0 & 0 & 1 & 1 & 2 \\
\hline DIGESTIVE SYSTEM & $520-579$ & 0 & 0 & 0 & 1 & 1 & 2 \\
\hline -Hernia & $550-553$ & 0 & 0 & 0 & 1 & 1 & 2 \\
\hline SKIN \& SUBCUTANEOUS TISSUE & 680-709 & 0 & 2 & 0 & 0 & 2 & 4 \\
\hline -Infections & $680-686$ & 0 & 0 & 0 & 0 & 0 & 1 \\
\hline -Other Inflammatory Conditions & $690-698$ & 0 & 1 & 0 & 0 & 1 & 2 \\
\hline -Other & 700-709 & 0 & 1 & 0 & 0 & 1 & 1 \\
\hline MUSCULOSKELETAL \& CONNECTIVE TISSUE & 710-739 & 3 & 20 & 22 & 19 & 64 & 105 \\
\hline -Arthropathies & 710-719 & 1 & 14 & 10 & 14 & 39 & 69 \\
\hline -Dorsopathies & $720-724$ & 0 & 4 & 11 & 1 & 16 & 20 \\
\hline -Rheumatism, Excluding Back & 725-729 & 2 & 2 & 1 & 4 & 9 & 16 \\
\hline SYMPTOMS, SIGNS, \& ILL-DEFINED CONDITIONS & $780-799$ & 1 & 4 & 6 & 2 & 13 & 22 \\
\hline -Symptoms & $780-789$ & 1 & 4 & 6 & 2 & 13 & 21 \\
\hline -Non-Specific Abnormal Findings & $790-796$ & 0 & 0 & 0 & 0 & 0 & 1 \\
\hline INJURY \& POISONING & 800-999 & 9 & 23 & 35 & 25 & 92 & 146 \\
\hline -Fracture - Upper Limb & 810-819 & 0 & 1 & 0 & 2 & 3 & 4 \\
\hline -Fracture - Lower Limb & $820-829$ & 0 & 0 & 0 & 1 & 1 & 1 \\
\hline -Dislocation & 830-839 & 0 & 2 & 1 & 0 & 3 & 3 \\
\hline -Sprains \& Strains - Back & 846-847 & 0 & 1 & 9 & 2 & 12 & 16 \\
\hline -Sprains \& Strains - Other & $840-845,848$ & 2 & 5 & 12 & 9 & 28 & 43 \\
\hline -Open Wound - Head, Neck, Trunk & 870-879 & 1 & 1 & 0 & 0 & 2 & 2 \\
\hline -Open Wound - Upper Limb & 880-887 & 1 & 3 & 3 & 2 & 9 & 9 \\
\hline -Open Wound - Lower Limb & 890-897 & 0 & 0 & 1 & 0 & 1 & 1 \\
\hline -Superficial Injury & $910-919$ & 1 & 0 & 0 & 0 & 1 & 3 \\
\hline -Contusion & $920-924$ & 1 & 4 & 0 & 3 & 8 & 13 \\
\hline -Foreign Body Entering Orifice & 930-939 & 0 & 0 & 1 & 1 & 2 & 2 \\
\hline -Burns & $940-949$ & 0 & 0 & 0 & 1 & 1 & 1 \\
\hline -Complications \& Unspecified Injuries & 958-959 & 3 & 5 & 7 & 4 & 19 & 44 \\
\hline
\end{tabular}

(Continued)

*Only those diagnostic categories and gender/age combinations with at least one OSHA event appear in this table. 
Lawrence Livermore National Laboratory 2007

OSHA Data

Appendix O. Number of Diagnoses in Each Diagnostic Category by Gender and Age*

\begin{tabular}{|c|c|c|c|c|c|c|}
\hline & & \multicolumn{5}{|c|}{ Women } \\
\hline & & \multicolumn{4}{|c|}{ Age Group } & \multirow[b]{2}{*}{ TOTAL } \\
\hline & & $16-29$ & $30-39$ & $40-49$ & $50+$ & \\
\hline Diagnostic Category & $\begin{array}{l}\text { ICD-9-CM } \\
\text { code }\end{array}$ & \multirow[b]{2}{*}{0} & \multirow[b]{2}{*}{0} & \multirow[b]{2}{*}{2} & \multirow[b]{2}{*}{0} & \multirow[b]{2}{*}{2} \\
\hline -Toxic Effects - Non-medicinal & 980-989 & & & & & \\
\hline -Unspecified Effects - External Causes & $990-995$ & 0 & 0 & 0 & 0 & 0 \\
\hline
\end{tabular}

*Only those diagnostic categories and gender/age combinations with at least one OSHA event appear in this table. 
Lawrence Livermore National Laboratory 2007

OSHA Data

Appendix O. Number of Diagnoses in Each Diagnostic Category by Gender and Age*

\begin{tabular}{|c|c|c|c|c|c|c|c|}
\hline & & \multicolumn{5}{|c|}{ Men } & \multirow[b]{3}{*}{ TOTAI } \\
\hline & & \multicolumn{4}{|c|}{ Age Group } & \multirow[b]{2}{*}{ TOTAL } & \\
\hline & & $16-29$ & 30 - 39 & $40-49$ & $50+$ & & \\
\hline Diagnostic Category & $\begin{array}{l}\text { ICD-9-CM } \\
\text { code }\end{array}$ & & & & & & \\
\hline -Toxic Effects - Non-medicinal & 980-989 & 0 & 1 & 0 & 0 & 1 & 3 \\
\hline -Unspecified Effects - External Causes & $990-995$ & 0 & 0 & 1 & 0 & 1 & 1 \\
\hline
\end{tabular}

\begin{tabular}{|c|c|c|c|c|c|c|c|c|c|c|c|}
\hline & \multicolumn{5}{|c|}{ Women } & \multicolumn{5}{|c|}{ Men } & \multirow[b]{3}{*}{ TOTAL } \\
\hline & \multicolumn{4}{|c|}{ Age Group } & \multirow[b]{2}{*}{ TOTAL } & \multicolumn{4}{|c|}{ Age Group } & \multirow[b]{2}{*}{ TOTAL } & \\
\hline & $16-29$ & \begin{tabular}{|l|}
$30-39$ \\
\end{tabular} & $40-49$ & $50+$ & & $16-29$ & $30-39$ & $40-49$ & $50+$ & & \\
\hline $\begin{array}{l}\text { Diagnostic } \\
\text { Category }\end{array}$ & & & & & & & & & & & \\
\hline Total & 10 & 20 & 38 & 45 & 113 & 13 & 49 & 68 & 49 & 179 & 292 \\
\hline
\end{tabular}

*Only those diagnostic categories and gender/age combinations with at least one OSHA event appear in this table. 


\section{Lawrence Livermore National Laboratory 2007}

OSHA Data

Appendix P. Number of Workdays Lost or with Restricted Activity in Each Diagnostic Category by Gender and Age*

\begin{tabular}{|c|c|c|c|c|c|c|c|c|c|}
\hline & & \multicolumn{8}{|c|}{ Women } \\
\hline & & \multicolumn{8}{|c|}{ Age Group } \\
\hline & & \multicolumn{2}{|c|}{$16-29$} & \multicolumn{2}{|c|}{$30-39$} & \multicolumn{2}{|c|}{$40-49$} & \multicolumn{2}{|l|}{$50+$} \\
\hline & & $\begin{array}{c}\text { Days } \\
\text { Restricted }\end{array}$ & $\begin{array}{l}\text { Days } \\
\text { Lost }\end{array}$ & \begin{tabular}{|c|} 
Days \\
Restricted
\end{tabular} & \begin{tabular}{l|} 
Days \\
Lost
\end{tabular} & $\begin{array}{c}\text { Days } \\
\text { Restricted }\end{array}$ & $\begin{array}{l}\text { Days } \\
\text { Lost }\end{array}$ & $\begin{array}{c}\text { Days } \\
\text { Restricted }\end{array}$ & $\begin{array}{l}\text { Days } \\
\text { Lost }\end{array}$ \\
\hline Diagnostic Category & $\begin{array}{l}\text { ICD-9-CM } \\
\text { Codes }\end{array}$ & & & & & & & & \\
\hline -Mycoses & 110-118 & 0 & 0 & 0 & 0 & 0 & 0 & 0 & 0 \\
\hline -Disorders of Peripheral NS & $350-359$ & 0 & 0 & 0 & 0 & 0 & 0 & 0 & 0 \\
\hline -Lung Dis from External Agents & $500-508$ & 0 & 0 & 0 & 0 & 0 & 0 & 0 & 0 \\
\hline -Hernia & $550-553$ & 0 & 0 & 0 & 0 & 0 & 0 & 0 & 0 \\
\hline -Infections & $680-686$ & 0 & 0 & 0 & 20 & 0 & 0 & 0 & 0 \\
\hline -Other Inflammatory Conditions & $690-698$ & 0 & 0 & 0 & 0 & 0 & 0 & 0 & 0 \\
\hline -Arthropathies & $710-719$ & 0 & 0 & 71 & 34 & 0 & 52 & 221 & 235 \\
\hline -Dorsopathies & $720-724$ & 0 & 0 & 43 & 0 & 0 & 0 & 0 & 0 \\
\hline -Rheumatism, Excluding Back & $725-729$ & 71 & 0 & 0 & 0 & 0 & 0 & 43 & 0 \\
\hline -Symptoms & $780-789$ & 0 & 0 & 0 & 0 & 0 & 52 & 48 & 132 \\
\hline -Non-Specific Abnormal Findings & $790-796$ & 0 & 0 & 0 & 0 & 0 & 0 & 0 & 0 \\
\hline -Fracture - Upper Limb & $810-819$ & 0 & 0 & 0 & 0 & 0 & 0 & 0 & 180 \\
\hline -Sprains \& Strains - Back & 846-847 & 0 & 0 & 43 & 0 & 0 & 0 & 0 & 0 \\
\hline -Sprains \& Strains - Other & $840-845,848$ & 0 & 0 & 0 & 0 & 0 & 28 & 226 & 273 \\
\hline -Superficial Injury & 910-919 & 0 & 0 & 0 & 20 & 0 & 0 & 0 & 0 \\
\hline -Contusion & $920-924$ & 0 & 0 & 0 & 0 & 0 & 0 & 0 & 0 \\
\hline -Complications \& Unspecified Injuries & 958-959 & 71 & 0 & 71 & 34 & 0 & 0 & 0 & 94 \\
\hline -Toxic Effects - Non-medicinal & $980-989$ & 0 & 0 & 0 & 0 & 0 & 0 & 0 & 0 \\
\hline
\end{tabular}

\begin{tabular}{|c|c|c|c|c|c|c|c|c|c|}
\hline & & \multicolumn{8}{|c|}{ Men } \\
\hline & & \multicolumn{8}{|c|}{ Age Group } \\
\hline & & \multicolumn{2}{|c|}{$16-29$} & \multicolumn{2}{|c|}{30 - 39} & \multicolumn{2}{|c|}{$40-49$} & \multicolumn{2}{|l|}{$50+$} \\
\hline & & $\begin{array}{c}\text { Days } \\
\text { Restricted }\end{array}$ & $\begin{array}{l}\text { Days } \\
\text { Lost }\end{array}$ & $\begin{array}{c}\text { Days } \\
\text { Restricted }\end{array}$ & $\begin{array}{l}\text { Days } \\
\text { Lost }\end{array}$ & $\begin{array}{c}\text { Days } \\
\text { Restricted }\end{array}$ & $\begin{array}{l}\text { Days } \\
\text { Lost }\end{array}$ & $\begin{array}{c}\text { Days } \\
\text { Restricted }\end{array}$ & $\begin{array}{l}\text { Days } \\
\text { Lost }\end{array}$ \\
\hline Diagnostic Category & $\begin{array}{l}\text { ICD-9-CM } \\
\text { Codes }\end{array}$ & & & & & & & & \\
\hline $\mathbf{0}$ & $\mathbf{0}$ & 0 & 0 & 0 & 0 & 0 & 0 & 0 & 31 \\
\hline -Mycoses & 110-118 & 0 & 0 & 0 & 0 & 35 & 0 & 0 & 0 \\
\hline -Disorders of Peripheral NS & 350-359 & 0 & 0 & 0 & 0 & 27 & 11 & 0 & 0 \\
\hline -Dis of Ear \& Mastoid & 380-389 & 0 & 0 & 0 & 0 & 0 & 0 & 0 & 0 \\
\hline -Lung Dis from External Agents & $500-508$ & 0 & 0 & 0 & 0 & 0 & 0 & 62 & 0 \\
\hline -Hernia & $550-553$ & 0 & 0 & 0 & 0 & 0 & 0 & 0 & 56 \\
\hline -Other Inflammatory Conditions & $690-698$ & 0 & 0 & 0 & 0 & 0 & 0 & 0 & 0 \\
\hline -Other & 700-709 & 0 & 0 & 0 & 0 & 0 & 0 & 0 & 0 \\
\hline -Arthropathies & $710-719$ & 25 & 0 & 222 & 0 & 265 & 49 & 0 & 0 \\
\hline
\end{tabular}

(Continued)

*OSHA events with >1 ICD-9-CM code in the same diagnostic category were counted only once. Only those diagnostic categories and gender/age combinations with at least one occurrence appear in this table. 


\section{Lawrence Livermore National Laboratory 2007}

OSHA Data

Appendix P. Number of Workdays Lost or with Restricted Activity in Each Diagnostic Category by Gender and Age*

\begin{tabular}{|c|c|c|c|c|c|c|c|c|c|}
\hline & & \multicolumn{8}{|c|}{ Men } \\
\hline & & \multicolumn{8}{|c|}{ Age Group } \\
\hline & & \multicolumn{2}{|c|}{$16-29$} & \multicolumn{2}{|c|}{$30-39$} & \multicolumn{2}{|c|}{$40-49$} & \multicolumn{2}{|l|}{$50+$} \\
\hline & & $\begin{array}{c}\text { Days } \\
\text { Restricted }\end{array}$ & $\begin{array}{c}\text { Days } \\
\text { Lost }\end{array}$ & $\begin{array}{c}\text { Days } \\
\text { Restricted }\end{array}$ & \begin{tabular}{l|} 
Days \\
Lost
\end{tabular} & $\begin{array}{c}\text { Days } \\
\text { Restricted }\end{array}$ & $\begin{array}{l}\text { Days } \\
\text { Lost }\end{array}$ & \begin{tabular}{c|} 
Days \\
Restricted
\end{tabular} & $\begin{array}{l}\text { Days } \\
\text { Lost }\end{array}$ \\
\hline Diagnostic Category & $\begin{array}{l}\text { ICD-9-CM } \\
\text { Codes }\end{array}$ & & & & & & & & \\
\hline -Dorsopathies & $720-724$ & 0 & 0 & 16 & 0 & 262 & 38 & 0 & 0 \\
\hline -Rheumatism, Excluding Back & 725-729 & 64 & 0 & 0 & 0 & 110 & 0 & 0 & 0 \\
\hline -Symptoms & 780-789 & 9 & 14 & 2 & 0 & 35 & 13 & 0 & 31 \\
\hline -Fracture - Upper Limb & 810-819 & 0 & 0 & 0 & 0 & 0 & 0 & 0 & 0 \\
\hline -Fracture - Lower Limb & 820-829 & 0 & 0 & 0 & 0 & 0 & 0 & 0 & 0 \\
\hline -Dislocation & $830-839$ & 0 & 0 & 0 & 123 & 0 & 19 & 0 & 0 \\
\hline -Sprains \& Strains - Back & $846-847$ & 0 & 0 & 0 & 0 & 227 & 38 & 30 & $\overline{6}$ \\
\hline -Sprains \& Strains - Other & $840-845,848$ & 34 & 14 & 201 & 0 & 340 & 49 & 0 & 0 \\
\hline -Open Wound - Head, Neck, Trunk & 870-879 & 0 & 0 & 2 & 0 & 0 & 0 & 0 & 0 \\
\hline -Open Wound - Upper Limb & $880-887$ & 4 & 0 & 0 & 0 & 19 & 0 & 0 & 0 \\
\hline -Open Wound - Lower Limb & $890-897$ & 0 & 0 & 0 & 0 & 0 & 0 & 0 & 0 \\
\hline -Superficial Injury & $910-919$ & 0 & 0 & 0 & 0 & 0 & 0 & 0 & $\overline{0}$ \\
\hline -Contusion & $920-924$ & 0 & 3 & 23 & 0 & 0 & 0 & 0 & 0 \\
\hline -Foreign Body Entering Orifice & $930-939$ & 0 & 0 & 0 & 0 & 0 & 0 & 0 & 0 \\
\hline -Burns & $940-949$ & 0 & 0 & 0 & 0 & 0 & 0 & 1 & 0 \\
\hline -Complications \& Unspecified Injuries & 958-959 & 244 & 0 & 16 & 123 & 321 & 22 & 0 & 0 \\
\hline -Toxic Effects - Non-medicinal & 980-989 & 0 & 0 & 0 & 0 & 0 & 0 & 0 & 0 \\
\hline -Unspecified Effects - External Causes & $990-995$ & 0 & 0 & 0 & 0 & 109 & 0 & 0 & 0 \\
\hline
\end{tabular}

*OSHA events with >1 ICD-9-CM code in the same diagnostic category were counted only once. Only those diagnostic categories and gender/age combinations with at least one occurrence appear in this table. 
Appendix Q. Number of Occurrences in Each Accident Category by Gender and Age*

\begin{tabular}{|c|c|c|c|c|c|c|c|c|c|c|c|c|}
\hline & & \multicolumn{5}{|c|}{ Women } & \multicolumn{5}{|c|}{ Men } & \multirow[b]{3}{*}{ TOTAL } \\
\hline & & \multicolumn{4}{|c|}{ Age Group } & \multirow[b]{2}{*}{ TOTAL } & \multicolumn{4}{|c|}{ Age Group } & \multirow[b]{2}{*}{ TOTAL } & \\
\hline & & $16-29$ & $30-39$ & $40-49$ & $\begin{array}{l}50 \\
+\end{array}$ & & $16-29$ & 30 - 39 & $40-49$ & $\begin{array}{l}50 \\
+\end{array}$ & & \\
\hline Type of Accident & E Codes & & & & & & & & & & & \\
\hline Motor Vehicle Traffic & E810-E819 & 0 & 0 & 1 & 0 & 1 & 0 & 0 & 0 & 0 & 0 & 1 \\
\hline Other Road Vehicle & E826-E829 & 0 & 0 & 0 & 0 & 0 & 0 & 0 & 0 & 2 & 2 & 2 \\
\hline $\begin{array}{l}\text { Accidental } \\
\text { Poisoning - Non-medicinal }\end{array}$ & E860-E869 & 1 & 0 & 0 & 0 & 1 & 0 & 0 & 0 & 0 & 0 & 1 \\
\hline Falls & E880-E888 & 0 & 2 & 5 & 4 & 11 & 1 & 1 & 4 & 6 & 12 & 23 \\
\hline Natural/Environmental Factors & E900-E909 & 0 & 1 & 2 & 0 & 3 & 0 & 1 & 0 & 0 & 1 & 4 \\
\hline $\begin{array}{l}\text { Submersion/Suffocation/Foreign } \\
\text { Bodies }\end{array}$ & E910-E915 & 0 & 0 & 0 & 0 & 0 & 0 & 0 & 1 & 1 & 2 & 2 \\
\hline Other Accidents & E916-E928 & 4 & 7 & 12 & 18 & 41 & 7 & 18 & 30 & 16 & 71 & 112 \\
\hline
\end{tabular}

*Only those accident types and gender/age combinations with at least one occurrence appear in this table.

Appendix R. Number of Workdays Lost or with Restricted Activity in Each Accident Category by Gender and Age*

\begin{tabular}{|c|c|c|c|c|c|c|c|c|c|}
\hline & & \multicolumn{8}{|c|}{ Women } \\
\hline & & \multicolumn{8}{|c|}{ Age Group } \\
\hline & & \multicolumn{2}{|c|}{$16-29$} & \multicolumn{2}{|c|}{30 - 39} & \multicolumn{2}{|c|}{$40-49$} & \multicolumn{2}{|l|}{$\mathbf{5 0 +}$} \\
\hline & & $\begin{array}{c}\text { Days } \\
\text { Restricted }\end{array}$ & \begin{tabular}{|l|} 
Days \\
Lost
\end{tabular} & \begin{tabular}{|c|} 
Days \\
Restricted
\end{tabular} & $\begin{array}{l}\text { Days } \\
\text { Lost }\end{array}$ & \begin{tabular}{|c|} 
Days \\
Restricted
\end{tabular} & $\begin{array}{l}\text { Days } \\
\text { Lost }\end{array}$ & $\begin{array}{c}\text { Days } \\
\text { Restricted }\end{array}$ & $\begin{array}{l}\text { Days } \\
\text { Lost }\end{array}$ \\
\hline Type of Accident & E Codes & & & & & & & & \\
\hline Motor Vehicle Traffic & E810-E819 & 0 & 0 & 0 & 0 & 0 & 0 & 0 & 0 \\
\hline Accidental Poisoning - Non-medicinal & E860-E869 & 0 & 0 & 0 & 0 & 0 & 0 & 0 & 0 \\
\hline Falls & E880-E888 & 0 & 0 & 0 & 0 & 0 & 28 & 0 & 180 \\
\hline Natural/Environmental Factors & E900-E909 & 0 & 0 & 0 & 20 & 0 & 0 & 0 & 0 \\
\hline Other Accidents & E916-E928 & 71 & 0 & 114 & 34 & 0 & 52 & 269 & 367 \\
\hline
\end{tabular}

\begin{tabular}{|c|c|c|c|c|c|c|c|c|c|}
\hline & & \multicolumn{8}{|c|}{ Men } \\
\hline & & \multicolumn{8}{|c|}{ Age Group } \\
\hline & & \multicolumn{2}{|c|}{$16-29$} & \multicolumn{2}{|c|}{30 - 39} & \multicolumn{2}{|c|}{$40-49$} & \multicolumn{2}{|l|}{$50+$} \\
\hline & & $\begin{array}{c}\text { Days } \\
\text { Restricted }\end{array}$ & $\begin{array}{l}\text { Days } \\
\text { Lost }\end{array}$ & \begin{tabular}{|c|} 
Days \\
Restricted
\end{tabular} & \begin{tabular}{|l|} 
Days \\
Lost
\end{tabular} & \begin{tabular}{|c|} 
Days \\
Restricted
\end{tabular} & $\begin{array}{l}\text { Days } \\
\text { Lost }\end{array}$ & \begin{tabular}{|c|} 
Days \\
Restricted
\end{tabular} & $\begin{array}{l}\text { Days } \\
\text { Lost }\end{array}$ \\
\hline Type of Accident & E Codes & \multirow[b]{2}{*}{0} & \multirow[b]{2}{*}{0} & \multirow[b]{2}{*}{0} & \multirow[b]{2}{*}{0} & \multirow[b]{2}{*}{0} & \multirow[b]{2}{*}{0} & \multirow[b]{2}{*}{0} & \multirow[b]{2}{*}{0} \\
\hline Other Road Vehicle & E826-E829 & & & & & & & & \\
\hline Falls & E880-E888 & 0 & 0 & 0 & 123 & 105 & 43 & 0 & 0 \\
\hline Natural/Environmental Factors & E900-E909 & 0 & 0 & 0 & 0 & 0 & 0 & 0 & 0 \\
\hline Submersion/Suffocation/Foreign Bodies & E910-E915 & 0 & 0 & 0 & 0 & 0 & 0 & 0 & 0 \\
\hline Other Accidents & E916-E928 & 282 & 17 & 240 & 0 & 513 & 43 & 31 & $\overline{6}$ \\
\hline
\end{tabular}

*OSHA events with $>1 \mathrm{E}$ code in the same accident type were counted only once. Only those accident types and gender/age combinations with at least one occurrence appear in this table. 


\section{Lawrence Livermore National Laboratory 2007}

OSHA Data

Appendix S. Number of Diagnoses in Each Diagnostic Category by Gender and Job Category*

\begin{tabular}{|c|c|c|c|c|c|c|c|}
\hline & & \multicolumn{6}{|c|}{ Women } \\
\hline & & \multicolumn{5}{|c|}{ Job Category } & \multirow[b]{2}{*}{ TOTAI } \\
\hline & & Professional & $\begin{array}{c}\text { Administrative } \\
\text { Support }\end{array}$ & $\begin{array}{l}\text { Technical } \\
\text { Support }\end{array}$ & Service & Crafts & \\
\hline Diagnostic Category & $\begin{array}{l}\text { ICD-9-CM } \\
\text { Code }\end{array}$ & & & & & & \\
\hline INFECTIOUS \& PARASITIC DISEASES (DIS) & 001-139 & 0 & 1 & 0 & 0 & 0 & 1 \\
\hline -Mycoses & 110-118 & 0 & 1 & 0 & 0 & 0 & 1 \\
\hline NERVOUS SYSTEM (NS) \& SENSE ORGANS & 320-389 & 0 & 4 & 0 & 0 & 0 & 4 \\
\hline -Disorders of Peripheral NS & 350-359 & 0 & 4 & 0 & 0 & 0 & 4 \\
\hline RESPIRATORY SYSTEM & $460-519$ & 0 & 0 & 1 & 0 & 0 & 1 \\
\hline -Lung Dis from External Agents & $500-508$ & 0 & 0 & 1 & 0 & 0 & 1 \\
\hline DIGESTIVE SYSTEM & $520-579$ & 0 & 1 & 0 & 0 & 0 & 1 \\
\hline -Hernia & $550-553$ & 0 & 1 & 0 & 0 & 0 & 1 \\
\hline SKIN \& SUBCUTANEOUS TISSUE & $680-709$ & 0 & 2 & 0 & 0 & 0 & 2 \\
\hline -Infections & $680-686$ & 0 & 1 & 0 & 0 & 0 & 1 \\
\hline -Other Inflammatory Conditions & $690-698$ & 0 & 1 & 0 & 0 & 0 & 1 \\
\hline MUSCULOSKELETAL \& CONNECTIVE TISSUE & 710-739 & 4 & 25 & 5 & 6 & 1 & 41 \\
\hline -Arthropathies & $710-719$ & 2 & 22 & 3 & 2 & 1 & 30 \\
\hline -Dorsopathies & $720-724$ & 1 & 1 & 0 & 2 & 0 & 4 \\
\hline -Rheumatism, Excluding Back & 725-729 & 1 & 2 & 2 & 2 & 0 & 7 \\
\hline $\begin{array}{l}\text { SYMPTOMS, SIGNS, \& ILL-DEFINED } \\
\text { CONDITIONS }\end{array}$ & $780-799$ & 0 & 8 & 0 & 1 & 0 & 9 \\
\hline -Symptoms & 780-789 & 0 & 7 & 0 & 1 & 0 & 8 \\
\hline -Non-Specific Abnormal Findings & $790-796$ & 0 & 1 & 0 & 0 & 0 & 1 \\
\hline INJURY \& POISONING & 800-999 & 6 & 39 & 5 & 3 & 1 & 54 \\
\hline -Fracture - Upper Limb & 810-819 & 0 & 1 & 0 & 0 & 0 & 1 \\
\hline -Sprains \& Strains - Back & 846-847 & 0 & 3 & 0 & 1 & 0 & 4 \\
\hline -Sprains \& Strains - Other & $\begin{array}{l}840-845 \\
848\end{array}$ & 1 & 11 & 1 & 1 & 1 & 15 \\
\hline -Superficial Injury & $910-919$ & 0 & 1 & 0 & 1 & 0 & 2 \\
\hline -Contusion & $920-924$ & 1 & 3 & 1 & 0 & 0 & 5 \\
\hline -Complications \& Unspecified Injuries & 958-959 & 4 & 18 & 3 & 0 & 0 & 25 \\
\hline -Toxic Effects - Non-medicinal & 980-989 & 0 & 2 & 0 & 0 & 0 & 2 \\
\hline
\end{tabular}

\begin{tabular}{|c|c|c|c|c|c|c|}
\hline & \multicolumn{6}{|c|}{ Women } \\
\hline & \multicolumn{5}{|c|}{ Job Category } & \multirow[b]{2}{*}{ TOTAL } \\
\hline & Professional & \begin{tabular}{|c|} 
Administrative \\
Support
\end{tabular} & $\begin{array}{c}\text { Technical } \\
\text { Support }\end{array}$ & Service & Crafts & \\
\hline $\begin{array}{l}\text { Diagnostic } \\
\text { Category }\end{array}$ & & & & & & \\
\hline Total & 10 & 80 & 11 & 10 & 2 & 113 \\
\hline
\end{tabular}

*Only those diagnostic categories and gender/job category combinations with at least one occurrence appear in this table. 


\section{Lawrence Livermore National Laboratory 2007}

OSHA Data

Appendix S. Number of Diagnoses in Each Diagnostic Category by Gender and Job Category*

\begin{tabular}{|c|c|c|c|c|c|c|c|c|}
\hline & & \multicolumn{7}{|c|}{ Men } \\
\hline & & \multicolumn{6}{|c|}{ Job Category } & \multirow[b]{2}{*}{ TOTAI } \\
\hline & & Professional & $\begin{array}{l}\text { Administrative } \\
\text { Support }\end{array}$ & $\begin{array}{l}\text { Technical } \\
\text { Support }\end{array}$ & Service & $\begin{array}{c}\text { Security } \\
\text { and } \\
\text { Fire }\end{array}$ & Crafts & \\
\hline Diagnostic Category & $\begin{array}{l}\text { ICD-9-CM } \\
\text { Code }\end{array}$ & & & & & & & \\
\hline $\mathbf{0}$ & $\mathbf{0}$ & 0 & 0 & 1 & 0 & 0 & 0 & 1 \\
\hline $\begin{array}{l}\text { INFECTIOUS \& PARASITIC DISEASES } \\
\text { (DIS) }\end{array}$ & 001-139 & 0 & 1 & 0 & 0 & 0 & 0 & 1 \\
\hline -Mycoses & 110-118 & 0 & 1 & 0 & 0 & 0 & 0 & 1 \\
\hline BLOOD \& BLOOD-FORMING ORGANS & $280-289$ & 0 & 0 & 1 & 0 & 0 & 0 & 1 \\
\hline $\begin{array}{l}\text { NERVOUS SYSTEM (NS) \& SENSE } \\
\text { ORGANS }\end{array}$ & 320-389 & 0 & 1 & 1 & 2 & 0 & 0 & 4 \\
\hline -Disorders of Peripheral NS & $350-359$ & 0 & 1 & 1 & 0 & 0 & 0 & 2 \\
\hline -Dis of Ear \& Mastoid & 380-389 & 0 & 0 & 0 & 2 & 0 & 0 & 2 \\
\hline RESPIRATORY SYSTEM & $460-519$ & 0 & 0 & 1 & 0 & 0 & 0 & 1 \\
\hline -Lung Dis from External Agents & $500-508$ & 0 & 0 & 1 & 0 & 0 & 0 & 1 \\
\hline DIGESTIVE SYSTEM & $520-579$ & 0 & 0 & 0 & 0 & 1 & 0 & 1 \\
\hline -Hernia & $550-553$ & 0 & 0 & 0 & 0 & 1 & 0 & 1 \\
\hline SKIN \& SUBCUTANEOUS TISSUE & 680-709 & 0 & 0 & 0 & 0 & 2 & 0 & 2 \\
\hline -Other Inflammatory Conditions & $690-698$ & 0 & 0 & 0 & 0 & 1 & 0 & 1 \\
\hline -Other & 700-709 & 0 & 0 & 0 & 0 & 1 & 0 & 1 \\
\hline $\begin{array}{l}\text { MUSCULOSKELETAL \& CONNECTIVE } \\
\text { TISSUE }\end{array}$ & 710-739 & 12 & 7 & 16 & 1 & 18 & 10 & 64 \\
\hline -Arthropathies & $710-719$ & 7 & 4 & 11 & 0 & 10 & 7 & 39 \\
\hline -Dorsopathies & $720-724$ & 2 & 3 & 2 & 1 & 7 & 1 & 16 \\
\hline -Rheumatism, Excluding Back & 725-729 & 3 & 0 & 3 & 0 & 1 & 2 & 9 \\
\hline $\begin{array}{l}\text { SYMPTOMS, SIGNS, \& ILL-DEFINED } \\
\text { CONDITIONS }\end{array}$ & $780-799$ & 1 & 2 & 2 & 0 & 6 & 2 & 13 \\
\hline -Symptoms & $780-789$ & 1 & 2 & 2 & 0 & 6 & 2 & 13 \\
\hline INJURY \& POISONING & 800-999 & 11 & 24 & 25 & 2 & 17 & 13 & 92 \\
\hline -Fracture - Upper Limb & 810-819 & 0 & 2 & 1 & 0 & 0 & 0 & 3 \\
\hline -Fracture - Lower Limb & 820-829 & 0 & 0 & 1 & 0 & 0 & 0 & 1 \\
\hline -Dislocation & 830-839 & 0 & 1 & 2 & 0 & 0 & 0 & 3 \\
\hline -Sprains \& Strains - Back & 846-847 & 1 & 4 & 1 & 1 & 3 & 2 & 12 \\
\hline -Sprains \& Strains - Other & $\begin{array}{l}840-845 \\
848\end{array}$ & 2 & 7 & 5 & 1 & 9 & 4 & 28 \\
\hline -Open Wound - Head, Neck, Trunk & 870-879 & 0 & 1 & 1 & 0 & 0 & 0 & 2 \\
\hline -Open Wound - Upper Limb & 880-887 & 0 & 3 & 3 & 0 & 0 & 3 & 9 \\
\hline -Open Wound - Lower Limb & 890-897 & 0 & 1 & 0 & 0 & 0 & 0 & 1 \\
\hline -Superficial Injury & 910-919 & 0 & 1 & 0 & 0 & 0 & 0 & 1 \\
\hline -Contusion & $920-924$ & 1 & 1 & 4 & 0 & 2 & 0 & 8 \\
\hline
\end{tabular}

(Continued)

*Only those diagnostic categories and gender/job category combinations with at least one occurrence appear in this table. 


\section{Lawrence Livermore National Laboratory 2007}

OSHA Data

Appendix S. Number of Diagnoses in Each Diagnostic Category by Gender and Job Category*

\begin{tabular}{|c|c|c|c|c|c|c|c|c|}
\hline & & \multicolumn{7}{|c|}{ Men } \\
\hline & & \multicolumn{6}{|c|}{ Job Category } & \multirow[b]{2}{*}{ TOTAL } \\
\hline & & Professional & $\begin{array}{c}\text { Administrative } \\
\text { Support }\end{array}$ & $\begin{array}{c}\text { Technical } \\
\text { Support }\end{array}$ & Service & $\begin{array}{c}\text { Security } \\
\text { and } \\
\text { Fire }\end{array}$ & Crafts & \\
\hline Diagnostic Category & $\begin{array}{l}\text { ICD-9-CM } \\
\text { Code }\end{array}$ & \multirow[b]{2}{*}{0} & \multirow[b]{2}{*}{0} & \multirow[b]{2}{*}{1} & \multirow[b]{2}{*}{0} & \multirow[b]{2}{*}{0} & \multirow[b]{2}{*}{1} & \multirow[b]{2}{*}{2} \\
\hline -Foreign Body Entering Orifice & 930-939 & & & & & & & \\
\hline -Burns & $940-949$ & 0 & 0 & 0 & 0 & 0 & 1 & 1 \\
\hline -Complications \& Unspecified Injuries & 958-959 & 7 & 3 & 4 & 0 & 3 & 2 & 19 \\
\hline -Toxic Effects - Non-medicinal & 980-989 & 0 & 0 & 1 & 0 & 0 & 0 & 1 \\
\hline -Unspecified Effects - External Causes & $990-995$ & 0 & 0 & 1 & 0 & 0 & 0 & 1 \\
\hline
\end{tabular}

\begin{tabular}{|c|c|c|c|c|c|c|c|}
\hline & \multicolumn{7}{|c|}{ Men } \\
\hline & \multicolumn{6}{|c|}{ Job Category } & \multirow[b]{2}{*}{ TOTAL } \\
\hline & Professional & $\begin{array}{c}\text { Administrative } \\
\text { Support }\end{array}$ & $\begin{array}{c}\text { Technical } \\
\text { Support }\end{array}$ & Service & $\begin{array}{c}\text { Security } \\
\text { and } \\
\text { Fire }\end{array}$ & Crafts & \\
\hline $\begin{array}{l}\text { Diagnostic } \\
\text { Category }\end{array}$ & & & & & & & \\
\hline Total & 24 & 35 & 46 & 5 & 44 & 25 & 179 \\
\hline
\end{tabular}

*Only those diagnostic categories and gender/job category combinations with at least one occurrence appear in this table. 


\section{Lawrence Livermore National Laboratory 2007}

OSHA Data

Appendix T. Number of Workdays Lost or with Restricted Activity in Each Diagnostic Category by Gender and Job Category*

\begin{tabular}{|c|c|c|c|c|c|c|c|}
\hline & & \multicolumn{6}{|c|}{ Women } \\
\hline & & \multicolumn{6}{|c|}{ Job Category } \\
\hline & & \multicolumn{2}{|c|}{ Professional } & \multicolumn{2}{|c|}{$\begin{array}{c}\text { Administrative } \\
\text { Support }\end{array}$} & \multicolumn{2}{|c|}{$\begin{array}{l}\text { Technical } \\
\text { Support }\end{array}$} \\
\hline & & $\begin{array}{c}\text { Days } \\
\text { Restricted }\end{array}$ & $\begin{array}{l}\text { Days } \\
\text { Lost }\end{array}$ & \begin{tabular}{c|} 
Days \\
Restricted
\end{tabular} & $\begin{array}{l}\text { Days } \\
\text { Lost }\end{array}$ & \begin{tabular}{c|} 
Days \\
Restricted
\end{tabular} & $\begin{array}{l}\text { Days } \\
\text { Lost }\end{array}$ \\
\hline Diagnostic Category & $\begin{array}{l}\text { ICD-9-CM } \\
\text { Codes }\end{array}$ & & & & & & \\
\hline -Mycoses & 110-118 & 0 & 0 & 0 & 0 & 0 & 0 \\
\hline -Disorders of Peripheral NS & $350-359$ & 0 & 0 & 0 & 0 & 0 & 0 \\
\hline -Lung Dis from External Agents & $500-508$ & 0 & 0 & 0 & 0 & 0 & 0 \\
\hline -Hernia & $\mathbf{5 5 0 - 5 5 3}$ & 0 & 0 & 0 & 0 & 0 & 0 \\
\hline -Infections & $680-686$ & 0 & 0 & 0 & 20 & 0 & 0 \\
\hline -Other Inflammatory Conditions & 690-698 & 0 & 0 & 0 & 0 & 0 & 0 \\
\hline -Arthropathies & $710-719$ & 0 & 0 & 76 & 86 & 35 & 207 \\
\hline -Dorsopathies & $720-724$ & 0 & 0 & 0 & 0 & 0 & 0 \\
\hline -Rheumatism, Excluding Back & $725-729$ & 0 & 0 & 0 & 0 & 71 & 0 \\
\hline -Symptoms & $780-789$ & 0 & 0 & 48 & 184 & 0 & 0 \\
\hline -Non-Specific Abnormal Findings & $790-796$ & 0 & 0 & 0 & 0 & 0 & 0 \\
\hline -Fracture - Upper Limb & $810-819$ & 0 & 0 & 0 & 180 & 0 & 0 \\
\hline -Sprains \& Strains - Back & 846-847 & 0 & 0 & 0 & 0 & 0 & 0 \\
\hline -Sprains \& Strains - Other & $840-845,848$ & 0 & 0 & 53 & 160 & 35 & 113 \\
\hline -Superficial Injury & 910-919 & 0 & 0 & 0 & 20 & 0 & 0 \\
\hline -Contusion & $920-924$ & 0 & 0 & 0 & 0 & 0 & 0 \\
\hline -Complications \& Unspecified Injuries & 958-959 & 0 & 0 & 71 & 34 & 71 & 94 \\
\hline -Toxic Effects - Non-medicinal & $980-989$ & 0 & 0 & 0 & 0 & 0 & 0 \\
\hline
\end{tabular}

*OSHA events with >1 ICD-9-CM code in the same diagnostic category were counted only once. Only those diagnostic categories and gender/job category combinations with at least one occurrence appear in this table. 


\section{Lawrence Livermore National Laboratory 2007}

OSHA Data

Appendix T. Number of Workdays Lost or with Restricted Activity in Each Diagnostic Category by Gender and Job Category*

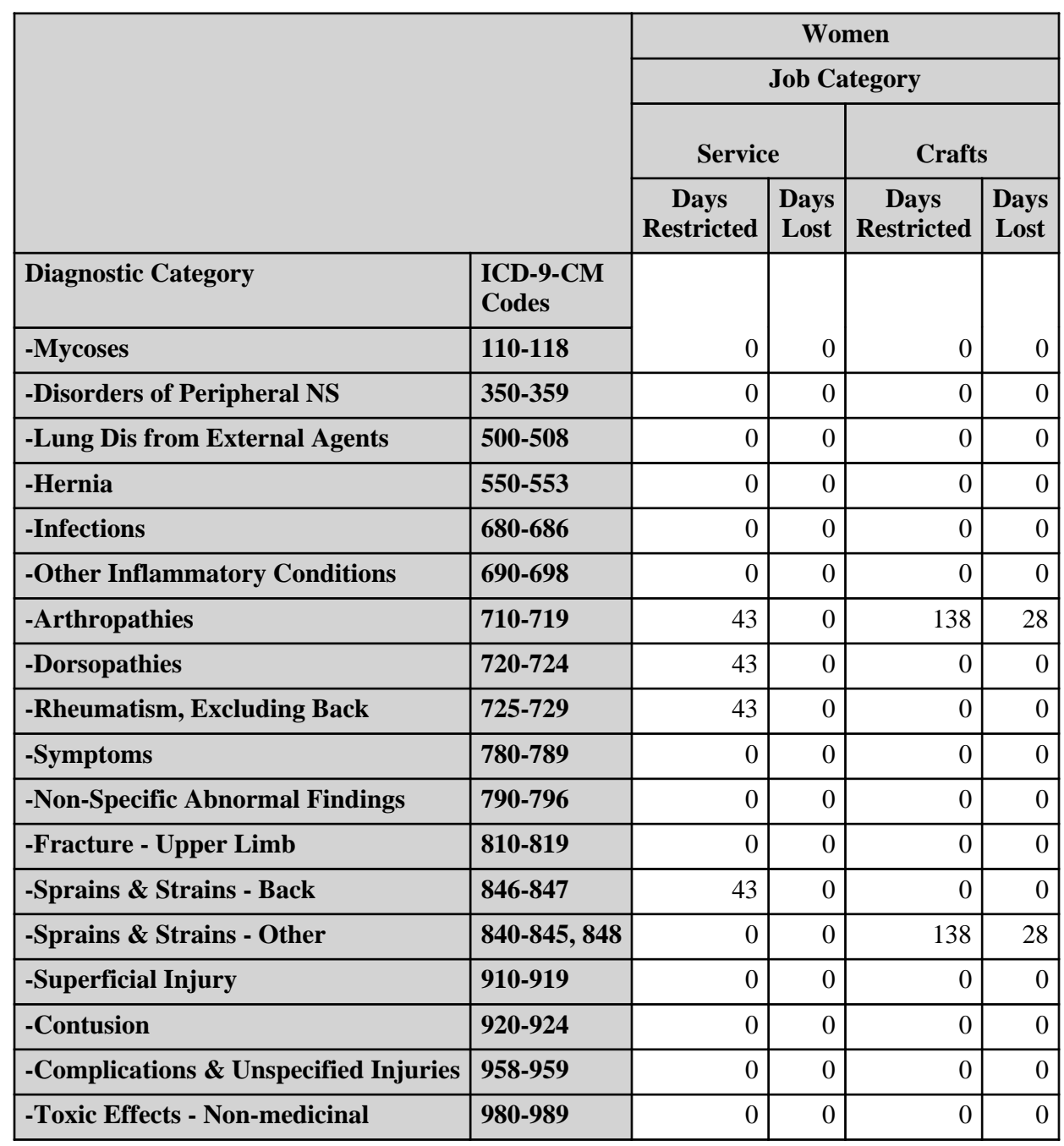

*OSHA events with >1 ICD-9-CM code in the same diagnostic category were counted only once. Only those diagnostic categories and gender/job category combinations with at least one occurrence appear in this table. 


\section{Lawrence Livermore National Laboratory 2007}

OSHA Data

Appendix T. Number of Workdays Lost or with Restricted Activity in Each Diagnostic Category by Gender and Job Category*

\begin{tabular}{|c|c|c|c|c|c|c|c|}
\hline & \multicolumn{6}{|c|}{ Men } \\
\hline & & \multicolumn{6}{|c|}{ Job Category } \\
\hline & & \multicolumn{2}{|c|}{ Professional } & \multicolumn{2}{|c|}{$\begin{array}{c}\text { Administrative } \\
\text { Support }\end{array}$} & \multicolumn{2}{|c|}{$\begin{array}{l}\text { Technical } \\
\text { Support }\end{array}$} \\
\hline & & $\begin{array}{c}\text { Days } \\
\text { Restricted }\end{array}$ & $\begin{array}{l}\text { Days } \\
\text { Lost }\end{array}$ & $\begin{array}{c}\text { Days } \\
\text { Restricted }\end{array}$ & $\begin{array}{l}\text { Days } \\
\text { Lost }\end{array}$ & \begin{tabular}{|c|} 
Days \\
Restricted
\end{tabular} & $\begin{array}{l}\text { Days } \\
\text { Lost }\end{array}$ \\
\hline Diagnostic Category & $\begin{array}{l}\text { ICD-9-CM } \\
\text { Codes }\end{array}$ & & & & & & \\
\hline $\mathbf{0}$ & $\mathbf{0}$ & 0 & 0 & 0 & 0 & 0 & 31 \\
\hline -Mycoses & 110-118 & 0 & 0 & 35 & 0 & 0 & 0 \\
\hline -Disorders of Peripheral NS & $350-359$ & 0 & 0 & 0 & 11 & 27 & 0 \\
\hline -Dis of Ear \& Mastoid & $380-389$ & 0 & 0 & 0 & 0 & 0 & $\overline{0}$ \\
\hline -Lung Dis from External Agents & \begin{tabular}{|l|}
$500-508$ \\
\end{tabular} & 0 & 0 & 0 & 0 & 62 & 0 \\
\hline -Hernia & $550-553$ & 0 & 0 & 0 & 0 & 0 & $\overline{0}$ \\
\hline -Other Inflammatory Conditions & 690-698 & 0 & 0 & 0 & 0 & 0 & 0 \\
\hline -Other & 700-709 & 0 & 0 & 0 & 0 & 0 & 0 \\
\hline -Arthropathies & 710-719 & 0 & 0 & 138 & 0 & 66 & 0 \\
\hline -Dorsopathies & $720-724$ & 0 & 0 & 35 & 0 & 0 & 0 \\
\hline -Rheumatism, Excluding Back & 725-729 & 0 & 0 & 0 & 0 & 64 & 0 \\
\hline -Symptoms & 780-789 & 0 & 0 & 35 & 11 & 2 & 31 \\
\hline -Fracture - Upper Limb & 810-819 & 0 & 0 & 0 & 0 & 0 & 0 \\
\hline -Fracture - Lower Limb & $820-829$ & 0 & 0 & 0 & 0 & 0 & 0 \\
\hline -Dislocation & $830-839$ & 0 & 0 & 0 & 123 & 0 & 19 \\
\hline -Sprains \& Strains - Back & $846-847$ & 0 & 0 & 0 & 6 & 0 & 0 \\
\hline -Sprains \& Strains - Other & $840-845,848$ & 0 & 0 & 138 & 0 & 0 & 0 \\
\hline -Open Wound - Head, Neck, Trunk & 870-879 & 0 & 0 & 0 & 0 & 2 & 0 \\
\hline -Open Wound - Upper Limb & 880-887 & 0 & 0 & 0 & 0 & 4 & 0 \\
\hline -Open Wound - Lower Limb & $890-897$ & 0 & 0 & 0 & 0 & 0 & 0 \\
\hline -Superficial Injury & $910-919$ & 0 & 0 & 0 & 0 & 0 & 0 \\
\hline -Contusion & $920-924$ & 0 & 0 & 0 & 0 & 15 & 3 \\
\hline -Foreign Body Entering Orifice & 930-939 & 0 & 0 & 0 & 0 & 0 & 0 \\
\hline -Burns & $940-949$ & 0 & 0 & 0 & 0 & 0 & 0 \\
\hline -Complications \& Unspecified Injuries & 958-959 & 0 & 0 & 0 & 123 & 117 & 0 \\
\hline -Toxic Effects - Non-medicinal & 980-989 & 0 & 0 & 0 & 0 & 0 & 0 \\
\hline -Unspecified Effects - External Causes & $990-995$ & 0 & 0 & 0 & 0 & 109 & 0 \\
\hline
\end{tabular}

*OSHA events with >1 ICD-9-CM code in the same diagnostic category were counted only once. Only those diagnostic categories and gender/job category combinations with at least one occurrence appear in this table. 


\section{Lawrence Livermore National Laboratory 2007}

OSHA Data

Appendix T. Number of Workdays Lost or with Restricted Activity in Each Diagnostic Category by Gender and Job Category*

\begin{tabular}{|c|c|c|c|c|c|c|c|}
\hline & \multicolumn{6}{|c|}{ Men } \\
\hline & & \multicolumn{6}{|c|}{ Job Category } \\
\hline & & \multicolumn{2}{|c|}{ Service } & \multicolumn{2}{|c|}{$\begin{array}{c}\text { Security and } \\
\text { Fire }\end{array}$} & \multicolumn{2}{|c|}{ Crafts } \\
\hline & & $\begin{array}{c}\text { Days } \\
\text { Restricted }\end{array}$ & \begin{tabular}{|l|} 
Days \\
Lost
\end{tabular} & \begin{tabular}{|c|} 
Days \\
Restricted
\end{tabular} & \begin{tabular}{l|} 
Days \\
Lost
\end{tabular} & $\begin{array}{c}\text { Days } \\
\text { Restricted }\end{array}$ & $\begin{array}{l}\text { Days } \\
\text { Lost }\end{array}$ \\
\hline Diagnostic Category & $\begin{array}{l}\text { ICD-9-CM } \\
\text { Codes }\end{array}$ & & & & & & \\
\hline $\mathbf{0}$ & $\mathbf{0}$ & 0 & 0 & 0 & 0 & 0 & 0 \\
\hline -Mycoses & $110-118$ & 0 & 0 & 0 & 0 & 0 & 0 \\
\hline -Disorders of Peripheral NS & $350-359$ & 0 & 0 & 0 & 0 & 0 & 0 \\
\hline -Dis of Ear \& Mastoid & $380-389$ & 0 & 0 & 0 & 0 & 0 & 0 \\
\hline -Lung Dis from External Agents & $500-508$ & 0 & 0 & 0 & 0 & 0 & 0 \\
\hline -Hernia & $550-553$ & 0 & 0 & 0 & 56 & 0 & 0 \\
\hline -Other Inflammatory Conditions & $690-698$ & 0 & 0 & 0 & 0 & 0 & 0 \\
\hline -Other & 700-709 & 0 & 0 & 0 & 0 & 0 & 0 \\
\hline -Arthropathies & $710-719$ & 0 & 0 & 251 & 25 & 57 & 24 \\
\hline -Dorsopathies & $720-724$ & 30 & 0 & 208 & 38 & 5 & 0 \\
\hline -Rheumatism, Excluding Back & 725-729 & 0 & 0 & 0 & 0 & 110 & 0 \\
\hline -Symptoms & $780-789$ & 0 & 0 & 9 & 16 & 0 & 0 \\
\hline -Fracture - Upper Limb & 810-819 & 0 & 0 & 0 & 0 & 0 & 0 \\
\hline -Fracture - Lower Limb & $820-829$ & 0 & 0 & 0 & 0 & 0 & 0 \\
\hline -Dislocation & 830-839 & 0 & 0 & 0 & 0 & 0 & 0 \\
\hline -Sprains \& Strains - Back & 846-847 & 30 & 0 & 192 & 38 & 35 & 0 \\
\hline -Sprains \& Strains - Other & $840-845,848$ & 0 & 0 & 380 & 39 & 57 & 24 \\
\hline -Open Wound - Head, Neck, Trunk & 870-879 & 0 & 0 & 0 & 0 & 0 & 0 \\
\hline -Open Wound - Upper Limb & 880-887 & 0 & 0 & 0 & 0 & 19 & 0 \\
\hline -Open Wound - Lower Limb & $890-897$ & 0 & 0 & 0 & 0 & 0 & 0 \\
\hline -Superficial Injury & $910-919$ & 0 & 0 & 0 & 0 & 0 & 0 \\
\hline -Contusion & $920-924$ & 0 & 0 & 8 & 0 & 0 & 0 \\
\hline -Foreign Body Entering Orifice & 930-939 & 0 & 0 & 0 & 0 & 0 & 0 \\
\hline -Burns & $940-949$ & 0 & 0 & 0 & 0 & 1 & 0 \\
\hline -Complications \& Unspecified Injuries & 958-959 & 0 & 0 & 354 & 22 & 110 & 0 \\
\hline -Toxic Effects - Non-medicinal & 980-989 & 0 & 0 & 0 & 0 & 0 & 0 \\
\hline -Unspecified Effects - External Causes & $990-995$ & 0 & 0 & 0 & 0 & 0 & 0 \\
\hline
\end{tabular}

*OSHA events with >1 ICD-9-CM code in the same diagnostic category were counted only once. Only those diagnostic categories and gender/job category combinations with at least one occurrence appear in this table. 


\section{Lawrence Livermore National Laboratory 2007}

OSHA Data

Appendix U. Number of Occurrences in Each Accident Category by Gender and Job Category*

\begin{tabular}{|c|c|c|c|c|c|c|c|}
\hline & & \multicolumn{6}{|c|}{ Women } \\
\hline & & \multicolumn{5}{|c|}{ Job Category } & \multirow[b]{2}{*}{ TOTAL } \\
\hline & & Professional & $\begin{array}{l}\text { Administrative } \\
\text { Support }\end{array}$ & \begin{tabular}{|c|} 
Technical \\
Support
\end{tabular} & Service & Crafts & \\
\hline Type of Accident & E CODES & \multirow[b]{2}{*}{0} & \multirow[b]{2}{*}{1} & \multirow[b]{2}{*}{0} & \multirow[b]{2}{*}{0} & \multirow[b]{2}{*}{0} & \multirow[b]{2}{*}{1} \\
\hline Motor Vehicle Traffic & E810-E819 & & & & & & \\
\hline Accidental Poisoning - Non-medicinal & E860-E869 & 0 & 0 & 1 & 0 & 0 & 1 \\
\hline Falls & E880-E888 & 1 & 10 & 0 & 0 & 0 & 11 \\
\hline Natural/Environmental Factors & E900-E909 & 0 & 3 & 0 & 0 & 0 & 3 \\
\hline Other Accidents & E916-E928 & 4 & 27 & 5 & 4 & 1 & 41 \\
\hline
\end{tabular}

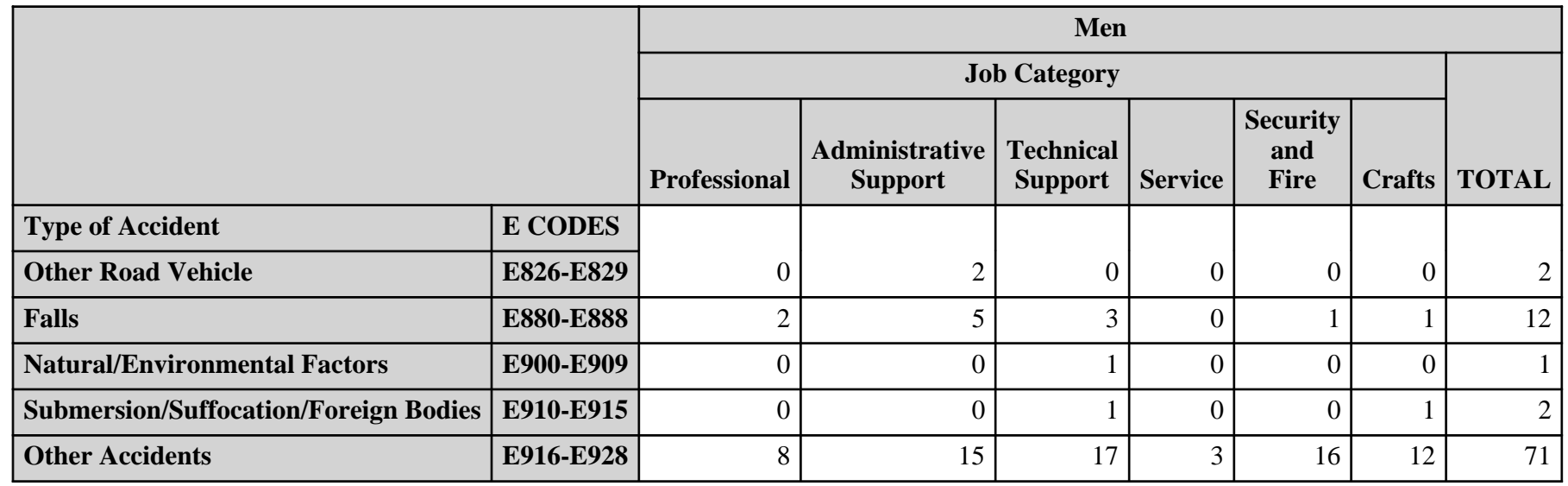

*Only those accident types and gender/job category combinations with at least one occurrence appear in this table. 


\section{Lawrence Livermore National Laboratory 2007}

OSHA Data

Appendix V. Number of Workdays Lost or with Restricted Activity in Each Accident Category by Gender and Job Category*

\begin{tabular}{|c|c|c|c|c|c|c|c|c|c|c|c|}
\hline & & \multicolumn{10}{|c|}{ Women } \\
\hline & & \multicolumn{10}{|c|}{ Job Category } \\
\hline & & \multicolumn{2}{|c|}{ Professional } & \multicolumn{2}{|c|}{$\begin{array}{l}\text { Administrative } \\
\text { Support }\end{array}$} & \multicolumn{2}{|c|}{$\begin{array}{l}\text { Technical } \\
\text { Support }\end{array}$} & \multicolumn{2}{|c|}{ Service } & \multicolumn{2}{|c|}{ Crafts } \\
\hline & & $\begin{array}{c}\text { Days } \\
\text { Restricted }\end{array}$ & $\begin{array}{l}\text { Days } \\
\text { Lost }\end{array}$ & \begin{tabular}{|c|} 
Days \\
Restricted \\
\end{tabular} & $\begin{array}{l}\text { Days } \\
\text { Lost }\end{array}$ & \begin{tabular}{c|} 
Days \\
Restricted
\end{tabular} & $\begin{array}{l}\text { Days } \\
\text { Lost }\end{array}$ & $\begin{array}{c}\text { Days } \\
\text { Restricted }\end{array}$ & $\begin{array}{l}\text { Days } \\
\text { Lost }\end{array}$ & \begin{tabular}{|c|} 
Days \\
Restricted
\end{tabular} & $\begin{array}{l}\text { Days } \\
\text { Lost }\end{array}$ \\
\hline Type of Accident & E Codes & & & & & & & & & & \\
\hline Motor Vehicle Traffic & \begin{tabular}{|l|} 
E810-E819 \\
\end{tabular} & 0 & 0 & 0 & 0 & 0 & 0 & 0 & 0 & 0 & 0 \\
\hline $\begin{array}{l}\text { Accidental } \\
\text { Poisoning - Non-medicinal }\end{array}$ & E860-E869 & 0 & 0 & 0 & 0 & 0 & 0 & 0 & 0 & 0 & 0 \\
\hline Falls & E880-E888 & 0 & 0 & 0 & 208 & 0 & 0 & 0 & 0 & 0 & 0 \\
\hline $\begin{array}{l}\text { Natural/Environmental } \\
\text { Factors }\end{array}$ & E900-E909 & 0 & 0 & 0 & 20 & 0 & 0 & 0 & 0 & 0 & 0 \\
\hline Other Accidents & E916-E928 & 0 & 0 & 124 & 218 & 106 & 207 & 86 & 0 & 138 & 28 \\
\hline
\end{tabular}

\begin{tabular}{|c|c|c|c|c|c|c|c|}
\hline & \multicolumn{6}{|c|}{ Men } \\
\hline & & \multicolumn{6}{|c|}{ Job Category } \\
\hline & & \multicolumn{2}{|c|}{ Professional } & \multicolumn{2}{|c|}{$\begin{array}{l}\text { Administrative } \\
\text { Support }\end{array}$} & \multicolumn{2}{|c|}{$\begin{array}{l}\text { Technical } \\
\text { Support }\end{array}$} \\
\hline & & $\begin{array}{c}\text { Days } \\
\text { Restricted }\end{array}$ & $\begin{array}{l}\text { Days } \\
\text { Lost }\end{array}$ & \begin{tabular}{|c|} 
Days \\
Restricted
\end{tabular} & $\begin{array}{l}\text { Days } \\
\text { Lost }\end{array}$ & $\begin{array}{c}\text { Days } \\
\text { Restricted }\end{array}$ & $\begin{array}{l}\text { Days } \\
\text { Lost }\end{array}$ \\
\hline Type of Accident & E Codes & & & & & & \\
\hline Other Road Vehicle & E826-E829 & 0 & 0 & 0 & 0 & 0 & 0 \\
\hline Falls & E880-E888 & 0 & 0 & 0 & 123 & 0 & 19 \\
\hline Natural/Environmental Factors & E900-E909 & 0 & 0 & 0 & 0 & 0 & 0 \\
\hline Submersion/Suffocation/Foreign Bodies & E910-E915 & 0 & 0 & 0 & 0 & 0 & 0 \\
\hline Other Accidents & E916-E928 & 0 & 0 & 138 & 6 & 163 & 3 \\
\hline
\end{tabular}

\begin{tabular}{|c|c|c|c|c|c|c|c|}
\hline & \multicolumn{6}{|c|}{ Men } \\
\hline & & \multicolumn{6}{|c|}{ Job Category } \\
\hline & & \multicolumn{2}{|c|}{ Service } & \multicolumn{2}{|c|}{$\begin{array}{l}\text { Security and } \\
\text { Fire }\end{array}$} & \multicolumn{2}{|c|}{ Crafts } \\
\hline & & \begin{tabular}{|c|} 
Days \\
Restricted
\end{tabular} & \begin{tabular}{|l|} 
Days \\
Lost
\end{tabular} & $\begin{array}{c}\text { Days } \\
\text { Restricted }\end{array}$ & $\begin{array}{l}\text { Days } \\
\text { Lost }\end{array}$ & $\begin{array}{c}\text { Days } \\
\text { Restricted }\end{array}$ & \begin{tabular}{|l} 
Days \\
Lost
\end{tabular} \\
\hline Type of Accident & E Codes & & & & & & \\
\hline Other Road Vehicle & E826-E829 & 0 & 0 & 0 & 0 & 0 & 0 \\
\hline Falls & E880-E888 & 0 & 0 & 48 & 0 & 57 & 24 \\
\hline Natural/Environmental Factors & E900-E909 & 0 & 0 & 0 & 0 & 0 & $\overline{0}$ \\
\hline Submersion/Suffocation/Foreign Bodies & E910-E915 & 0 & 0 & 0 & 0 & 0 & 0 \\
\hline Other Accidents & E916-E928 & 30 & 0 & 570 & 57 & 165 & 0 \\
\hline
\end{tabular}

*OSHA events with $>1 \mathrm{E}$ code in the same accident type were counted only once. Only those accident types and gender/job category combinations with at least one occurrence appear in this table. 


\section{Lawrence Livermore National Laboratory 2007}

OSHA Data

Appendix W. Age-Adjusted OSHA Illness and Injury Rates by Diagnostic Category*

Part 1. Men

\begin{tabular}{|c|c|c|c|c|c|}
\hline & & $\begin{array}{l}\text { Number of } \\
\text { Diagnoses }\end{array}$ & $\begin{array}{c}\text { Age-Adjusted } \\
\text { Rate per } 1,000 * *\end{array}$ & $\begin{array}{c}\text { Lower } 95 \% \\
\text { Confidence } \\
\text { Limit per 1,000 }\end{array}$ & $\begin{array}{c}\text { Upper } 95 \% \\
\text { Confidence } \\
\text { Limit per 1,000 }\end{array}$ \\
\hline Diagnostic Category & ICD-9-CM Code & & & & \\
\hline INFECTIOUS \& PARASITIC DISEASES (DIS) & 001-139 & 1 & 0.1 & 0.0 & 0.9 \\
\hline -Mycoses & $110-118$ & 1 & 0.1 & 0.0 & 0.9 \\
\hline BLOOD \& BLOOD-FORMING ORGANS & $280-289$ & 1 & 0.1 & 0.0 & 0.7 \\
\hline NERVOUS SYSTEM (NS) \& SENSE ORGANS & $320-389$ & 4 & 0.5 & 0.2 & 1.3 \\
\hline -Disorders of Peripheral NS & $350-359$ & 2 & 0.2 & 0.1 & 1.0 \\
\hline -Dis of Ear \& Mastoid & $380-389$ & 2 & 0.2 & 0.1 & 1.0 \\
\hline RESPIRATORY SYSTEM & $460-519$ & 1 & 0.1 & 0.0 & 0.7 \\
\hline -Lung Dis from External Agents & $500-508$ & 1 & 0.1 & 0.0 & 0.7 \\
\hline DIGESTIVE SYSTEM & $520-579$ & 1 & 0.1 & 0.0 & 0.7 \\
\hline -Hernia & $550-553$ & 1 & 0.1 & 0.0 & 0.7 \\
\hline SKIN \& SUBCUTANEOUS TISSUE & $680-709$ & 2 & 0.6 & 0.1 & 2.2 \\
\hline -Other Inflammatory Conditions & $690-698$ & 1 & 0.3 & 0.0 & 2.0 \\
\hline -Other & 700-709 & 1 & 0.3 & 0.0 & 2.0 \\
\hline $\begin{array}{l}\text { MUSCULOSKELETAL \& CONNECTIVE } \\
\text { TISSUE }\end{array}$ & 710-739 & 64 & 11.4 & 8.6 & 15.1 \\
\hline -Arthropathies & 710-719 & 39 & 6.9 & 4.8 & 9.8 \\
\hline -Dorsopathies & $720-724$ & 16 & 2.6 & 1.5 & 4.4 \\
\hline -Rheumatism, Excluding Back & 725-729 & 9 & 1.9 & 0.9 & 4.3 \\
\hline $\begin{array}{l}\text { SYMPTOMS, SIGNS, \& ILL-DEFINED } \\
\text { CONDITIONS }\end{array}$ & 780-799 & 13 & 2.5 & 1.3 & 4.6 \\
\hline -Symptoms & 780-789 & 13 & 2.5 & 1.3 & 4.6 \\
\hline INJURY \& POISONING & $800-999$ & 92 & 17.1 & 13.5 & 21.7 \\
\hline -Fracture - Upper Limb & \begin{tabular}{|l|}
$810-819$ \\
\end{tabular} & 3 & 0.5 & 0.1 & 1.7 \\
\hline -Fracture - Lower Limb & $820-829$ & 1 & 0.1 & 0.0 & 0.7 \\
\hline -Dislocation & $830-839$ & 3 & 0.7 & 0.2 & 2.2 \\
\hline -Sprains \& Strains - Back & 846-847 & 12 & 1.6 & 0.9 & 2.9 \\
\hline -Sprains \& Strains - Other & $840-845,848$ & 28 & 4.6 & 3.0 & 7.1 \\
\hline -Open Wound - Head, Neck, Trunk & 870-879 & 2 & 0.7 & 0.2 & 3.0 \\
\hline -Open Wound - Upper Limb & $880-887$ & 9 & 1.8 & 0.9 & 3.9 \\
\hline -Open Wound - Lower Limb & 890-897 & 1 & 0.1 & 0.0 & 0.9 \\
\hline -Superficial Injury & $910-919$ & 1 & 0.4 & 0.1 & 3.2 \\
\hline -Contusion & $920-924$ & 8 & 1.8 & 0.8 & 4.0 \\
\hline -Foreign Body Entering Orifice & 930-939 & 2 & 0.2 & 0.1 & 0.9 \\
\hline -Burns & $940-949$ & 1 & 0.1 & 0.0 & 0.9 \\
\hline -Toxic Effects - Non-medicinal & $980-989$ & 2 & 0.4 & 0.1 & 1.8 \\
\hline -Complications of Surgical/Medical Care & 996-999 & 19 & 4.0 & 2.4 & 6.7 \\
\hline Total & & 179 & 32.5 & 27.4 & 38.4 \\
\hline
\end{tabular}

* Only those diagnostic categories with at least one occurrence appear in this table.

**Standardized to age distribution of 2000 U.S. population. 
Lawrence Livermore National Laboratory 2007

OSHA Data

Appendix W. Age-Adjusted OSHA Illness and Injury Rates by Diagnostic Category*

Part 2. Women

\begin{tabular}{|c|c|c|c|c|c|}
\hline & & $\begin{array}{l}\text { Number of } \\
\text { Diagnoses }\end{array}$ & $\begin{array}{c}\text { Age-Adjusted } \\
\text { Rate per } 1,000 * *\end{array}$ & $\begin{array}{c}\text { Lower } 95 \% \\
\text { Confidence } \\
\text { Limit per 1,000 }\end{array}$ & $\begin{array}{c}\text { Upper } 95 \% \\
\text { Confidence } \\
\text { Limit per 1,000 }\end{array}$ \\
\hline Diagnostic Category & ICD-9-CM Code & & & & \\
\hline INFECTIOUS \& PARASITIC DISEASES (DIS) & 001-139 & 1 & 0.2 & 0.0 & 1.7 \\
\hline -Mycoses & $110-118$ & 1 & 0.2 & 0.0 & 1.7 \\
\hline NERVOUS SYSTEM (NS) \& SENSE ORGANS & 320-389 & 4 & 1.8 & 0.6 & 5.5 \\
\hline -Disorders of Peripheral NS & $350-359$ & 4 & 1.8 & 0.6 & 5.5 \\
\hline RESPIRATORY SYSTEM & $460-519$ & 1 & 0.8 & 0.1 & 5.6 \\
\hline -Lung Dis from External Agents & $500-508$ & 1 & 0.8 & 0.1 & $\overline{5.6}$ \\
\hline DIGESTIVE SYSTEM & \begin{tabular}{|c|}
$520-579$ \\
\end{tabular} & 1 & 0.5 & 0.1 & 3.9 \\
\hline -Hernia & $550-553$ & 1 & 0.5 & 0.1 & 3.9 \\
\hline SKIN \& SUBCUTANEOUS TISSUE & $680-709$ & 2 & 1.4 & 0.3 & 5.6 \\
\hline -Infections & $680-686$ & 1 & 0.6 & 0.1 & 4.1 \\
\hline -Other Inflammatory Conditions & $690-698$ & 1 & 0.8 & 0.1 & 5.6 \\
\hline $\begin{array}{l}\text { MUSCULOSKELETAL \& CONNECTIVE } \\
\text { TISSUE }\end{array}$ & \begin{tabular}{|c|}
$710-739$ \\
\end{tabular} & 41 & 13.9 & 9.8 & 19.7 \\
\hline -Arthropathies & $710-719$ & 30 & 9.6 & 6.4 & 14.3 \\
\hline -Dorsopathies & $720-724$ & 4 & 1.6 & 0.6 & 4.6 \\
\hline -Rheumatism, Excluding Back & $725-729$ & 7 & 2.7 & 1.1 & 6.6 \\
\hline $\begin{array}{l}\text { SYMPTOMS, SIGNS, \& ILL-DEFINED } \\
\text { CONDITIONS }\end{array}$ & $780-799$ & 9 & 3.6 & 1.8 & 7.5 \\
\hline -Symptoms & $780-789$ & 8 & 3.4 & 1.6 & 7.3 \\
\hline -Non-Specific Abnormal Findings & $790-796$ & 1 & 0.2 & 0.0 & 1.7 \\
\hline INJURY \& POISONING & \begin{tabular}{|l|}
$800-999$ \\
\end{tabular} & 54 & 19.2 & 14.3 & 25.9 \\
\hline -Fracture - Upper Limb & $810-819$ & 1 & 0.2 & 0.0 & 1.6 \\
\hline -Sprains \& Strains - Back & 846-847 & 4 & 1.6 & 0.6 & 4.7 \\
\hline -Sprains \& Strains - Other & $840-845,848$ & 15 & 4.1 & 2.4 & 7.2 \\
\hline -Superficial Injury & $910-919$ & 2 & 0.8 & 0.2 & 3.6 \\
\hline -Contusion & $920-924$ & 5 & 2.1 & 0.8 & 5.6 \\
\hline -Toxic Effects - Non-medicinal & $980-989$ & 2 & 0.5 & 0.1 & 1.9 \\
\hline -Complications of Surgical/Medical Care & 996-999 & 25 & 9.9 & 6.4 & 15.3 \\
\hline Total & & 113 & 41.6 & 33.8 & 51.2 \\
\hline
\end{tabular}

* Only those diagnostic categories with at least one occurrence appear in this table.

$* *$ Standardized to age distribution of 2000 U.S. population. 


\section{Lawrence Livermore National Laboratory 2007}

OSHA Data

Appendix W. Age-Adjusted OSHA Illness and Injury Rates by Diagnostic Category*

Part 3. Men and Women

\begin{tabular}{|c|c|c|c|c|c|}
\hline & & $\begin{array}{l}\text { Number of } \\
\text { Diagnoses }\end{array}$ & $\begin{array}{c}\text { Age-Adjusted } \\
\text { Rate per } 1,000 * *\end{array}$ & $\begin{array}{c}\text { Lower } 95 \% \\
\text { Confidence } \\
\text { Limit per } 1,000\end{array}$ & $\begin{array}{c}\text { Upper } 95 \% \\
\text { Confidence } \\
\text { Limit per 1,000 }\end{array}$ \\
\hline Diagnostic Category & ICD-9-CM Code & \multirow[b]{2}{*}{2} & \multirow[b]{2}{*}{0.2} & \multirow[b]{2}{*}{0.0} & \multirow[b]{2}{*}{0.7} \\
\hline INFECTIOUS \& PARASITIC DISEASES (DIS) & 001-139 & & & & \\
\hline -Mycoses & $110-118$ & 2 & 0.2 & 0.0 & 0.7 \\
\hline BLOOD \& BLOOD-FORMING ORGANS & $280-289$ & 1 & 0.1 & 0.0 & 0.5 \\
\hline NERVOUS SYSTEM (NS) \& SENSE ORGANS & 320-389 & 8 & 1.0 & 0.4 & 2.1 \\
\hline -Disorders of Peripheral NS & $350-359$ & 6 & 0.8 & 0.3 & 2.0 \\
\hline -Dis of Ear \& Mastoid & $380-389$ & 2 & 0.2 & 0.0 & 0.7 \\
\hline RESPIRATORY SYSTEM & $460-519$ & 2 & 0.4 & 0.1 & 1.8 \\
\hline -Lung Dis from External Agents & $500-508$ & 2 & 0.4 & 0.1 & 1.8 \\
\hline DIGESTIVE SYSTEM & $520-579$ & 2 & 0.2 & 0.0 & 0.7 \\
\hline -Hernia & $550-553$ & 2 & 0.2 & 0.0 & 0.7 \\
\hline SKIN \& SUBCUTANEOUS TISSUE & $680-709$ & 4 & 0.8 & 0.3 & 2.3 \\
\hline -Infections & $680-686$ & 1 & 0.2 & 0.0 & 1.3 \\
\hline -Other Inflammatory Conditions & $690-698$ & 2 & 0.5 & 0.1 & 1.9 \\
\hline -Other & 700-709 & 1 & 0.2 & 0.0 & 1.3 \\
\hline $\begin{array}{l}\text { MUSCULOSKELETAL \& CONNECTIVE } \\
\text { TISSUE }\end{array}$ & $710-739$ & 105 & 12.2 & 9.8 & 15.2 \\
\hline -Arthropathies & $710-719$ & 69 & 7.8 & 6.0 & 10.1 \\
\hline -Dorsopathies & $720-724$ & 20 & 2.2 & 1.4 & 3.6 \\
\hline -Rheumatism, Excluding Back & $725-729$ & 16 & 2.2 & 1.2 & 4.0 \\
\hline $\begin{array}{l}\text { SYMPTOMS, SIGNS, \& ILL-DEFINED } \\
\text { CONDITIONS }\end{array}$ & $780-799$ & 22 & 2.7 & 1.7 & 4.4 \\
\hline -Symptoms & $780-789$ & 21 & 2.6 & 1.6 & 4.3 \\
\hline -Non-Specific Abnormal Findings & $790-796$ & 1 & 0.1 & 0.0 & 0.6 \\
\hline INJURY \& POISONING & $800-999$ & 146 & 17.2 & 14.3 & 20.8 \\
\hline -Fracture - Upper Limb & 810-819 & 4 & 0.4 & 0.1 & 1.2 \\
\hline -Fracture - Lower Limb & $820-829$ & 1 & 0.1 & 0.0 & 0.5 \\
\hline -Dislocation & $830-839$ & 3 & 0.5 & 0.1 & 1.5 \\
\hline -Sprains \& Strains - Back & $846-847$ & 16 & 1.6 & 0.9 & 2.7 \\
\hline -Sprains \& Strains - Other & $840-845,848$ & 43 & 4.3 & 3.1 & 6.1 \\
\hline -Open Wound - Head, Neck, Trunk & 870-879 & 2 & 0.5 & 0.1 & 1.9 \\
\hline -Open Wound - Upper Limb & 880-887 & 9 & 1.2 & 0.6 & 2.6 \\
\hline -Open Wound - Lower Limb & $890-897$ & 1 & 0.1 & 0.0 & 0.6 \\
\hline -Superficial Injury & $910-919$ & 3 & 0.5 & 0.2 & 1.9 \\
\hline -Contusion & $920-924$ & 13 & 1.9 & 1.0 & 3.6 \\
\hline -Foreign Body Entering Orifice & 930-939 & 2 & 0.1 & 0.0 & 0.6 \\
\hline -Burns & $940-949$ & 1 & 0.1 & 0.0 & 0.7 \\
\hline -Toxic Effects - Non-medicinal & $980-989$ & 4 & 0.4 & 0.1 & 1.2 \\
\hline
\end{tabular}

*Only those diagnostic categories with at least one occurrence appear in this table.

$* *$ Standardized to age distribution of 2000 U.S. population. 
Lawrence Livermore National Laboratory 2007

OSHA Data

Appendix W. Age-Adjusted OSHA Illness and Injury Rates by Diagnostic Category*

\begin{tabular}{|c|c|c|c|c|c|}
\hline & & $\begin{array}{c}\text { Number of } \\
\text { Diagnoses }\end{array}$ & $\begin{array}{c}\text { Age-Adjusted } \\
\text { Rate per 1,000** }\end{array}$ & $\begin{array}{c}\text { Lower } 95 \% \\
\text { Confidence } \\
\text { Limit per } 1,000\end{array}$ & $\begin{array}{c}\text { Upper } 95 \% \\
\text { Confidence } \\
\text { Limit per 1,000 }\end{array}$ \\
\hline Diagnostic Category & ICD-9-CM Code & \multirow[b]{2}{*}{44} & \multirow[b]{2}{*}{5.5} & \multirow[b]{2}{*}{3.9} & \multirow[b]{2}{*}{7.7} \\
\hline -Complications of Surgical/Medical Care & 996-999 & & & & \\
\hline Total & & 292 & 34.7 & 30.4 & 39.6 \\
\hline
\end{tabular}

* Only those diagnostic categories with at least one occurrence appear in this table.

**Standardized to age distribution of 2000 U.S. population. 